\title{
DEVELOPMENT AND VALIDATION OF A TIBIOFEMORAL JOINT FINITE ELEMENT MODEL AND SUBSEQUENT GAIT ANALYSIS OF INTACT ACL AND ACL DEFICIENT INDIVIDUALS
}

\author{
A Thesis \\ presented to \\ the Faculty of California Polytechnic State University, \\ San Luis Obispo \\ In Partial Fulfillment \\ of the Requirements for the Degree \\ Master of Science in Mechanical Engineering \\ by \\ Nicholas Czapla
}

June 2015 
(C) 2015

Nicholas Czapla

\section{ALL RIGHTS RESERVED}


TITLE:

AUTHOR:

DATE SUBMITTED:

COMMITTEE MEMBER:
COMMITTEE CHAIR:

Development and Validation of a Tibiofemoral Joint Finite Element Model and Subsequent Gait Analysis of Intact ACL and ACL Deficient Individuals

Nicholas Czapla

June 2015

Stephen Klisch, Ph.D.

Professor of Mechanical Engineering

Scott Hazelwood, Ph.D.

Professor of Biomedical Engineering

COMMITTEE MEMBER: Joseph Mello, Ph.D.

Professor of Mechanical Engineering 


\section{ABSTRACT}

Development and Validation of a Tibiofemoral Joint Finite Element Model and Subsequent Gait Analysis of Intact ACL and ACL Deficient Individuals

\section{Nicholas Czapla}

Osteoarthritis $(\mathrm{OA})$ is a degenerative condition of articular cartilage that affects more than 25 million people in the US. Joint injuries, like anterior cruciate ligament $(A C L)$ tears, can lead to $O A$ due to a change in articular cartilage loading. Gait analysis combined with knee joint finite element modeling (FEM) has been used to predict the articular cartilage loading. To predict the change of articular cartilage loading during gait due to various $A C L$ injuries, a tibiofemoral FEM was developed from magnetic resonance images (MRIs) of a 33 year male, with no prior history of knee injuries. The FEM was validated for maximum contact pressure and anterior tibial translation using cadaver knee studies. The FEM was used to model gait of knees with an intact $A C L$, anteromedial (AM) bundle injury, posterolateral (PL) bundle injury, complete $A C L$ injury, $A M$ deficiency, PL deficiency, complete ACL rupture, as well as a bone-patellar tendon-bone (BPTB) graft. Generally, the predicted maximum contact pressure and contact area increased for all the $\mathrm{ACL}$ injuries when compared to intact ACLs. While an increase in maximum contact pressure and contact area is an indication of an increased risk of the development of $O A$, the percent of increase was typically small suggesting that walking is a safe activity for individuals with ACL injuries. 
Keywords: Osteoarthritis, finite element, gait analysis, articular cartilage, anterior cruciate ligament, human knee-joint 


\section{ACKNOWLEDGMENTS}

Thanks to Dr. Stephen Klisch and Dr. Scott Hazelwood for their mentorship and guidance in the completion of this thesis. Also, thanks to Meghan Sylvia for her assistance, and friendship.

A special thanks to Dr. Saikat Pal, Biomechanical Department, for providing the MRIs.

A special thanks to Dr. David Tuttle and Dr. Otto Schueckler for their assistance in reading MRIs and planning the study.

A special thanks to Zach Lerner, CSU Fort Collins Physical Activity Energetic and Mechanics Laboratory (PAL), for the necessary data to perform an accurate finite element gait analysis.

Lastly, thank you to my family and friends for their constant love and support. 


\section{TABLE OF CONTENTS}

Page

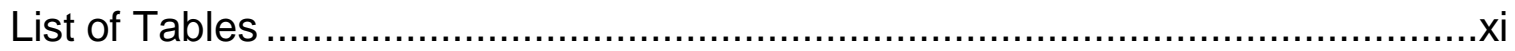

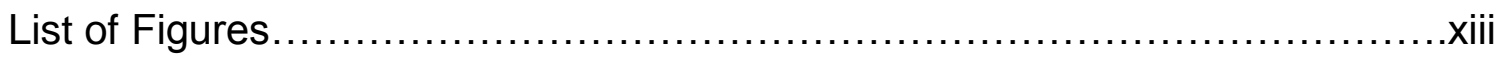

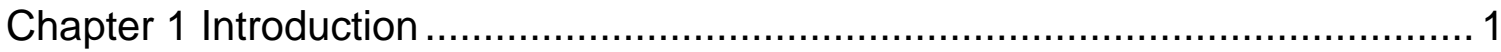

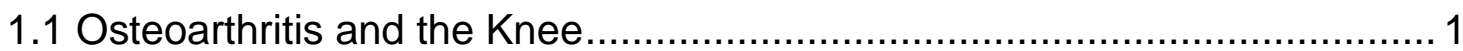

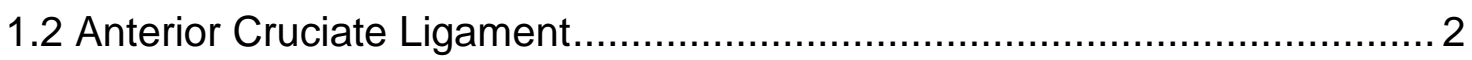

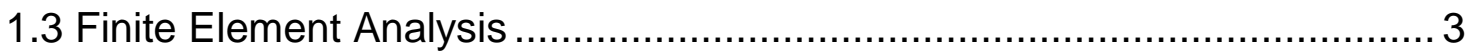

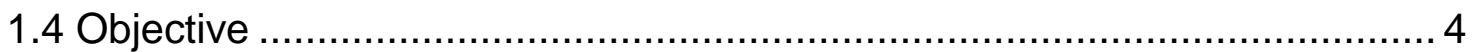

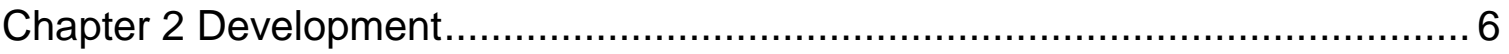

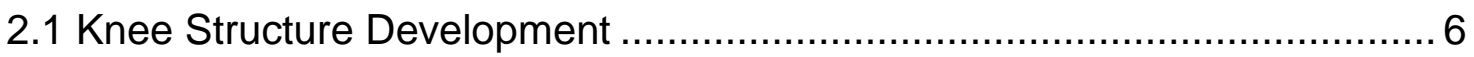

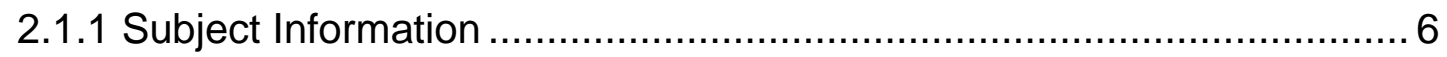

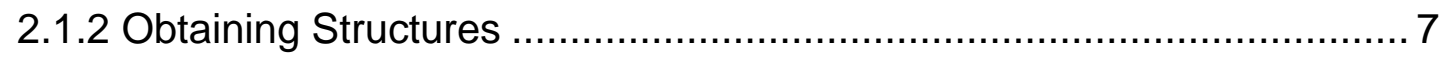

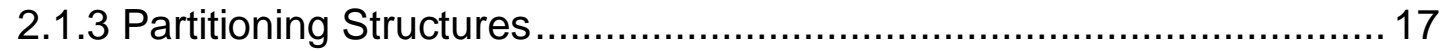

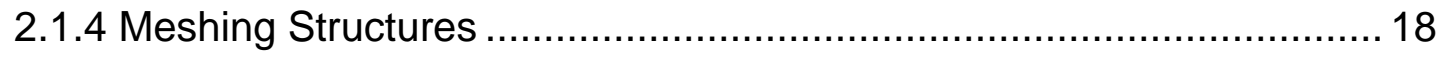

2.2 Finite Element Model Development..................................................... 24

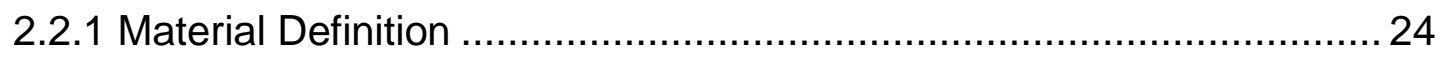

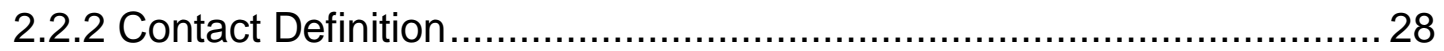

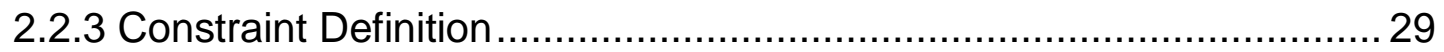

2.2.4 Meniscal Attachment Constraints.................................................... 30 
2.2.5 Lateral Collateral Ligament Distal Face Constraints 32

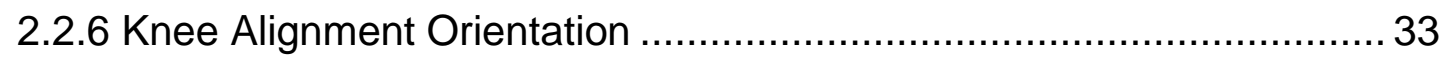

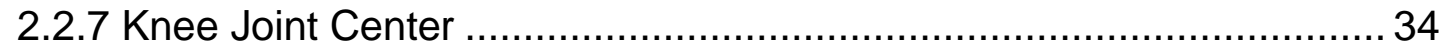

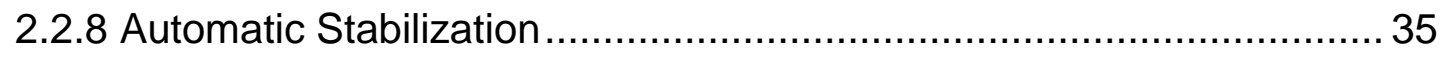

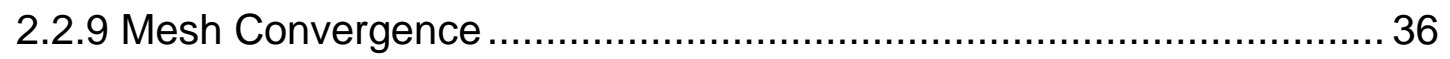

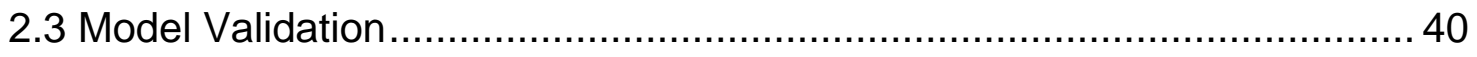

2.3.1 Maximum Tibiofemoral Contact Pressure ...................................... 41

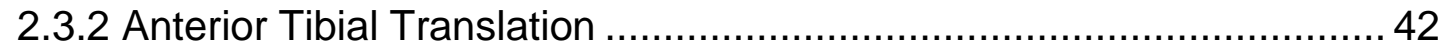

2.3.3 Anteromedial Bundle Strain ........................................................... 43

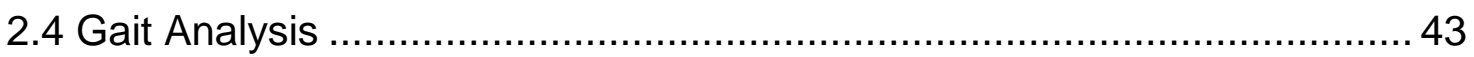

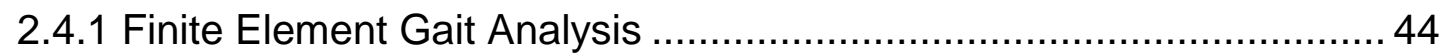

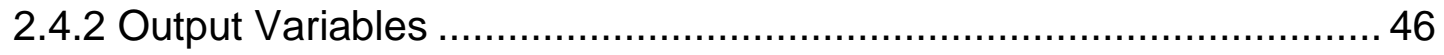

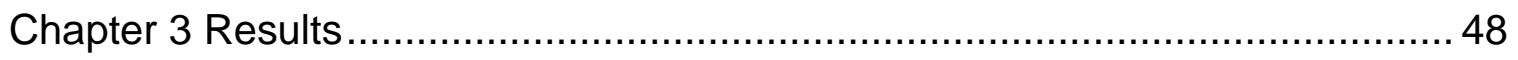

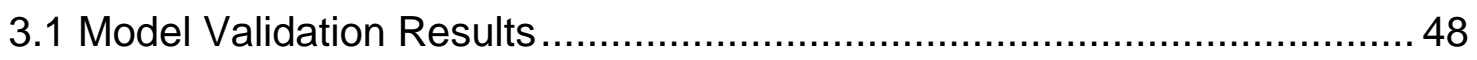

3.1.1 Maximum Tibiofemoral Contact Pressure ….................................... 48

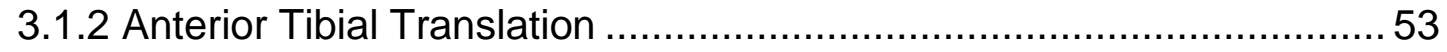

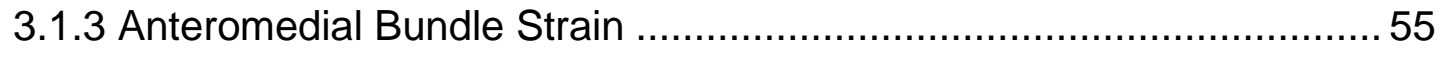

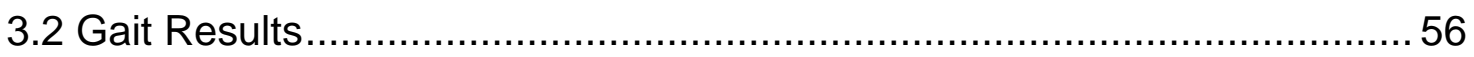

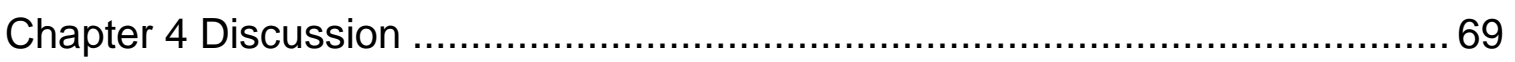

4.1 Tibiofemoral Contact Pressure and Contact Area .................................... 69 
4.1.1 Changes to Cartilage Loading with respect to Anterior Cruciate Ligament Deficiency and Reconstruction ............................................... 69

4.1.2 Changes to Cartilage Loading Throughout Gait ............................... 70

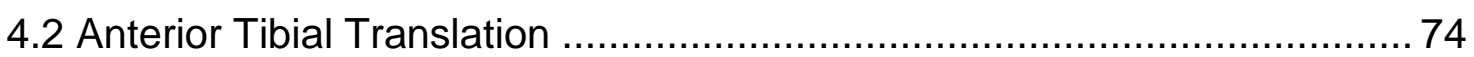

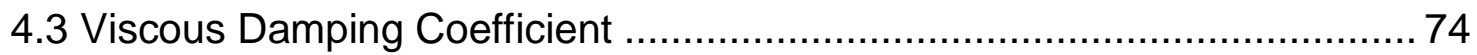

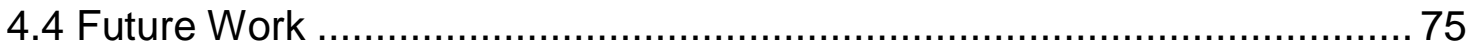

4.4.1 Anterior Cruciate Ligament Deficient Gait Loading …....................... 75

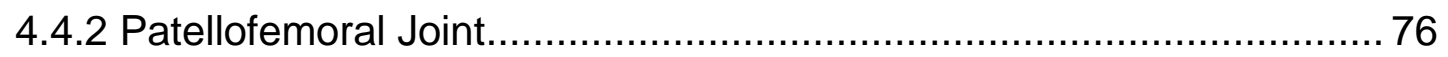

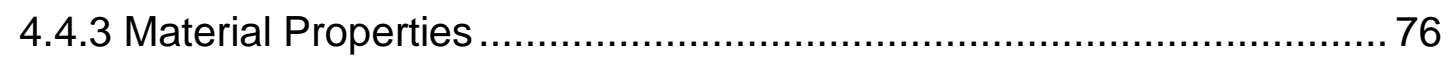

4.4.4 Automatic Stabilization Constant Damping Factor .............................. 78

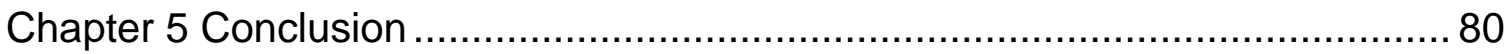

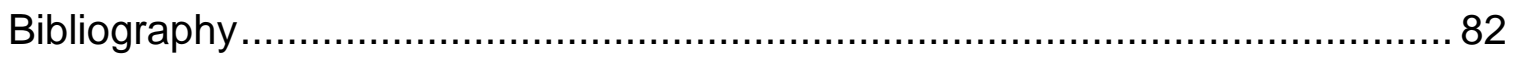

Appendices

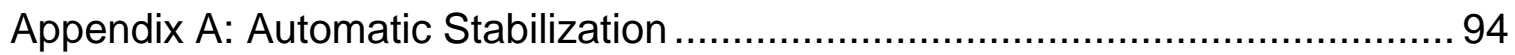

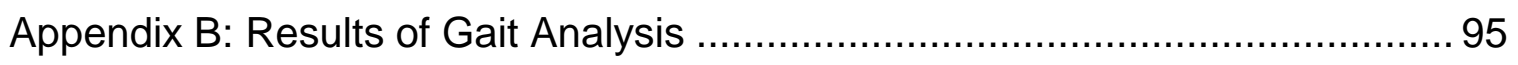

Appendix C: Changes to $\mathrm{CP}$ and $\mathrm{CA}$ for $\mathrm{AM}$ deficient, $\mathrm{PL}$ deficient, $\mathrm{ACL}$

ruptured and ACL RS knees throughout gait ............................................... 115

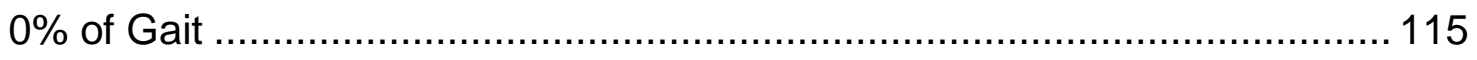

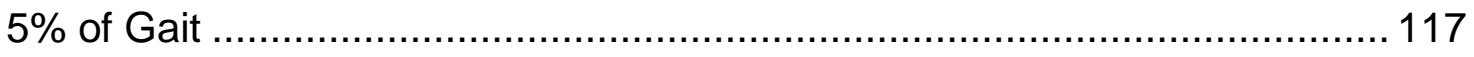

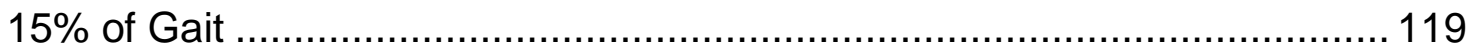


$30 \%$ of Gait

$46 \%$ of Gait 123

$60 \%$ of Gait 125 


\section{LIST OF TABLES}

Table

Page

Table 1: Subject Information. 6

Table 2: Anatomical description of AM and PL bundles [54-57].................... 12

Table 3: Anatomical description of the BPTB graft $[54,56,58,59] \ldots \ldots \ldots \ldots \ldots \ldots 13$

Table 4: Anatomical description of the LCL [60-65] ...................................... 14

Table 5: Anatomical description of the MCL [60-65] ..................................... 15

Table 6: Ligament Properties [72, 79-85] ............................................... 28

Table 7: Meniscal Attachment Horn Stiffness [34]........................................ 31

Table 8: LCL Longitudinal and Transverse Spring Stiffness $[75,76] \ldots \ldots \ldots \ldots \ldots . . . .33$

Table 9: Knee Joint Forces, Moments and Flexion Angles used in the gait

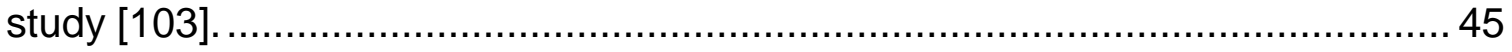

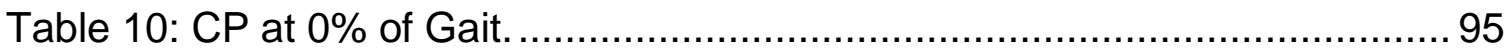

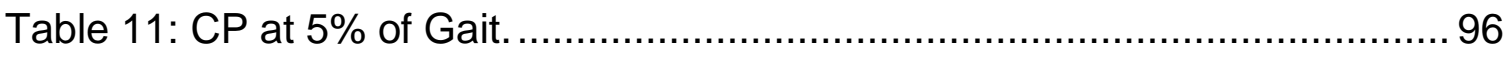

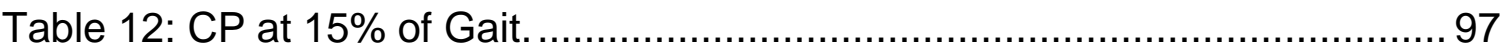

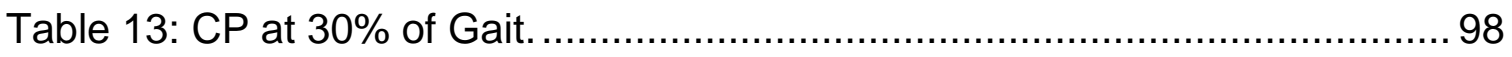

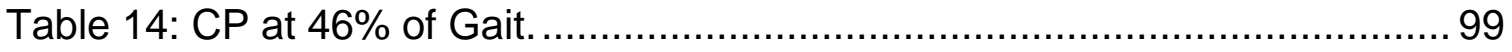

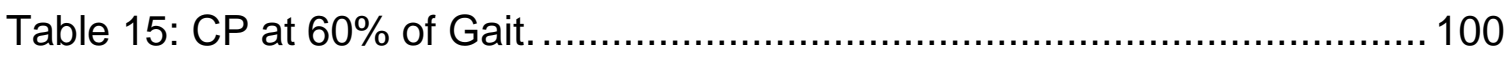

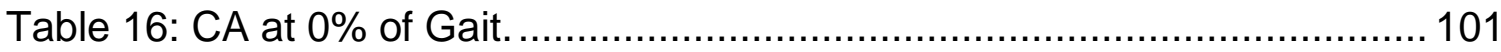

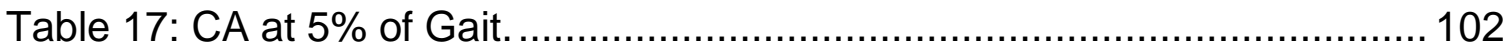

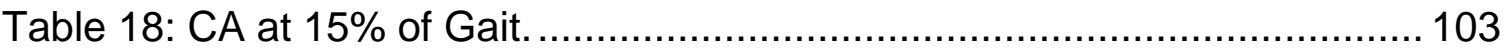

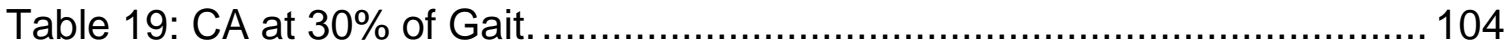

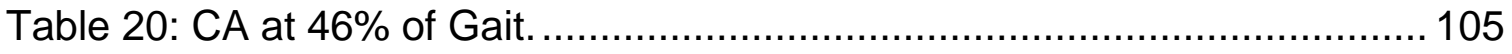




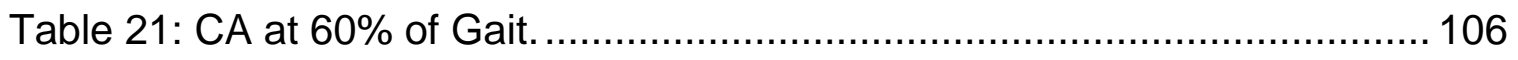

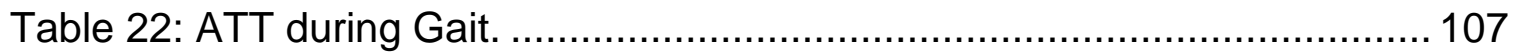

Table 23: Viscous Forces to Total Forces Ratio........................................... 108 


\section{LIST OF FIGURES}

Figure

Page

Figure 1: MR image with A) No mask. B) ACL mask. C) Resulting 3-D object of the ACL

Figure 2: Lateral Tibial Cartilage: A) Surface Mesh Imported from Mimics.

B) Selected Curved Edges to be deleted. C) Smoothed Surface Edges after Curved Edges have been deleted. D) Flat Surface Lofted connecting the Smoothed Surface Edges 9

Figure 3: PCL Before (A) and After (B) sharp points were smoothed. 10

Figure 4: Example of slight overlap between tibia and lateral tibial cartilage. 11

Figure 5: Anterior and Posterior view of assembled knee joint. Ligaments are yellow, bone is grey, articular cartilage is cyan, menisci are purple, AM bundle is blue, PL bundle is red, BPTB graft is green.

Figure 6: A) Femur with partitioning lines used for meshing. B) PCL with partitioning lines used for meshing.

Figure 7: A) Surfaces and curves of the tibia created from "iges" command.

B) Trimmed 2-D array of computational blocks created with "block" command.

C) Unrefined projection of computational blocks onto tibia geometry.

D) Refined projection of computational blocks onto tibia geometry.

Figure 8: A) Surfaces and curves of the PL bundle created from "iges"

command. B) Trimmed 3-D array of computational blocks created with "block" command. C) Unrefined projection of computational blocks onto PL geometry.

D) Refined projection of computational blocks onto PL geometry. 
Figure 9: Anterior and Posterior view of meshed knee joint. Ligaments are yellow, bone is grey, articular cartilage is cyan, menisci are purple, AM bundle is blue, PL bundle is red, BPTB graft is green.

Figure 10: Meniscal Attachment Springs. Medial Anterior Horn is light green, Medial Posterior Horn is dark green, Lateral Anterior Horn is red, and Lateral Posterior Horn is yellow.

Figure 11: Location of KJC shown in red. 35

Figure 12: Mesh Nodes of the femoral cartilage (FC), medial tibial cartilage (MTC), lateral tibial cartilage (LTC), PL, AM and BPTB meshed structures used in the Mesh Convergence Study.

Figure 13: Sample of Convergence study results of the AM bundle, PL bundle, Femoral Cartilage (FC), and Medial tibial Cartilage (MTC)

Figure 14: Contact Pressure of the medial and lateral tibial cartilage during loading at full extension with an intact ACL.

Figure 15: FEA Validation vs Experimental Results of CP during loading with an intact ACL. A) $500 \mathrm{~N}$ Load. B) $1000 \mathrm{~N}$ Load [41]. 50

Figure 16: Medial and lateral tibial cartilage contact pressure during loading at $15^{\circ}$ and $30^{\circ}$ with an intact $A C L$

Figure 17: FEA Validation vs. Experimental Results of CP during loading with an intact ACL. A Loading at $15^{\circ}$ Flexion. B Loading at $30^{\circ}$ Flexion [42].

Figure 18: Morimoto, Seitz and FEA results with $1000 \mathrm{~N}$ compressive load at $A 0^{\circ}$ and $B 30^{\circ}[41,42]$ with intact $A C L$.

Figure 19: FEA Validation vs Experimental Results of ATT [27]. 
Figure 20: FEA Validation vs. Experimental Results of AM Bundle Strain under $140 \mathrm{~N}$ tibial anterior load [94] 56

Figure 21: The point of Gait where the largest percent increase of CP occurred for each articular cartilage between intact $A C L$ and $A M$ deficient Knees. 58 Figure 22: Contour CP plot of each articular cartilage at the point of Gait when there was the largest percent increase of maximum increase of CP between intact ACL and AM deficient Knees.

Figure 23: The point of Gait where the largest percent increase of CP occurred for each articular cartilage between intact $A C L$ and $P L$ deficient Knees.

Figure 24: Contour CP plot of each articular cartilage at the point of Gait when there was the largest percent increase of maximum increase of CP between intact ACL and AM deficient Knees.

Figure 25: The point of Gait where the largest percent increase of CP occurred for each articular cartilage between intact $A C L$ and $A C L$ ruptured Knees. 62 Figure 26: Contour CP plot of each articular cartilage at the point of Gait when there was the largest percent increase of maximum increase of $\mathrm{CP}$ between intact $A C L$ and $A C L$ ruptured Knees. Figure 27: The point of Gait where the largest percent increase of CP occurred for each articular cartilage between intact ACL and ACL RS Knees. 64 Figure 28: Contour CP plot of each articular cartilage at the point of Gait when there was the largest percent increase of maximum increase of CP between intact ACL and ACL RS Knees. 
Figure 29: The point of Gait where the largest percent increase of CA occurred for each articular cartilage between intact $\mathrm{ACL}$ and $\mathrm{AM}$ deficient

Knees. 66

Figure 30: The point of Gait where the largest percent increase of CA occurred for each articular cartilage between intact $A C L$ and $P L$ deficient Knees. The CA of the lateral tibial cartilage decreased at every point of Gait for all various types of $A C L$ injuries.

Figure 31: The point of Gait where the largest percent increase of CA occurred for each articular cartilage between intact ACL and ACL ruptured Knees. 67

Figure 32: The point of Gait where the largest percent increase of CP occurred for each articular cartilage between intact ACL and ACL RS Knees... 68 Figure 33: Articular Cartilage Contact Pressure at 0\% of Gait........................ 109 Figure 34: Articular Cartilage Contact Pressure at 5\% of Gait........................ 110

Figure 35: Articular Cartilage Contact Pressure at 15\% of Gait...................... 111

Figure 36: Articular Cartilage Contact Pressure at 30\% of Gait....................... 112

Figure 37: Articular Cartilage Contact Pressure at 46\% of Gait...................... 113

Figure 38: Articular Cartilage Contact Pressure at 60\% of Gait...................... 114

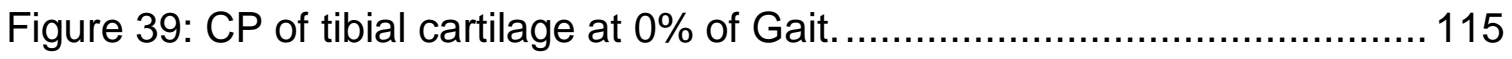

Figure 40: CP of femoral cartilage at 0\% of Gait. ...................................... 115

Figure 41: CA of tibial cartilage at $0 \%$ of Gait................................................ 116

Figure 42: CA of femoral cartilage at $0 \%$ of Gait. ........................................ 116

Figure 43: CP of tibial cartilage at 5\% of Gait........................................... 117 
Figure 44: CP of femoral cartilage at 5\% of Gait. ..................................... 117

Figure 45: CA of tibial cartilage at 5\% of Gait............................................ 118

Figure 46: CA of femoral cartilage at 5\% of Gait. ...................................... 118

Figure 47: CP of tibial cartilage at 15\% of Gait........................................... 119

Figure 48: CP of femoral cartilage at 15\% of Gait. ....................................... 119

Figure 49: CA of tibial cartilage at 15\% of Gait.......................................... 120

Figure 50: CA of femoral cartilage at 15\% of Gait. ....................................... 120

Figure 51: CP of tibial cartilage at 30\% of Gait........................................ 121

Figure 52: CP of femoral cartilage at 30\% of Gait. ..................................... 121

Figure 53: CA of tibial cartilage at 30\% of Gait.......................................... 122

Figure 54: CA of femoral cartilage at 30\% of Gait. ..................................... 122

Figure 55: CP of tibial cartilage at 46\% of Gait......................................... 123

Figure 56: CP of femoral cartilage at $46 \%$ of Gait. ........................................ 123

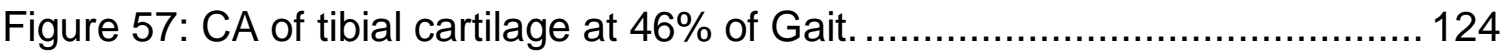

Figure 58: CA of femoral cartilage at 46\% of Gait. ..................................... 124

Figure 59: CP of tibial cartilage at 60\% of Gait............................................ 125

Figure 60: CP of femoral cartilage at $60 \%$ of Gait. ......................................... 125

Figure 61 : CA of tibial cartilage at $60 \%$ of Gait........................................... 126

Figure 62: CA of femoral cartilage at 60\% of Gait. ....................................... 126 


\section{CHAPTER 1 INTRODUCTION}

\subsection{Osteoarthritis and the Knee}

Osteoarthritis $(O A)$ is one of the principal causes of chronic disability in the elderly and reportedly affected 26.9 million individuals living in the US in 2005 [1]. If current trends hold the number will only worsen, leading to an expected 67 million adults living in the US diagnosed with arthritis by 2030 [2]. The financial cost of OA on the US health care system is staggering; total health care costs were $\$ 185$ billion in 2007 , averaging $\$ 2600$ per person [1], [3]. It affects more than just an individual's physical health: $18 \%$ of people with arthritis reported having major depression as well; 1 in 3 people suffering from arthritis reported arthritis-attributed work limitations; adults with arthritis reported having two to four times as many unhealthy days in the past month compared to those without arthritis; 21 million people with arthritis reported a significant limitation in daily activities, such as walking a quarter mile, climbing stairs or bending over [4].

$\mathrm{OA}$ is a joint disorder indicated by the deterioration and loss of the articular cartilage in joints over time. OA commonly affects the ankles, feet, hands, spine, neck and most predominantly the knees and hips [1], [5]. While it is not known exactly what causes $\mathrm{OA}$, there are established indicators linked to a higher likelihood of developing OA such as genetic predisposition, gender, occupation, joint alignment and joint injury [6], [7]. At this time, there is no cure of $\mathrm{OA}$ and typical pharmaceutical remedies include analgesics and antiinflammatories [8]. Total joint replacement is still the standard surgery for 
advanced, debilitating OA, reportedly costing a total of $\$ 32$ billion in the US for knee and hip replacements in 2009 [1], [9].

A considerable amount of work has gone into studying the underlying causes, mechanisms and possible deterrents of the onset of OA, especially in the knee [10-14]. A previous study showed that men with a history of knee injury were five times more at risk for developing OA [6]. Cruciate ligament injuries can lead to OA because the tear is associated with an increased articular cartilage contact stress due to the knee's inability to adequately distribute loads [15]. Anterior cruciate ligament $(\mathrm{ACL})$ tears are among the most common knee injury among the general population and athletes, affecting roughly 200,000 people every year [16], [17]. They are especially common among athletes participating in soccer, football, basketball and alpine skiing [18], [19].

\subsection{Anterior Cruciate Ligament}

The ACL is one of the four major stabilizing ligaments of the knee. It attaches to the medial side of the lateral condyle of the femur, traveling distally and anteriorly to attach to the anterior intercondylar eminence of the tibia [20]. The primary function of the $\mathrm{ACL}$ is to prevent excessive anterior tibial translation (ATT), with secondary functions being maintaining varus-valgus stability and prevention of excessive axial rotation [21]. Recent research has shown that there are actually two bundles associated with the ACL, the anteromedial (AM) and posterolateral $(\mathrm{PL})$ [22]. Their names are associate with where each bundle attaches to the tibia. Each bundle has a distinct range of knee flexion where they are most effective: the effective range of the PL bundle is from full extension to a 
flexion angle of about $30^{\circ}$; the effective range of the $\mathrm{AM}$ bundle is a flexion angle greater than $30^{\circ}$ [23]. Tearing of either bundle changes knee kinematics, which changes the loading and stress in the tissue of the articular cartilage which can cause OA [11], [20], [23].

ACL injuries can mean a variety of different things, from a bundle being slightly over stretched to a complete tear of both bundles; in some cases neither bundle tears, however the bone at the attachment site detaches from the rest of the bone. There are two main options for an ACL injured person: 1) physical therapy, and 2) reconstructive surgery followed by physical therapy [24]. Within the surgical solution, there are a variety of different procedures the surgeon can choose to perform such as isometric single bundle (SB) reconstructive surgery (RS), anatomic SB RS and anatomic double bundle (DB) RS [25-28]. Even once the surgery type is chosen, there are still a variety of graft types to choose from: Patellar tendon graft, Hamstring graft, Achilles tendon graft, ACL allograft [2831]. Even with a successful ACL RS it is important to perform proper physical therapy to reduce the likelihood of developing OA.

\subsection{Finite Element Analysis}

The knee is a very complex structure and predicting how the loads propagate during certain activities and due to injuries can be quite difficult. Studies have determined knee loads during various activities but are limited to measuring the articular cartilage stress [32, 33]. A powerful method for predicting how the loads and stresses propagate through the knee joint is to use a finite element model (FEM) of the knee. An FEM is a computational model used when 
physical experiments may be too time consuming, expensive or impossible. In the model, the geometry of the structure is created by assembling "meshed" versions of the underlying components, where each component is modeled as numerous finite elements. Each component then has the appropriate material properties, loading conditions and boundary conditions (BCs) applied to it. Finite element analysis (FEA) uses these conditions and material properties to determine a number of output variables including stress, strain, contact pressure and contact area. FEMs have already been used many times to analyze different components of the knee and overall knee kinematics [34-53] and only some of those have estimated articular cartilage stresses and strains [34-38, 40-42, 48, 51-53]. A previous study modeled ACL grafts as springs to measure the ATT and internal tibial rotation due to variations in graft stiffness using an anterior tibial and quadriceps load [47], while another accurately modeled the ACL DB graft geometry to measure the change in length and tension of a DB reconstruction at various knee flexion angles [46]. However, none have modeled the knee joint using the ACL double-bundle model with realistic geometry or accurately modeled ACL injury and RS to predict articular cartilage stress and strain. It is clear, that to better understand how cartilage loading changes via ACL injuries, $\mathrm{ACL}$ RS and their association with $\mathrm{OA}$, a more complete model must be developed.

\subsection{Objective}

The long term goal of the human motion biomechanics (HMB) group at California Polytechnic State University, San Luis Obispo is to develop a validated 
subject-specific FEM of the human knee to be used in clinical applications to determine patient specific short-term rehabilitative and long-term fitness sustainment exercises that may prevent the onset, or progression, of $O A$ in patients with $A C L$ injured or $A C L$ reconstructed knees. As an initial step to the long term goals of this lab the specific aims of this project are to 1) create and validate a complete FEM of the tibiofemoral joint using a two-bundle model of the ACL and 2) predict articular cartilage tissue loading (contact pressure) for the knee during gait with healthy, $A C L$ injured and $A C L$ reconstructed knees. ACL injuries will be analyzed via parameter studies that 1) reduce the tissue modulus of the $\mathrm{AM}$ and $\mathrm{PL}$ bundles individually or simultaneously or 2) remove the $\mathrm{AM}$ and PL bundles individually or simultaneously. Anatomic SB ACL RS will be analyzed via bone-patellar tendon-bone (BPTB) graft one year post-operation. 


\section{CHAPTER 2 DEVELOPMENT}

\subsection{Knee Structure Development}

The knee structures were modeled from magnetic resonance images (MRIs), then meshed and assembled to form a FEM. Throughout the development stages various aspects of the knee joint were reviewed and approved by an orthopedic surgeon and radiologist.

\subsubsection{Subject Information}

The structures of a right knee were built from 65 sagittal plane MRIs (GE Medical Systems, Ideal GRE, TR $=7.428 \mathrm{~ms}, \mathrm{TE}=4.16 \mathrm{~ms}$, slice spacing $=1.5$ $\mathrm{mm}$, flip angle $=45^{\circ}$, pixel spacing $=.3156$ ) of a healthy, 33 year old male with no prior history of injuries (Table 1). The tissue structures included in the FEM were: femur and tibia bone; medial and lateral menisici; femoral and tibial articular cartilage; $\mathrm{ACL}$, posterior cruciate ligament $(\mathrm{PCL})$, medial collateral ligament (MCL) and lateral collateral ligament (LCL).

Table 1: Subject Information.

\begin{tabular}{|c|c|}
\hline Anatomic Location & Right Knee \\
\hline Subject Age & 33 \\
\hline Subject Height & $5^{\prime} 10^{\prime \prime}$ \\
\hline Subject Weight & $185 \mathrm{lbs}$ \\
\hline Subject Gender & Male \\
\hline
\end{tabular}




\subsubsection{Obtaining Structures}

In each MRI all the tissue and bone structures present were manually shaded, a process called segmenting, to form 2-D masks of that structure using Mimics (Materialise NV, Leuven, Belgium). A rough 2-D mask for each structure was first created using the Mimics "threshold" command that allows the user to select pixels that fall within a user-defined grey-scale intensity. The masks were then refined manually using the erase and draw tools. The 2-D masks were reviewed by an orthopedic surgeon and a radiologist to ensure proper selection of the particular structure.

Once all the images were properly segmented, the 2-D masks for a given structure were combined to form a 3-D object. For every structure except the tibia and femur, the 3-D object was created using the "high quality" generation. Due to the fact that the MRIs are only an incremental view of the knee, some 3-D objects had sharp edges that would prove to be difficult in the meshing process; these edges were softened with Mimics' built in Gaussian smoothing function with a smoothing factor of .8 and 500 iterations for the tibia and femur and a smoothing factor of .8 and 200 iterations for all other structures. To ensure that the smoothing process didn't change the 3-D object too much, 2-D masks of the smoothed 3-D objects were made and compared to the original 2-D masks of each structure. When the 3-D object was shown to properly represent the given structure, the surface of the 3-D object was saved as a .stl file and imported into SolidWorks (Dassault Systemes, Velizy-Villacoublay, France). Figure 1 shows an 
example of the MRIs with and without the ACL mask and the resulting smoothed 3-D object of the ACL.
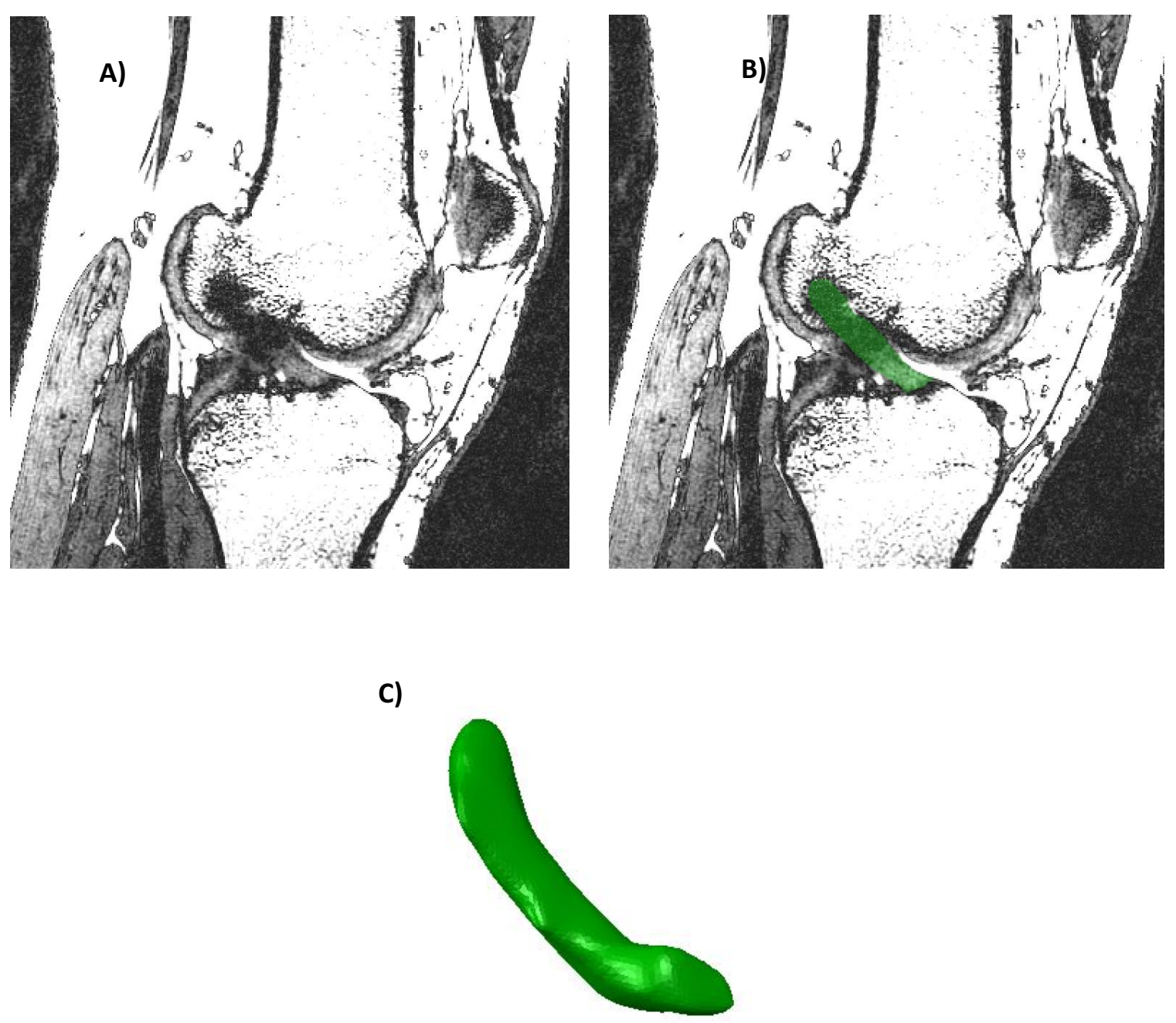

Figure 1: MR image with A) No mask. B) ACL mask. C) Resulting 3-D object of the ACL.

Each .stl file was opened in SolidWorks as a mesh file using the "ScanTo3D" add-in. In the case of the articular cartilage and menisci, the structures had tightly curved edges which could cause problems meshing the 
structures accurately. To avoid these problems before they arose, these curved edges were flattened. This was done by selecting the curved edge portion of the mesh and deleting it. Doing so left two separate meshes that represented the top and bottom surfaces of the structures. The edges of the two surfaces were then smoothed to enable a surface connecting the top and bottom surfaces to be created using the surface loft tool. This process is shown in Figure 2.
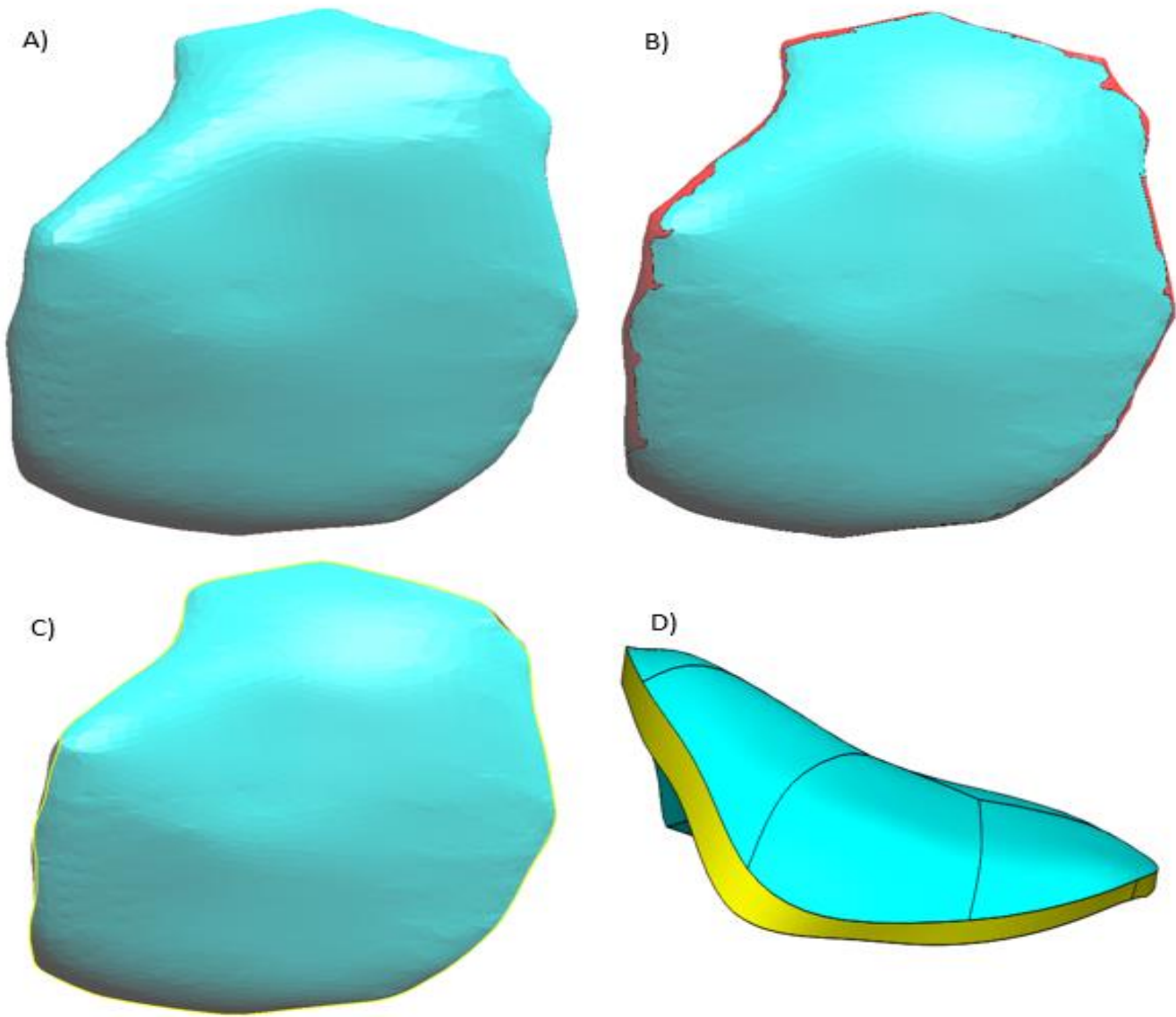

Figure 2: Lateral Tibial Cartilage: A) Surface Mesh Imported from Mimics. B) Selected Curved Edges to be deleted. C) Smoothed Surface Edges after Curved Edges have been deleted. D) Flat Surface Lofted connecting the Smoothed Surface Edges. 
The PCL, tibia and femur did not have tightly curved edges that needed to be flattened. However, if there were any sharp points that the smoothing process in Mimics missed they were smoothed while the structure was still a mesh, using the "Mesh Prep Wizard" in SolidWorks' "ScanTo3D" add-in. The sharp points were deleted and the resulting hole was replaced with a smooth surface automatically generated using the "Mesh Prep Wizard". This process is shown in Figure 3. Once all the sharp points were smoothed and curved edges flattened, the meshed structures were converted into solids for partitioning and assembly.

A)

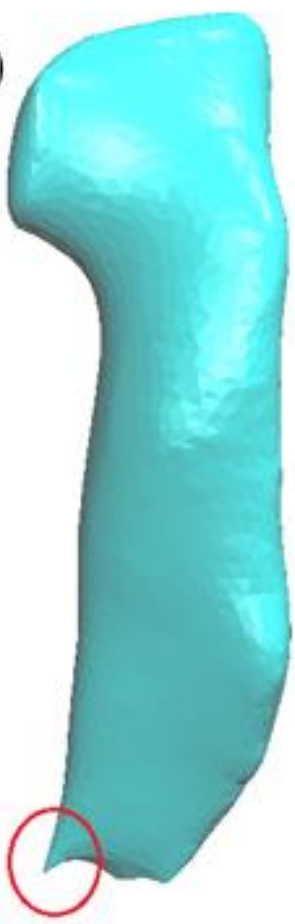

B)

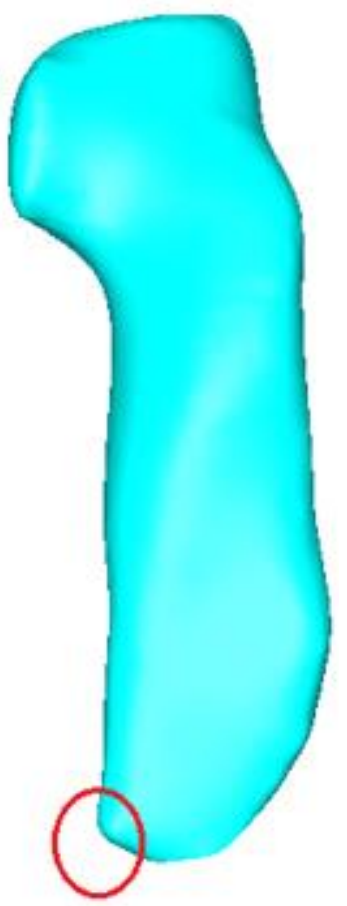

Figure 3: PCL Before $(A)$ and After $(B)$ sharp points were smoothed.

The PCL, tibia, femur, articular cartilage and menisci were then assembled together to form the knee joint. Slight overlaps between the bones, articular cartilage and menisci appeared once the knee was assembled that 
would cause the finite elements to excessively distort during the analysis which could prevent the FE solver from converging to a solution. It was decided that any overlap with bone and articular cartilage would be fixed by removing the overlapped volume from the articular cartilage. For menisci and articular cartilage overlap, the overlapped volume would be removed from the menisci. During this process, sharp corners were occasionally created after the overlaps were removed. These sharp corners were removed in SolidWorks by creating surfaces to cut them away. An example of this can be seen in Figure 4 .

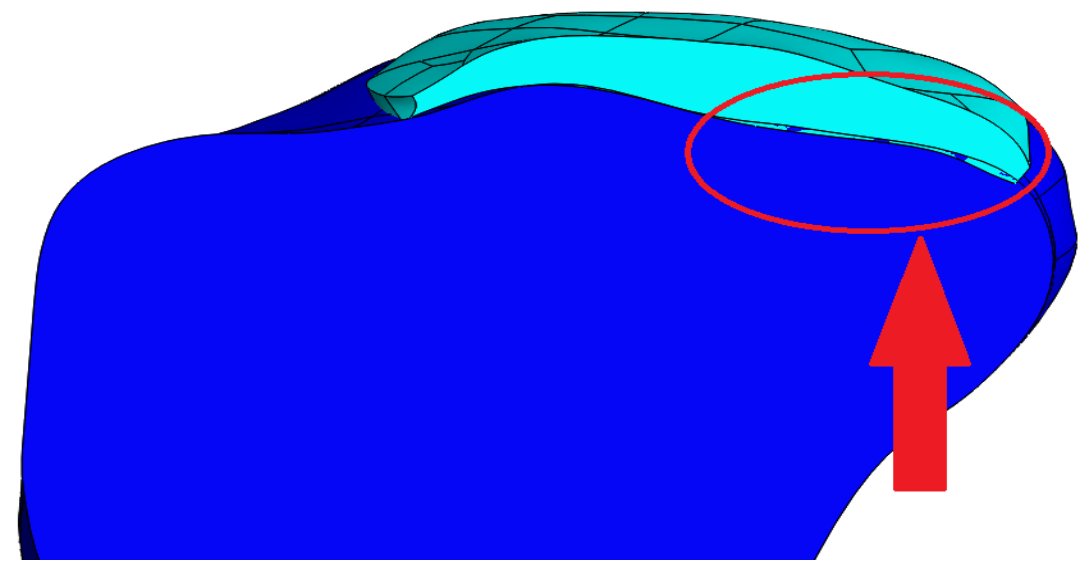

Figure 4: Example of slight overlap between tibia and lateral tibial cartilage.

The AM and PL bundles were not imported into SolidWorks from Mimics because it is extremely difficult to decipher the separation of the two bundles on MRIs. This meant that the AM and PL bundles had to be manually built in SolidWorks from published data on their attachment sizes and locations [54-57]. The AM and PL bundle femoral attachment sites were modeled as circles with a radius of 3.6 and $3.3 \mathrm{~mm}$, respectively. The AM and PL bundle tibial attachment sites were modeled as ellipses with major axis half-lengths of 6.45 and $5.89 \mathrm{~mm}$, 
respectively, and minor axis half-lengths of 3.3 and $2.8 \mathrm{~mm}$, respectively. A more detailed anatomical description of the AM and PL bundles can be seen in Table 2. The two bundles were only considered acceptable after the orthopedic surgeon approved their location, attachment size, and volume.

Table 2: Anatomical description of AM and PL bundles [54-57].

\begin{tabular}{|c|c|c|}
\hline & AM Bundle & PL Bundle \\
\hline Femoral attachment radius & $3.6 \mathrm{~mm}$ & $3.3 \mathrm{~mm}$ \\
\hline Center to center distance of Femoral sites of AM to PL & N/A & $7.0 \mathrm{~mm}$ \\
\hline Angle from vertical of Femoral sites from AM to PL centers & N/A & $12.0^{\circ}$ \\
\hline Femoral attachment area & $42.4 \mathrm{~mm}^{2}$ & $36.0 \mathrm{~mm}^{2}$ \\
\hline Tibial attachment major axis half length & $6.45 \mathrm{~mm}$ & $5.89 \mathrm{~mm}$ \\
\hline Tibial attachment minor axis half length & $3.3 \mathrm{~mm}$ & $2.8 \mathrm{~mm}$ \\
\hline Tibial attachment area & $71.3 \mathrm{~mm}{ }^{2}$ & $55.7 \mathrm{~mm}^{2}$ \\
\hline Lateral center-to-center dist. of AM to PL tibial attachment & $\mathrm{N} / \mathrm{A}$ & $1.8 \mathrm{~mm}$ \\
\hline Posterior center-to-center dist. of AM to PL tibial attachment & $\mathrm{N} / \mathrm{A}$ & $6.3 \mathrm{~mm}$ \\
\hline Volume & $1113.1 \mathrm{~mm}^{3}$ & $640.0 \mathrm{~mm}^{3}$ \\
\hline
\end{tabular}

Since the MRIs were taken from a healthy individual, the BPTB graft had to be modeled in SolidWorks. As mentioned earlier, the surgery type modeled in this study is an anatomic SB RS using a BPTB graft. An anatomic SB RS is named as such because the femoral and tibial attachment sites of the graft are located in between the anatomical locations of the AM and PL bundle attachments. It has been reported in goats and rabbits that at one year postoperation the graft is similar in size to the intact ACL [58], [59]. With this 
information it was decided that the size and attachment locations of the BPTB graft would be similar to an intact $\mathrm{ACL}[54,56]$. The femoral attachment was modeled as an ellipse with a major axis half-length of $7.01 \mathrm{~mm}$ and a minor axis half-length of $4.23 \mathrm{~mm}$. The tibial attachment was modeled as a circle with a radius of $6.20 \mathrm{~mm}$. A more detailed anatomical description of the BPTB graft can be seen in Table 3. The BPTB graft was only considered acceptable after the orthopedic surgeon approved the location, attachment size, and volume.

Table 3: Anatomical description of the BPTB graft $[54,56,58,59]$.

\begin{tabular}{|c|c|}
\hline Femoral attachment major axis half length & $7.01 \mathrm{~mm}$ \\
\hline Femoral attachment minor axis half length & $4.23 \mathrm{~mm}$ \\
\hline Angle from vertical of Femoral attachment & $11.0^{\circ}$ \\
\hline Femoral attachment area & $96.04 \mathrm{~mm}^{2}$ \\
\hline Tibial attachment radius & $6.20 \mathrm{~mm}$ \\
\hline Tibial attachment area & $126.84 \mathrm{~mm}^{2}$ \\
\hline Volume & $1731.20 \mathrm{~mm}^{3}$ \\
\hline
\end{tabular}

The complete structure of the MCL and LCL are often times not seen in MRIs, as was the case with this subject. This meant that both ligaments had to be manually built in SolidWorks from published data on their attachment sizes and locations [60-65]. Because the fibula is not included in this FEM, the fibular attachment of the LCL was not modeled. Instead, the LCL was cut in half with the distal face of the LCL sized to the average size of an LCL. The femoral attachment of the LCL and MCL were modeled as ellipses with major axis half- 
lengths of 4.81 and $6.80 \mathrm{~mm}$, respectively, and minor axis half-lengths of 3.35 and $3.75 \mathrm{~mm}$, respectively. The tibial attachment of the MCL was also modeled as an ellipse with a major axis half-length of $16.34 \mathrm{~mm}$ and a minor axis halflength of $10.10 \mathrm{~mm}$. A more detailed anatomical description of the LCL and MCL can be seen in Table 4 and Table 5, respectively. The two ligaments were only considered acceptable after the orthopedic surgeon approved their location, attachment size, and volume.

Table 4: Anatomical description of the LCL [60-65].

\begin{tabular}{|c|c|}
\hline Femoral attachment major axis half length & $4.81 \mathrm{~mm}$ \\
\hline Femoral attachment minor axis half length & $3.35 \mathrm{~mm}$ \\
\hline Femoral attachment area & $50.6 \mathrm{~mm}^{2}$ \\
\hline Distal section major axis half length & $2.13 \mathrm{~mm}$ \\
\hline Distal section minor axis half length & $1.08 \mathrm{~mm}$ \\
\hline Distal section area & $7.23 \mathrm{~mm}^{2}$ \\
\hline Length from top of femoral attachment to distal section & $31.51 \mathrm{~mm}$ \\
\hline Volume & $227.67 \mathrm{~mm}^{3}$ \\
\hline
\end{tabular}

Table 5: Anatomical description of the MCL [60-65].

\begin{tabular}{|c|c|}
\hline Femoral attachment major axis half length & $6.80 \mathrm{~mm}$ \\
\hline Femoral attachment minor axis half length & $3.75 \mathrm{~mm}$ \\
\hline Femoral attachment area & $80.24 \mathrm{~mm}^{2}$ \\
\hline Tibial attachment major axis half length & $16.34 \mathrm{~mm}$ \\
\hline
\end{tabular}




\begin{tabular}{|c|c|}
\hline Tibial attachment minor axis half length & $10.10 \mathrm{~mm}$ \\
\hline Tibial attachment area & $270.68 \mathrm{~mm}^{2}$ \\
\hline Center-to-center length & $80.73 \mathrm{~mm}$ \\
\hline Volume & $4592.91 \mathrm{~mm}^{3}$ \\
\hline
\end{tabular}

After all the ligaments were built and overlaps removed, the entire knee joint assembly was once again reviewed by the orthopedic surgeon before any further work was done. The approved SolidWorks knee joint can be seen in Figure 5. 

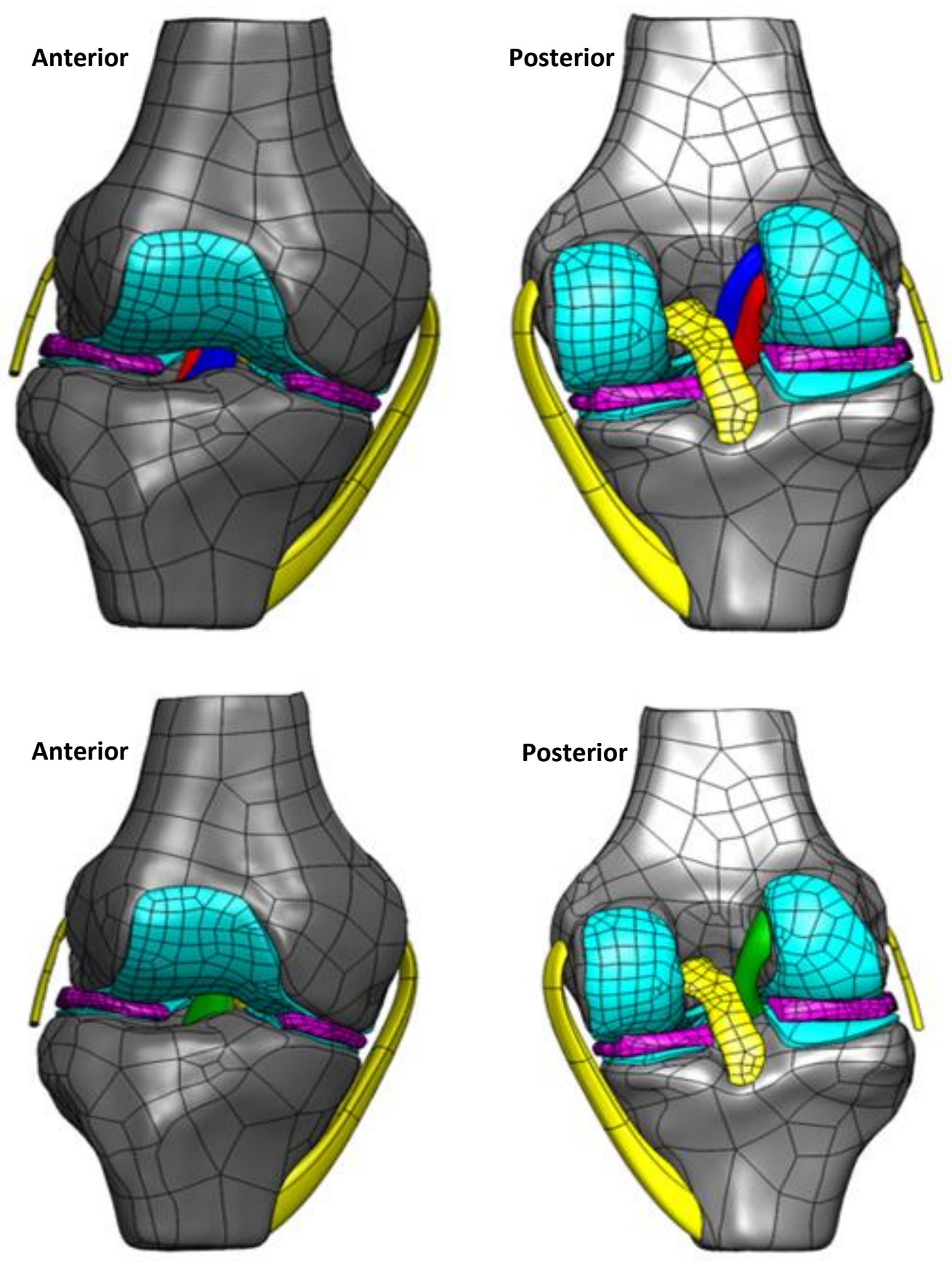

Figure 5: Anterior and Posterior view of assembled knee joint. Ligaments are yellow, bone is grey, articular cartilage is cyan, menisci are purple, AM bundle is blue, PL bundle is red, BPTB graft is green. 


\subsubsection{Partitioning Structures}

After some trial and error it was discovered that partitioning the structures was easily done in SolidWorks. Partitions are curves used to apply the best possible mesh to a part. The purpose of the part affected the way the part was partitioned; bones, which were modeled as rigid bodies (see Section 2.2.1), only had partition curves applied to the surface of the part; soft tissue structures, not modeled as rigid bodies (see Section 2.2.1), had partition curves applied to both the surface and the inside of the part, Figure 6.

A)

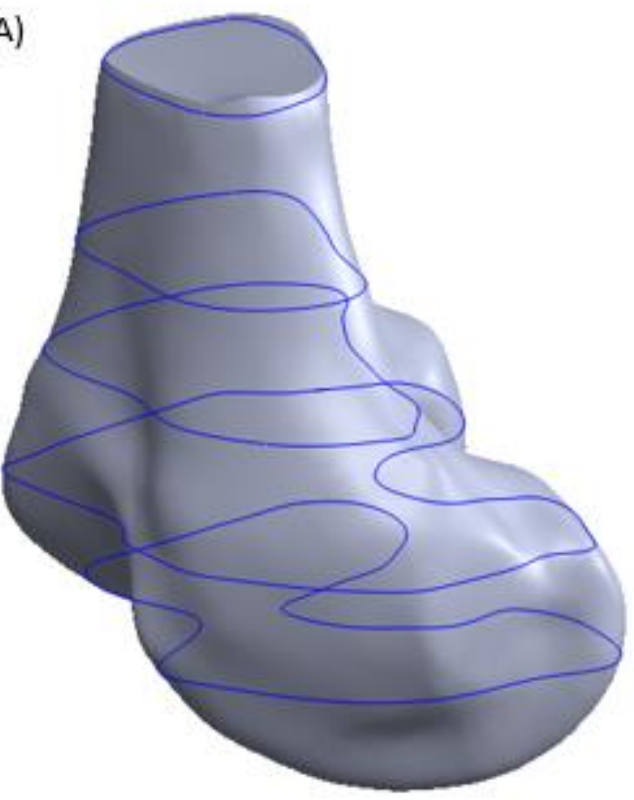

B)

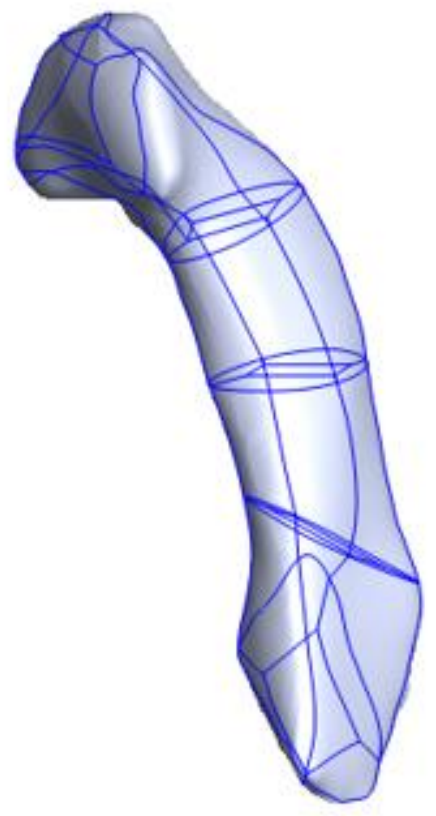

Figure 6: A) Femur with partitioning lines used for meshing. B) PCL with partitioning lines used for meshing.

The partitioning curves of the femur and tibia were created by projecting 2D curves onto their respective surfaces. For the soft tissue structures, planes 
were created to cut up the structure into smaller solid structures. The partitioning curves were then applied to the faces of the cut portions. Any partition curves not on the cut faces were created by projecting 2-D curves onto the surface of the structure. The locations of the partitioning curves were decided after meshing trial and error. After a structure was partitioned, it was saved as a .igs file and exported to TrueGrid (XYZ Scientific Applications, Inc., Livermore, California, USA).

\subsubsection{Meshing Structures}

TrueGrid is a meshing program that allows the user to create meshes of geometries that have been imported into or built in TrueGrid, then export those meshes to various FE programs. The "iges" command opens igs files and automatically creates and numbers surfaces, edges and curves from the imported geometry. The faces and edges of computational blocks created using the "block" command, can then be projected onto the surfaces, edges and curves created from the "iges" command.

As mentioned earlier the bones only had partitioning curves applied to the surface, this is because they were meshed using 3-D surface elements, R3D4. To do this, a 2-D array of elements were created using the block command then trimmed using the "dei" command. They were trimmed in a way that would best wrap around the bone. The computational edges and faces were then projected to the appropriate curves and surfaces using the "cure" and "sfi" commands, respectively. The computational blocks were then refined to better map the 
curves and surfaces using the "mseq" command. An example of this involving the tibia can be seen in Figure 7.
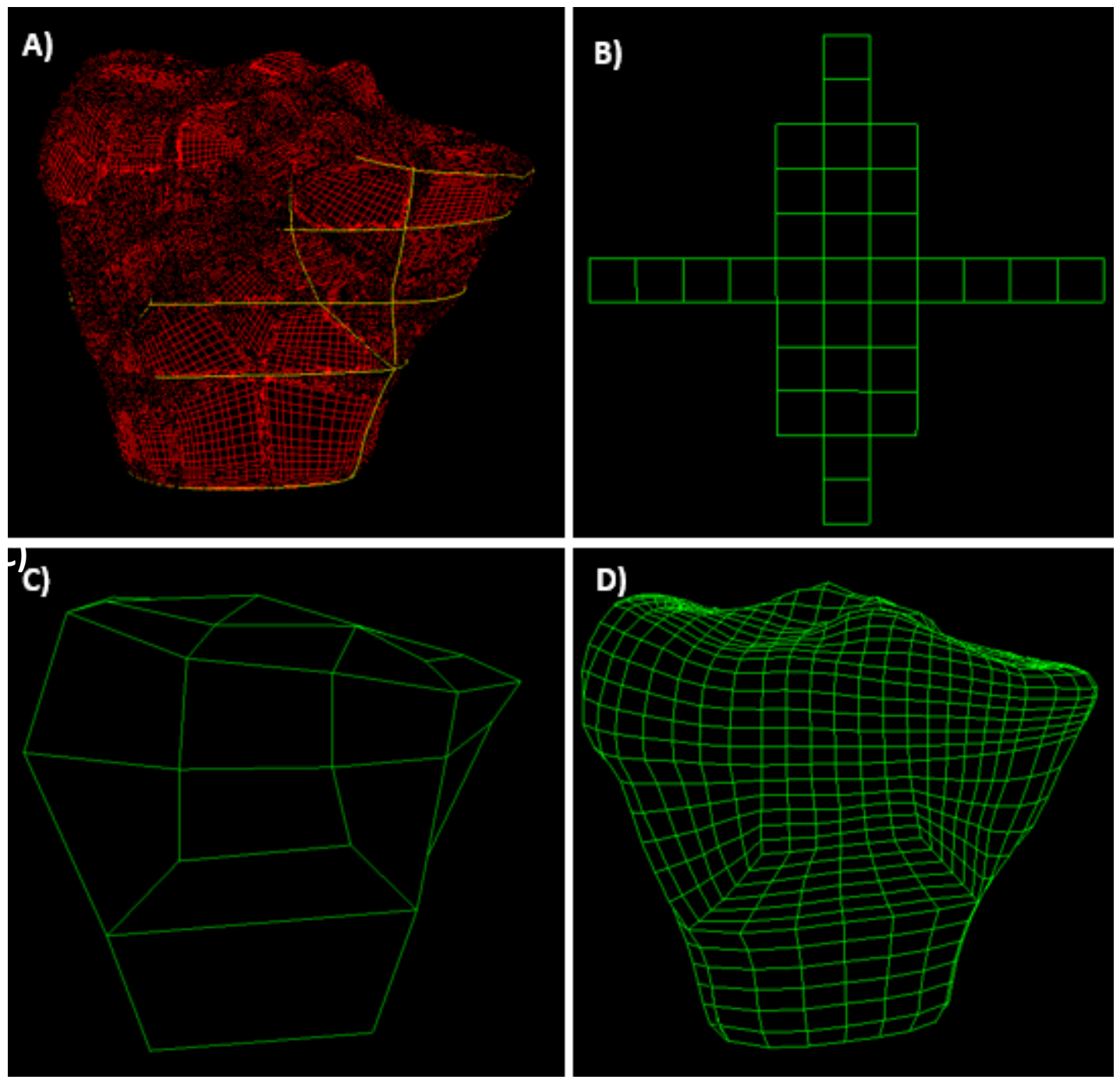

Figure 7: A) Surfaces and curves of the tibia created from "iges" command. B) Trimmed 2-D array of computational blocks created with "block" command. C) Unrefined projection of computational blocks onto tibia geometry. D) Refined projection of computational blocks onto tibia geometry. 
A similar process was done with the soft tissue structures, except that the complexity level was increased because they required a 3-D array of computational blocks to properly mesh each structure. The soft tissue structures were meshed using linear brick elements, C3D8. An example of this involving the PL bundle can be seen in Figure 8. 

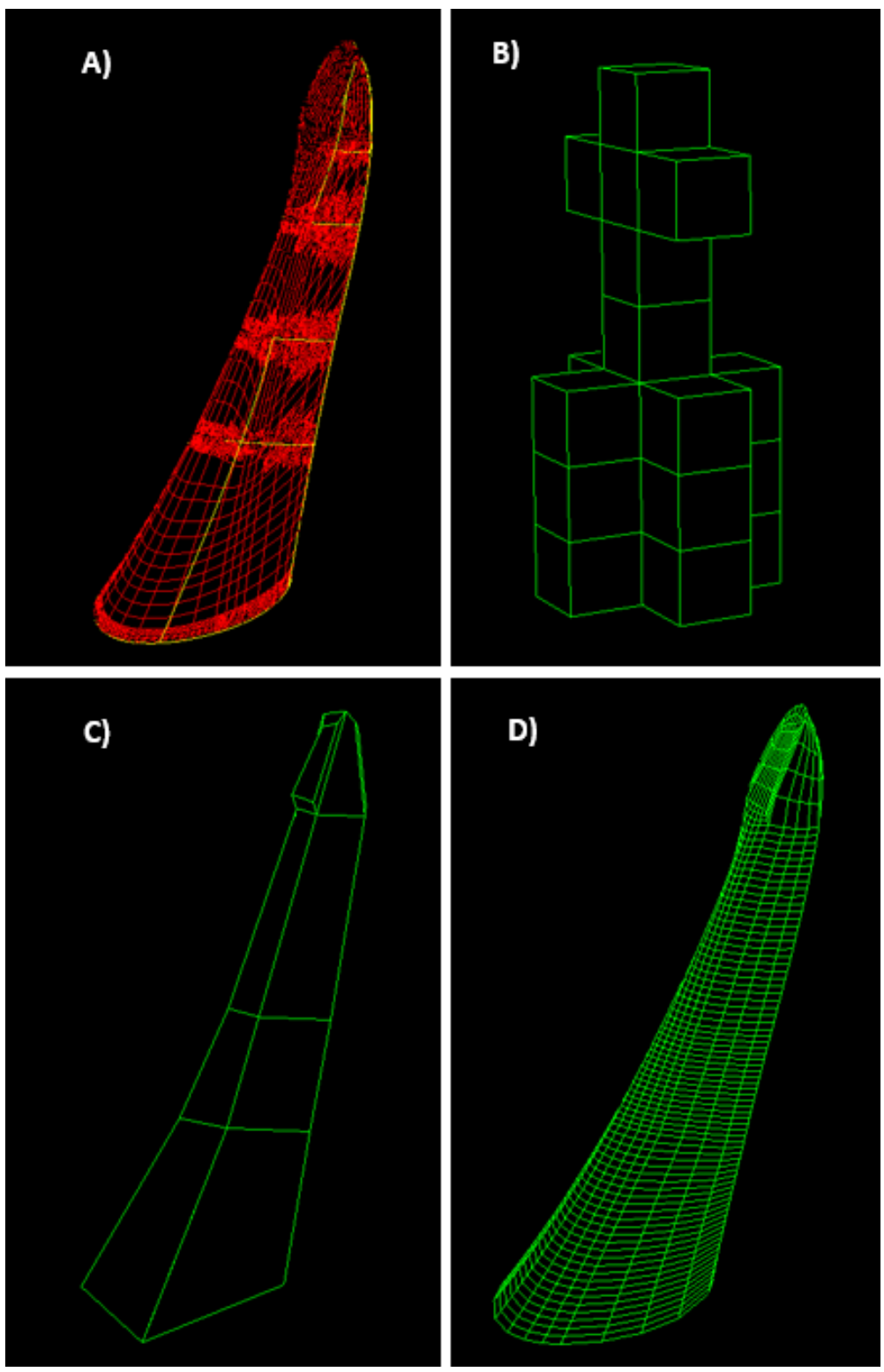

Figure 8: A) Surfaces and curves of the PL bundle created from "iges" command.

B) Trimmed 3-D array of computational blocks created with "block" command. C) Unrefined projection of computational blocks onto PL geometry. D) Refined projection of computational blocks onto PL geometry.

The "merge" command was then used to remove any free edges in the meshed part. Free edges occur when faces or edges of two different 
computational blocks are projected to the same surface or curve, but nothing is defined to tell the FE solver that these faces or edges are actually representing the same thing. Once finished, the meshed structure was saved as a inp file and exported to Abaqus (Dessault Systemes, Velizy-Villacoublay, France) for assembly and analysis of the FEM. The knee joint assembled with meshed structures in Abaqus can be seen in Figure 9. 

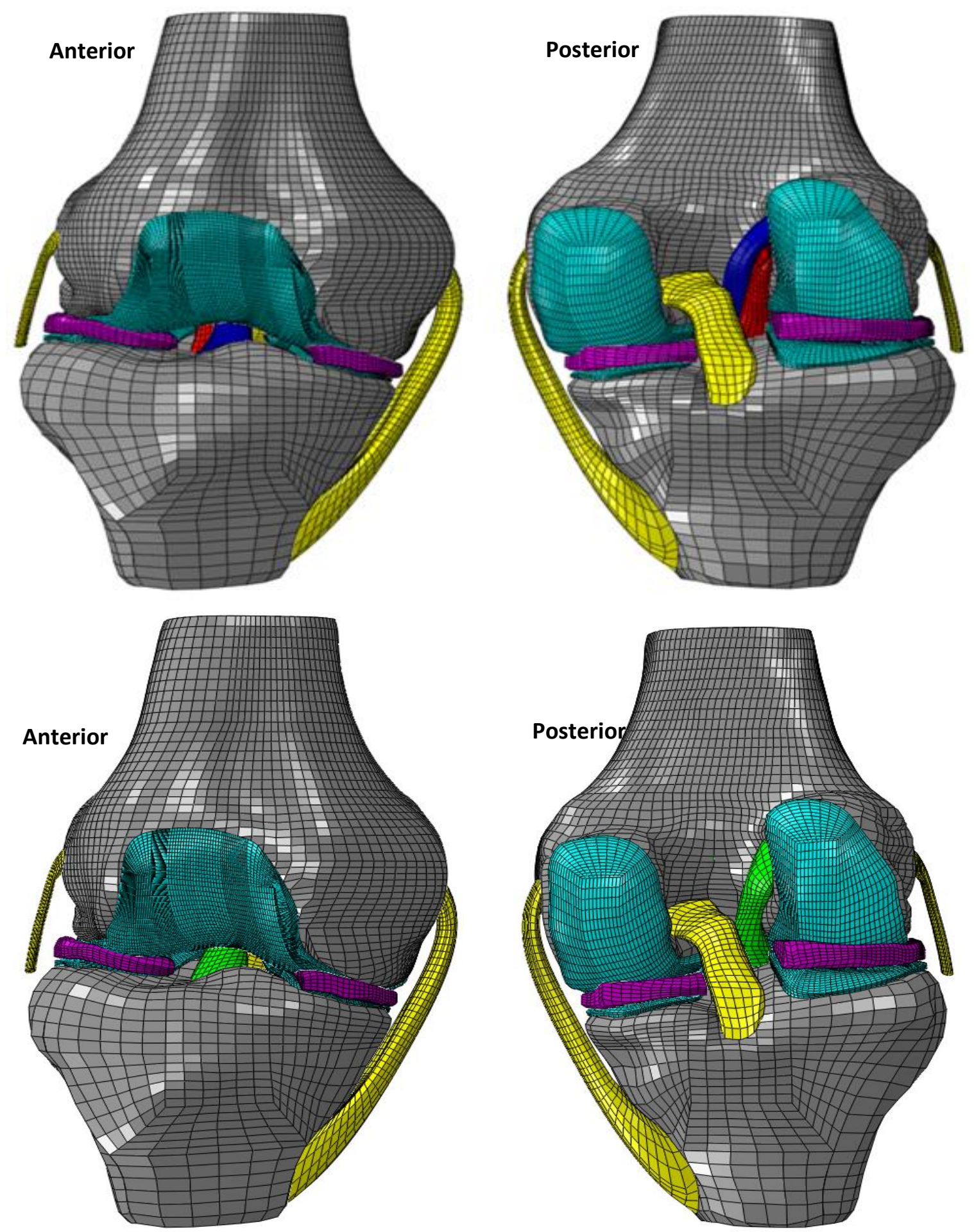

Figure 9: Anterior and Posterior view of meshed knee joint. Ligaments are yellow, bone is grey, articular cartilage is cyan, menisci are purple, AM bundle is blue, PL bundle is red, BPTB graft is green. 


\subsection{Finite Element Model Development}

Abaqus is a FE solver that offers both pre- and post-processing capabilities. The pre-processing capabilities used include material definition, contact interaction, and boundary and loading conditions. The post-processing capabilities were used to analyze the results of the validation and gait study. The standard/implicit Abaqus solver with non-linear geometry was used for each analysis.

\subsubsection{Material Definition}

As mentioned earlier the tibia and femur were modeled as rigid bodies. This method has been done in previous FE studies because of the high stiffness of bone when compared to other soft tissue $[34,42,49]$. It has been shown to only have a marginal effect on the predicted stresses and strains within soft tissue structures when compared to models using more complicated bone material definitions [43]. The bones were defined as solids by defining the imported mesh as a 3-D, discrete rigid body in the Part module. Doing so meant that the bones' motions are only described with rigid body translations and rotations.

The articular cartilage was modeled as a linearly elastic, homogeneous, isotropic material with a Young's modulus (E) of $15 \mathrm{MPa}$ and a Poisson's ratio (v) of .475 , equivalent to previous studies $[43,66]$. In reality articular cartilage is more accurately modeled as a biphasic, poroviscoelastic material [67]. The mechanical structure consists mainly of a collagen fibril network, whose orientation and concentration are both depth-dependent, and proteoglycans [68], 
[69]. However, modeling the articular cartilage as a linearly elastic, homogenous, isotropic material is adequate for modeling the almost instantaneous load from gait because the viscoelastic time constant approaches 1500s [70].

The menisci were also modeled as linearly elastic, homogeneous, isotropic materials with a Young's modulus $(\mathrm{E})$ of $50 \mathrm{MPa}$ and a Poisson's ratio (v) of .49 , equivalent to previous studies [41, 42]. As with the articular cartilage, the menisci are much more complicated than this, showing distinct transversely isotropic, visco- and poroelastic material characteristics [71]. One of the viscoelastic characteristics is a large time constant, which allows a linearly elastic, homogeneous, isotropic model to be adequate for the short loading times seen in gait.

The ligaments were modeled as linearly elastic, transversely isotropic materials with the longitudinal direction aligned with the ligament length. The ACL, PCL, LCL and MCL have been shown to have very similar properties, even being reported as having the same material properties $[72,73]$. It has also been shown that the AM and PL bundles have very different properties [74]. Using this information, it was decided that the four ligaments would be modeled using the same material properties. However, the longitudinal and transverse tensile moduli for the ACL were considered effective moduli used to calculate the moduli of the AM and PL bundle based off of the volume ratios of the bundles. 


$$
\begin{aligned}
& E_{L}^{A M}=\frac{V^{A M}}{V^{A C L}} E_{L}^{A C L} \\
& E_{T}^{A M}=\frac{V^{A M}}{V^{A C L}} E_{T}^{A C L} \\
& G_{L T}^{A M}=\frac{V^{A M}}{V^{A C L}} G_{L T}^{A C L} \\
& E_{L}^{P L}=\frac{V^{P L}}{V^{A C L}} E_{L}^{A C L} \\
& E_{T}^{P L}=\frac{V^{P L}}{V^{A C L}} E_{T}^{A C L} \\
& G_{L T}^{P L}=\frac{V^{P L}}{V^{A C L}} G_{L T}^{A C L}
\end{aligned}
$$

Reported values of the transverse-transverse shear modulus could not be found, so the one used was calculated by equating the ratio of it with the longitudinal-transverse shear modulus to the ratio of the transverse tensile modulus with the longitudinal tensile modulus.

$$
\begin{gathered}
G_{T T}^{A M}=\frac{E_{T}^{A C L}}{E_{L}^{A C L}} G_{L T}^{A M} \\
G_{T T}^{P L}=\frac{E_{T}^{A C L}}{E_{L}^{A C L}} G_{L T}^{P L}
\end{gathered}
$$

The longitudinal-transverse Poisson's ratio was taken from reported values of hip ligament Poisson's ratio. The final material properties were averaged from available published data, based on the total number of specimen in all the studies [72-78]. When modeling an AM bundle injury, PL bundle injury 
or total ACL injury, the longitudinal and transverse moduli of the affected bundle were reduced by $50 \%$. A complete list of the material properties used for the ligaments are shown in Table 6.

The patellar tendon can be modeled accurately as a linearly elastic, transversely isotropic material with a longitudinal tensile modulus that is much larger than the $A C L, L C L, M C L$ and PCL $[72,79,80]$. Despite this fact, when BPTB grafts in cadaver knees are analyzed they consistently have a tensile stiffness and modulus much lower than the intact ACL [81-83]. This trend isn't unique to BPTB grafts, similar results have been reported for Achilles tendon grafts [84-85]. The mechanical properties of the graft are typically lowest soon after the surgery, gradually increasing until they level off to roughly $40 \%$ of the intact ACL at one year following the operation. Since the BPTB graft modeled is one year post-operation, its material characteristics were determined by reducing the ligament material characteristics by $60 \%$. A complete list of the material properties used for the BPTB graft are shown in Table 6. 
Table 6: Ligament Properties [72, 79-85].

\begin{tabular}{|c|c|c|c|c|c|c|}
\hline & $\begin{array}{l}\mathrm{LCL} / \mathrm{MCL} / \\
\mathrm{PCL}\end{array}$ & $\begin{array}{l}\text { AM } \\
\text { bundle }\end{array}$ & $\begin{array}{c}\mathrm{PL} \\
\text { bundle }\end{array}$ & BPTB & $\begin{array}{l}\text { AM } \\
\text { Injury }\end{array}$ & $\begin{array}{c}\mathrm{PL} \\
\text { Injury }\end{array}$ \\
\hline Longitudinal Modulus $\left(\mathrm{E}_{\mathrm{L}}\right)(\mathrm{MPa})$ & 153.7 & 212.2 & 115.6 & 61.5 & 106.1 & 57.8 \\
\hline Transverse Modulus $\left(\mathrm{E}_{\mathrm{T}}\right)(\mathrm{MPa})$ & 5.1 & 7.1 & 3.9 & 2.04 & 3.5 & 1.9 \\
\hline $\begin{array}{l}\text { Longitudinal-Transverse Shear } \\
\text { Modulus }\left(\mathrm{G}_{\mathrm{LT}}\right)(\mathrm{MPa})\end{array}$ & 1.7 & 1.7 & 1.7 & .68 & 1.7 & 1.7 \\
\hline $\begin{array}{c}\text { Transverse-Transverse Shear } \\
\text { Modulus (GTT) (MPa) }\end{array}$ & .06 & .06 & .06 & .02 & .06 & .06 \\
\hline $\begin{array}{l}\text { Longitudinal-Transverse Poisson's } \\
\text { ratio }\left(\mathrm{v}_{\mathrm{LT}}\right)\end{array}$ & 1.4 & 1.4 & 1.4 & 1.4 & 1.4 & 1.4 \\
\hline $\begin{array}{c}\text { Transverse-Transverse Poisson's } \\
\text { ratio }\left(\mathrm{v}_{\mathrm{TT}}\right)\end{array}$ & .3 & .3 & .3 & .3 & .3 & .3 \\
\hline
\end{tabular}

\subsubsection{Contact Definition}

There were 10 contact pairs defined between structures using a surfaceto-surface contact:

1. Femoral cartilage and lateral meniscus

2. Femoral cartilage and lateral tibial cartilage

3. Lateral tibial cartilage and lateral meniscus

4. Femoral cartilage and medial meniscus

5. Femoral cartilage and medial tibial cartilage

6. Medial tibial cartilage and medial meniscus

7. AM bundle and PL bundle (when applicable) 
8. AM bundle and PCL (when applicable)

9. PL bundle and PCL (when applicable)

10. BPTB graft and PCL (when applicable)

For the cases of contact between articular cartilage and articular cartilage or articular cartilage and meniscus a hard-contact, frictionless, finite sliding formulation was used, similar to previous FE studies [42], [43], [86]. For the remaining cases a hard-contact, frictionless, small sliding formulation was used.

\subsubsection{Constraint Definition}

The 12 tie constraints defined to represent the attachment of soft tissue to bone and their discretization method were:

1. Femur and AM bundle (Node-to-surface)

2. Femur and femoral cartilage (Surface-to-surface)

3. Femur and LCL (Analysis default)

4. Femur and MCL (Analysis default)

5. Femur and PCL (Analysis default)

6. Femur and PL bundle (Node-to-surface)

7. Tibia and AM bundle (Node-to-surface)

8. Tibia and lateral tibial cartilage (Analysis default)

9. Tibia and MCL (Node-to-surface)

10. Tibia and medial tibial cartilage (Analysis default)

11. Tibia and PCL (Analysis default)

12. Tibia and PL bundle (Analysis default) 
In all constraints the bone was defined as the master surface. For Abaqus/Standard the analysis default is surface-to-surface unless the following circumstances are met:

1. If either of the surfaces being tied is node-based

2. If the projection along the slave surface normal direction does not intersect the master surface

3. If single-sided slave and master surfaces have surface normal in approximately the same direction

The surface-to-surface formulation minimizes numerical noise for tied interfaces involving mismatched meshes because it enforces the constraint over a finite region rather than at discrete points. This method was used when none of the nodes on the slave surface started too far from the master surface. The node-to-surface discretization was used when some of the slave nodes were initially too far from the master surface. The analysis default discretization was used when it was uncertain if the surface-to-surface or node-to-surface discretization would produce less difficulties for Abaqus.

\subsubsection{Meniscal Attachment Constraints}

Each meniscal attachment horn was modeled with a single linear spring element connecting the central node on the meniscal attachment face to a node on the tibia. The attachments of each spring can be seen in Figure 10. The spring used was type "SPRINGA" which strictly uses the change in length in the spring between the initial and deformed configurations to determine the spring displacement. The stiffness's of the springs used in each attachment horn were 
taking from published data of the attachment horn's stiffness [87], shown in Table 7.

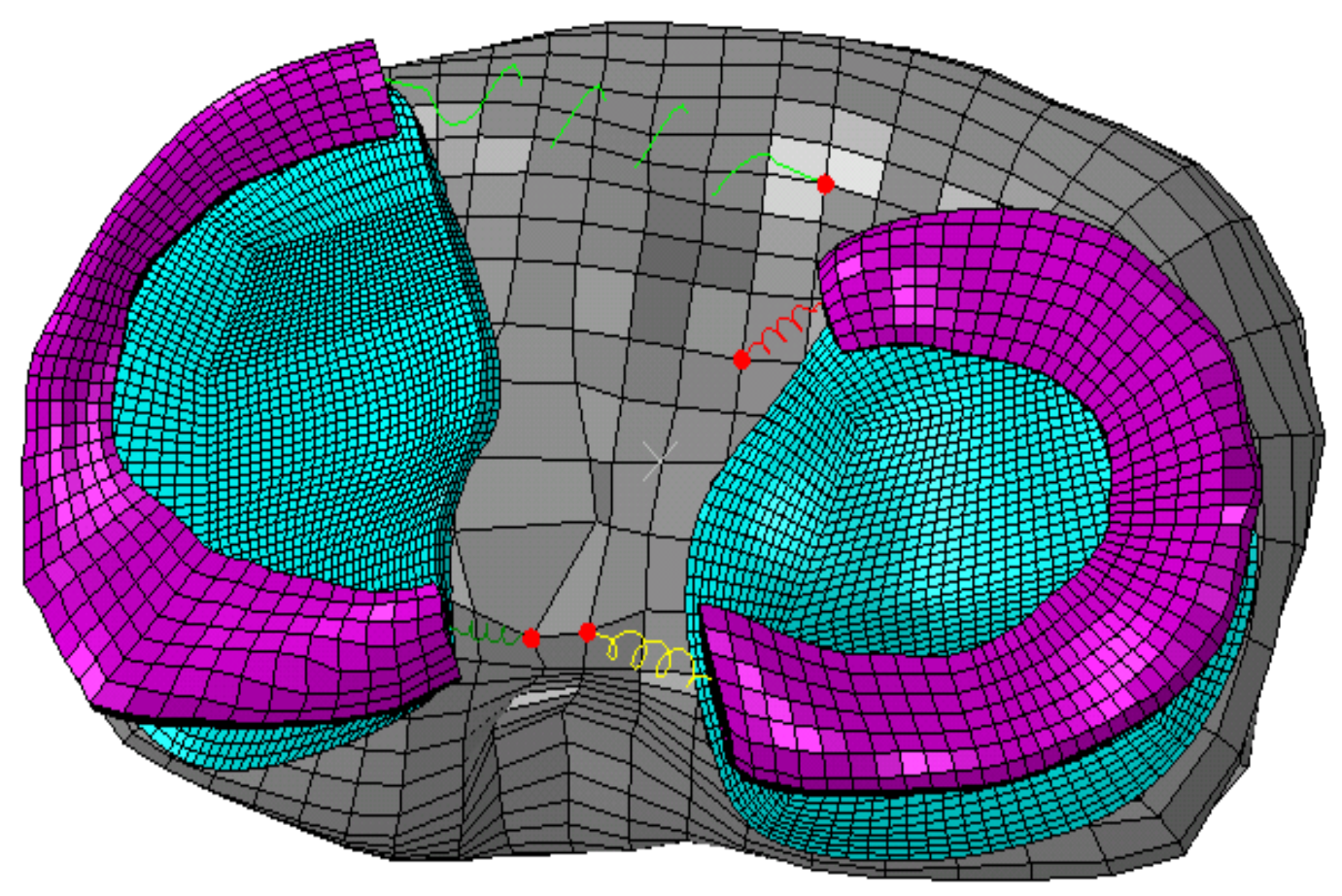

Figure 10: Meniscal Attachment Springs. Medial Anterior Horn is light green, Medial Posterior Horn is dark green, Lateral Anterior Horn is red, and Lateral Posterior Horn is yellow.

Table 7: Meniscal Attachment Horn Stiffness [34].

\begin{tabular}{|c|c|}
\hline Attachment Location & Horn Stiffness (N/mm) \\
\hline Lateral Anterior Meniscus Horn & 215.8 \\
\hline Lateral Posterior Meniscus Horn & 129.5 \\
\hline Medial Anterior Meniscus Horn & 169.4 \\
\hline Medial Posterior Meniscus Horn & 207.2 \\
\hline
\end{tabular}


The rest of the nodes on the meniscal attachment face were tied to the central node using a kinematic coupling constraint. The kinematic coupling constrains the motion of the nodes on the meniscal attachment face to follow the rigid body motion of the central node.

\subsubsection{Lateral Collateral Ligament Distal Face Constraints}

As mentioned earlier, the LCL was cut in half because the fibula was not included in this model. This meant that constraints had to be attached to the distal portion of the $L C L$ to prevent it from inaccurately moving. A rotational $B C$ was applied to all the nodes on the distal face of the LCL, fixing the three rotational degrees of freedom (DOFs). The translational DOFs were constrained by attaching a spring element in each of the three translational directions to each node on the distal surface. The direction of each spring was defined using the Knee Alignment coordinate system (see Section 2.2.6), with the longitudinal spring in the proximal/distal direction and the two transverse springs in the anterior/posterior and medial/lateral directions. The springs used were type "SPRING1" which are only defined using one node. The relative change in length of the spring is computed from displacement of each node in the spring's specified direction. The stiffness of the longitudinal springs applied to each node

were taken from the reported stiffness of knee ligaments $[75,76]$ divided by the total number of nodes on the distal surface (96), while the stiffness of the transverse springs were determined by maintaining the same ratio seen in longitudinal modulus to transverse modulus, Table 8. 
Table 8: LCL Longitudinal and Transverse Spring Stiffness [75, 76].

\begin{tabular}{|c|c|c|}
\hline $\begin{array}{c}\text { LCL Spring } \\
\text { direction }\end{array}$ & $\begin{array}{c}\text { Ligament Stiffness } \\
(\mathrm{N} / \mathrm{mm})\end{array}$ & $\begin{array}{c}\text { Applied Spring Stiffness } \\
(\mathrm{N} / \mathrm{mm})\end{array}$ \\
\hline Longitudinal & 255 & 2.66 \\
\hline Transverse & 8.5 & .089 \\
\hline
\end{tabular}

\subsubsection{Knee Alignment Orientation}

A local Knee Alignment orientation was built to ensure that loads and BCs were applied in their intended directions. In Abaqus a local Cartesian coordinate system is one that is described by three perpendicular axes but can have the origin placed anywhere after the coordinate system is defined. A temporary origin was defined at a node on the tibial plateau, then a datum axis representing the medial/lateral direction was built using the origin and another node that appeared to be purely medial from the origin based off of observations made of the tibial plateau. A datum axis representing the anterior/posterior direction was built using the origin and another node that appeared to be purely anterior from the origin based off of observations made of the tibial plateau. A datum axis that was perpendicular to both the medial/lateral and anterior/posterior datum axes represented the proximal/distal axis. The three datum axes were combined to form the Knee Alignment orientation. The Knee Alignment orientation was aligned with the tibial coordinate system because that was the coordinate system the collected gait data were in (see Section 2.4.1). To apply BCs and loads to the femur the Knee Alignment orientation origin was applied to the knee joint center 
(see Section 2.2.7), while the BCs and loads applied to the tibia used the Knee Alignment orientation with the origin at a centrally located node on the tibial plateau.

\subsubsection{Knee Joint Center}

The knee joint center $(\mathrm{KJC})$ is the center of rotation of the knee, however there is some debate on the exact location of this. The musculoskeletal modeling software OpenSim (National Center for Simulation in Rehabilitation Research, Stanford University), used to analyze dynamic simulations of the human musculoskeletal system [88], defines the $\mathrm{KJC}$ at the midpoint of the femoral epicondyles [89]. This definition seems adequate for the medial/lateral location but does little to describe the anterior/posterior or proximal/distal location. Studies have been performed that measure the sensitivity of joint moments due to choosing different KJC and translation of KJC throughout walking [90], [91], however they still do not provide a definite location of the KJC.

Due to this uncertainty, a trial and error approach was adopted to find the KJC of the FEM. The final KJC was deemed successful because it was the only location that successfully validated the maximum Tibiofemoral (TF) contact pressure for all validation cases (See Section 2.3.1). This point lied on the surface of the femur, in between the femoral epicondyles as defined by OpenSim. It was also chosen because it aligned best with the center of the femoral epicondyles when they were approximated as circles (Figure 11). 

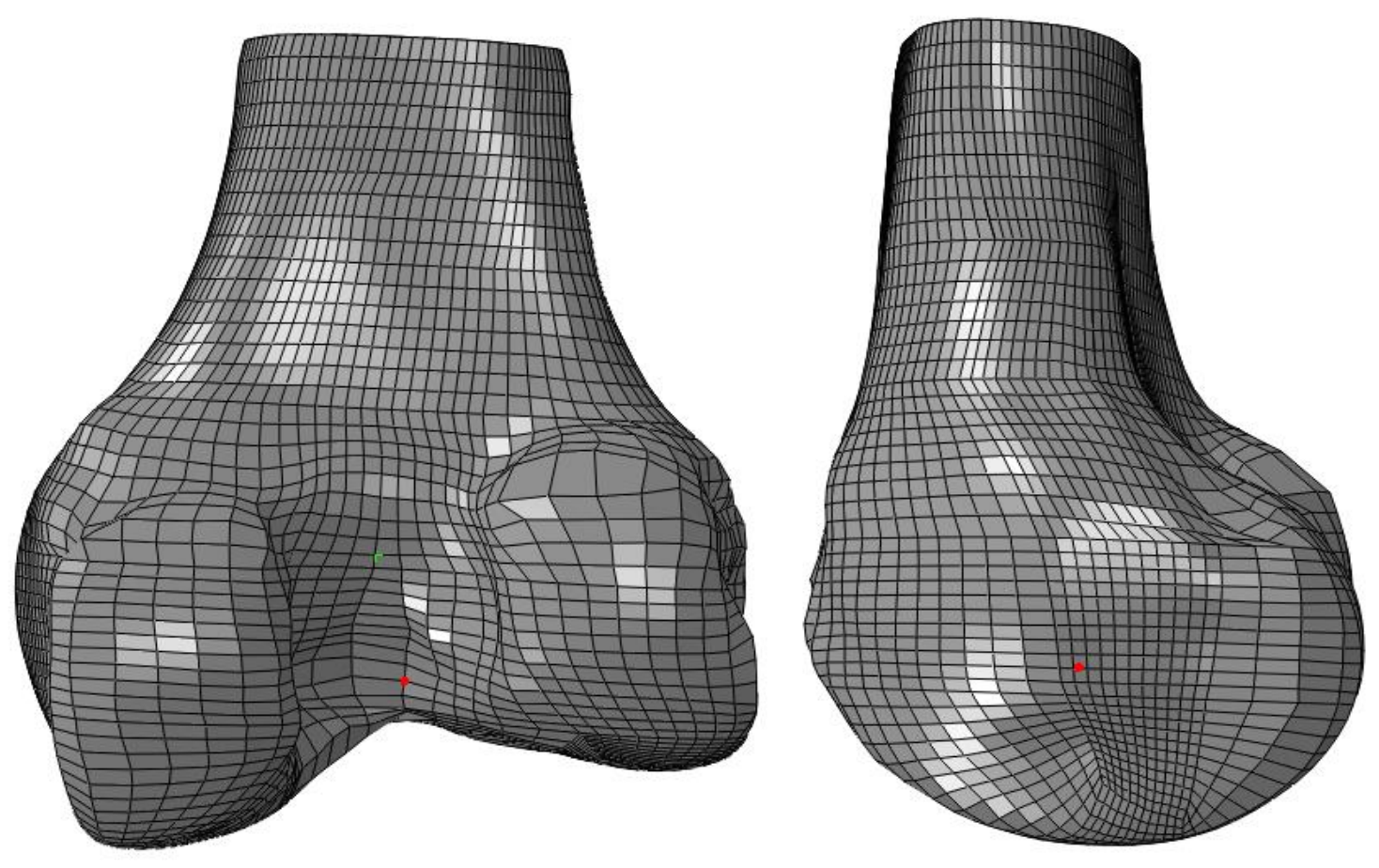

Figure 11: Location of KJC shown in red.

\subsubsection{Automatic Stabilization}

This FEM is highly nonlinear in geometry due to the possibility of buckling in the ligaments and contact issues between the articular cartilage, menisci and ligaments. This nonlinearity can manifest itself as instabilities in the FE solver resulting in an inability to converge to a solution. Abaqus has a built in automatic stabilization scheme to help with these instabilities without having to resort to a dynamic solver or inserting discrete dashpots. There are a few options for automatic stabilization, but the one used in this study was the "specifying a constant damping factor" option. For a detailed description of how Abaqus uses automatic stabilization with specifying a constant damping factor, refer to Appendix A. Care was taken to ensure that the automatic stabilization was not negatively affecting the FE predictions. This was done by measuring the ratio of 
viscous forces $(V F)$ to total forces $(T F)$, checking to see if the viscous forces become dominant in the analysis, in accordance with the Abaqus User's Manual. A damping factor of .0002 was used to validate the ATT of the healthy and AM deficient knee as well as the strain in the AM bundle under anterior load, while a damping factor of .002 was used to validate the articular cartilage contact pressure (see Section 2.3.1). Due to the fact that the primary focus of this study was to examine the changes to articular cartilage loading, the damping factor used to validate articular cartilage contact pressure (.002) was used.

\subsubsection{Mesh Convergence}

A mesh convergence study was performed on the articular cartilage, AM bundle, PL bundle and BPTB graft. The articular cartilage was chosen because accurately analyzing these structures is crucial due to the focus on OA. The ACL bundles and BPTB graft were chosen because it was vital to this study to see the effects of $A C L$ deficient and ACL RS knees during gait. The first step of this convergence study was to create multiple versions, each with a different mesh density, of these structures. The way these structures were meshed in TrueGrid meant the nodes representing the corners of computational blocks did not move regardless of mesh density. These nodes made up a node set for their structures to be used in the analysis portion of the convergence study.

The loading and BCs used were typical knee forces, moments and flexion angles seen during gait [89] applied to the KJC. For the articular cartilage, the nodes in the node set tracked contact pressure as the mesh density increased, while the nodes in the node set for the ACL bundles and BPTB graft tracked the 
maximum principal stress. Figure 12 shows the nodes used in the mesh convergence study. Figure 13 shows the values of sample nodes for some of the structures plotted against the DOFs of said structure. 

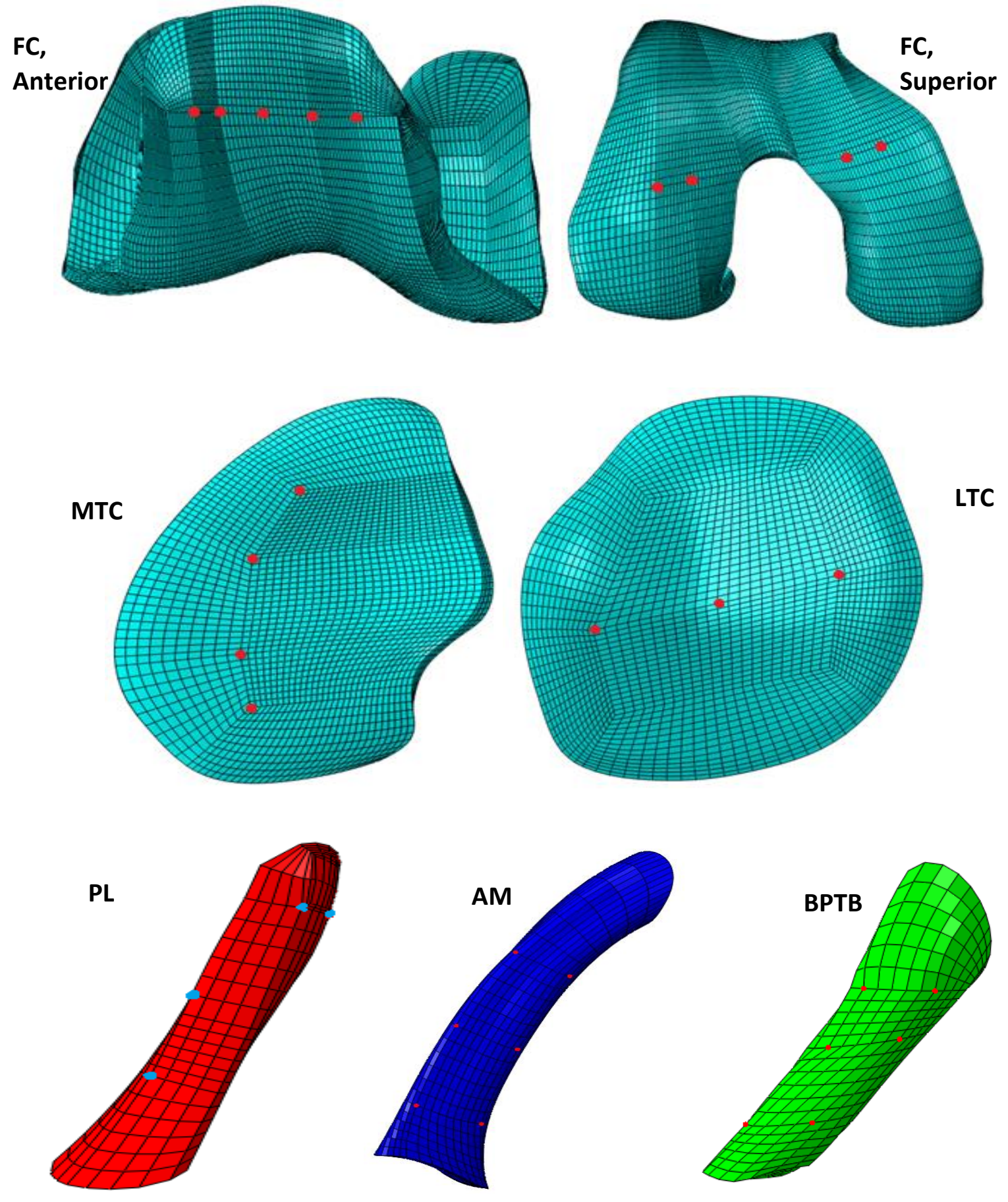

Figure 12: Mesh Nodes of the femoral cartilage (FC), medial tibial cartilage (MTC), lateral tibial cartilage (LTC), PL, AM and BPTB meshed structures used in the Mesh Convergence Study. 

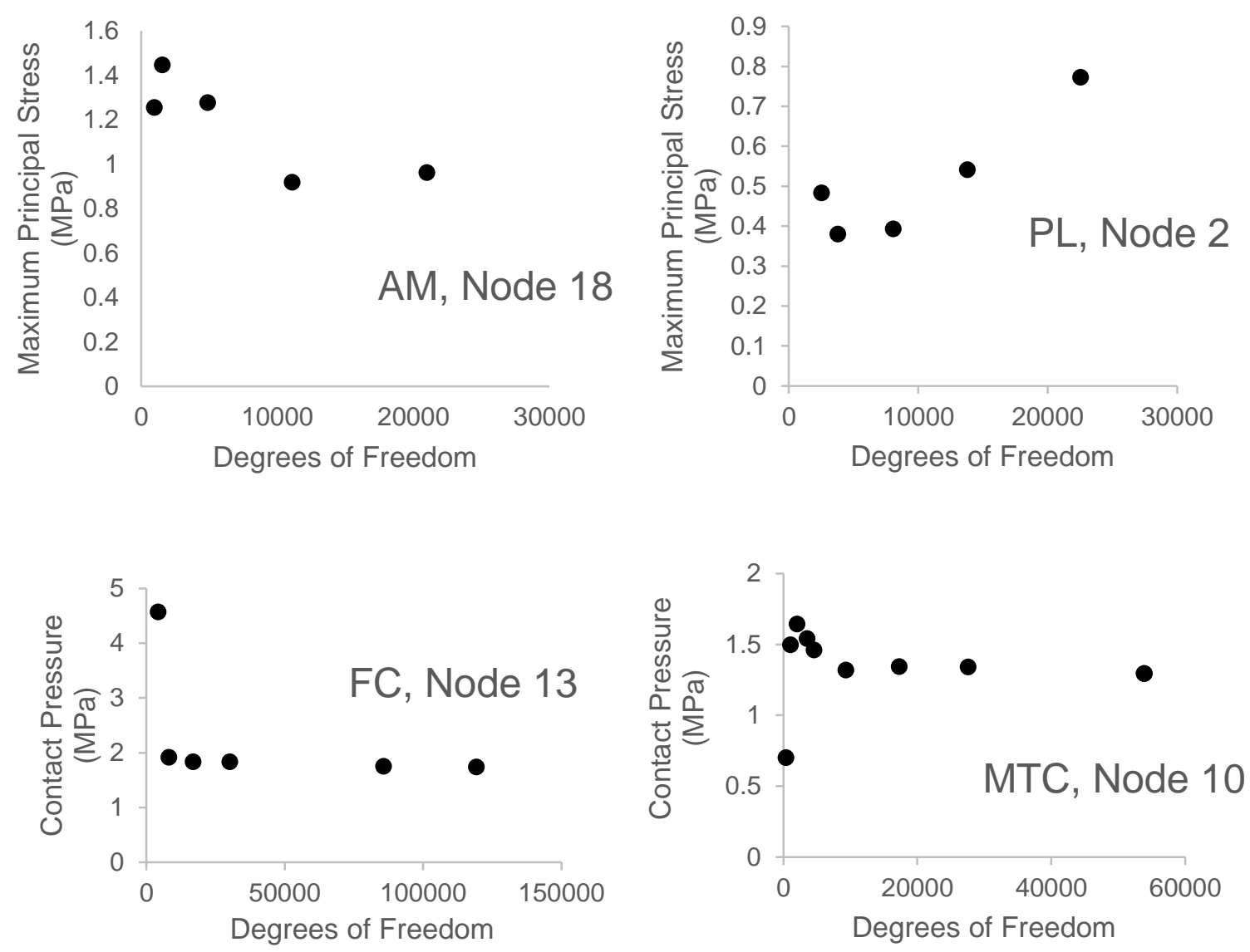

Figure 13: Sample of Convergence study results of the AM bundle, PL bundle, Femoral Cartilage (FC), and Medial tibial Cartilage (MTC).

Due to the fact that each structure is a different size, they are considered converged at different number of DOFs: Femoral cartilage converged at 85785 DOFs and 22368 elements, medial tibial cartilage converged at 27600 DOFs and 6654 elements, lateral tibial cartilage converged at 28476 DOFs and 6948 elements, AM bundle converged at 21000 DOFs and 6264 elements, PL bundle converged at 3813 DOFs and 1024 elements, and the BPTB graft converged at 8352 DOFs and 2415 elements. The converged FEM had 304602 DOFs and 86626 elements. It should be noted that the locations of the nodes used in this 
study are an artifact of the meshing technique used, not necessarily in the location of the predicted peak contact pressure or Von Mises stress. Furthermore, stress concentrations occurred on the articular cartilage due to the sharp edges on the medial and lateral meniscal meshes. For these reasons, output variable readings away from the nodes used in the convergence study should be used with caution. However, because of the good convergence achieved at the nodes used the mesh was considered to be satisfactorily converged.

\subsection{Model Validation}

Validation of a FEM is one of the most important aspects of the development stages because it allows the user to have more trust in the results of a particular study. This model was validated using four human cadaver knee studies: two measuring maximum contact pressure of the medial and lateral tibial cartilage due to a compressive load at various flexion angles [92, 93]; one measuring the ATT for intact ACL and AM deficient knees due to an anterior tibial force [27]; one measuring the strain in the AM bundle due to an anterior tibial force [94].

For each validation study, two steps were created in Abaqus to replicate the experiment: 1) An equilibrium step that attached all the tie constraints, applied the desired flexion angle and allowed the joint to move into its natural position, 2) a loading step that applied the appropriate BCs and loads to the KJC and a node centrally located on the tibial plateau. 


\subsubsection{Maximum Tibiofemoral Contact Pressure}

The first study used to validate the maximum TF contact pressure was performed by Seitz et al. [92]. During the experiment the examiners removed fat, muscle and the Patellofemoral (PF) joint to measure the contact pressure and area of the TF joint underneath the menisci at a flexion angle of $0^{\circ}$ with applied compressive loads of $500 \mathrm{~N}$ and $1000 \mathrm{~N}$. During loading the tibia was fixed, while the femur was allowed to translate in the anterior/posterior and medial/lateral directions as well as rotate internally/externally around its longitudinal axis.

In the equilibrium step the femur was fixed at the $\mathrm{KJC}$ and the tibia was free to move in all six DOFs. In the loading step a concentrated force of $500 \mathrm{~N}$ (1000 N when applicable) was applied to the node stated above on the tibial plateau and all DOFs were fixed except the proximal/distal translation. The femur had the BCs from the experiment applied to the KJC.

The second study used to validate the maximum TF contact pressure was performed by Morimoto et al. [93]. During the experiment the examiners removed the semitendinosus and gracilis tendons to measure the contact pressure and area of the TF joint underneath the menisci with an applied compressive load of $1000 \mathrm{~N}$ at flexion angles of $15^{\circ}$ and $30^{\circ}$ using a pressure film. The flexion angle was set by moving the femur in the coronal plane and locking it in position, while the tibia was allowed to translate in the anterior/posterior and medial/lateral directions as well as rotate internally/externally around its longitudinal axis during loading. 
In the equilibrium step the tibia was free to translate in the anterior/posterior, medial/lateral and proximal/distal directions while the femur had the flexion angle set to $15^{\circ}\left(30^{\circ}\right.$ when applicable) and was free to move in the proximal/distal direction and in the adduction/abduction and internal/external rotations. These BCs were chosen for the equilibrium step because it was felt that they produced the most realistic knee alignment for a given flexion angle. In the loading step the tibia had the BCs from the experiment applied, while the femur was free to move in the proximal/distal direction and rotate in the varus/valgus direction. The loading was done by applying a concentrated load of $1000 \mathrm{~N}$ to the KJC.

\subsubsection{Anterior Tibial Translation}

Due to the fact that restraining ATT is the ACL's primary function, ATT was considered an important parameter to validate the double bundle ACL. An experiment performed by Zantop et al. [27] that measured the ATT due to a 134 $\mathrm{N}$ anterior tibial force of intact $\mathrm{ACL}$ and $\mathrm{AM}$ deficient knees was used to validate the FEM. During the experiment the femur and tibia were potted in a robotic system with six DOFs, similar to other systems used to measure various knee kinematics and in situ forces $[95,96]$. The system fixed the femur in space, fixed the tibia in every DOF except the anterior/posterior direction and applied a $134 \mathrm{~N}$ force to the tibia. The ATT was directly measured from the displacement of the robotic system.

During the equilibrium step the femur was fixed in space while the tibia was free to move in all six DOFs. During the loading step the femur was fixed in 
space while the tibia was allowed to only move in the anterior/posterior direction, with a load of $134 \mathrm{~N}$ applied to the tibia.

\subsubsection{Anteromedial Bundle Strain}

The properties of the AM bundle were validated against a cadaver study performed by Berns et al. [94]. During the study, the experimenters measured the strain of the AM bundle after an anterior load of $140 \mathrm{~N}$ was applied to the tibia of knees with intact ACLs. The femur and tibia were placed in a robotic system similar to the one used by Zantop et al. [27] which had six DOFs and the ability to apply loads in multiple directions. As with the ATT experiment, the femur was fixed in space while the tibia was free to move in the anterior/posterior direction while a load of $140 \mathrm{~N}$ was applied. The strain in the AM bundle was measured using liquid mercury strain gauges (LMSGs).

During the equilibrium step the femur was fixed in space while the tibia was free to move in all six DOFs. During the loading step the tibia was restrained to only move in the anterior/posterior direction, with a load of $140 \mathrm{~N}$ applied to the tibia.

\subsection{Gait Analysis}

A non-compensated gait study was performed using the validated FEM knee joint described above. It was considered non-compensated because gait loads from healthy individuals were applied to FE knee joints modeling ACL deficiencies or ACL RS, when in fact it is well reported that gait changes after ACL injury and RS [97-102]. An ACL deficient compensated gait study could not be performed due to the lack of complete ACL deficient gait data available. 


\subsubsection{Finite Element Gait Analysis}

The non-compensated gait analysis used data collected in collaboration with the CSU Fort Collins Physical Activity Energetic and Mechanics Laboratory (PAL) as outlined in a previous gait analysis [103]. The biomechanics of each participant were quantified using a force measuring treadmill and 10-camera motion capture system that tracked passive reflectors placed on the participant's body. The TF kinematics were calculated using OpenSim's inverse kinematics analysis, while the TF forces were calculated using OpenSim's Joint Reaction analysis. While the action of gait is a continuous motion with continuously changing forces and kinematics, previous studies have modeled discrete points in gait as a quasi-static analysis. The forces and moments were normalized by the average bodyweight of the participants in the study then multiplied by the bodyweight of the FEM subject. As mentioned earlier the functions of the ACL include restraining ATT, excessive axial rotation and maintaining proper varusvalgus alignment and as such the points of gait analyzed were chosen based off of relatively high loading of these functions. The flexion angles, TF forces and moments for the six discrete points used to analyzed gait are shown in Table 9. These forces and moments are reported in a tibial coordinate system applied to the femur. 
Table 9: Knee Joint Forces, Moments and Flexion Angles used in the gait study [103].

\begin{tabular}{|c|c|c|c|c|c|c|}
\hline \multirow{2}{*}{$\begin{array}{c}\text { Knee } \\
\text { Gait }\end{array}$} & $\begin{array}{c}\text { Flexion } \\
\text { Angle (deg) }\end{array}$ & \multicolumn{2}{|l|}{ Joint Reaction Forces (N) } & \multicolumn{2}{l|}{ Joint Reaction Moments (N-mm) } \\
\cline { 4 - 7 } & $\mathbf{F}_{\text {ant }}$ & $\mathbf{F}_{\text {med }}$ & $\mathbf{F}_{\text {comp }}$ & $\mathbf{M}_{\text {add }}$ & $\mathbf{M}_{\text {int }}$ \\
\hline $0 \%$ & 0.75 & -23.41 & 4.27 & 365.07 & -1504.29 & -1292.99 \\
$5 \%$ & 10.5 & -20.9 & 5.8 & 922.14 & -5437.43 & -6706.49 \\
$15 \%$ & 22.84 & -8.94 & 58.86 & 1909.64 & 19197.2 & -2395.34 \\
$30 \%$ & 12.35 & 98.26 & 55.55 & 1020.86 & 10048.1 & -6691.95 \\
$46 \%$ & 5.09 & 104.39 & 167.71 & 2444.65 & 14278.7 & -13095.3 \\
$60 \%$ & 41.75 & 51.37 & -22.67 & 351.15 & -8286.86 & 976.05 \\
\hline
\end{tabular}

As with the validation studies two steps were created in Abaqus to mimic gait: 1) An equilibrium step that applied all the tie constraints and flexion angle to the knee joint, 2) a loading step that applied the forces and moments to the KJC and BCs to the tibia and femur. During the equilibrium step the same BCs applied during the Morimoto at al. [93] validation were applied to the tibia and femur: the tibia was free to translate in the anterior/posterior, medial/lateral and proximal/distal directions; the femur had the appropriate knee flexion angles applied to the $\mathrm{KJC}$ and was free to move in the proximal/distal direction and in the adduction/abduction and internal/external rotations. During the loading step the tibia was fixed in place while the femur was only restrained in the flexion/extension angle. 
It should be noted that the forces and moments applied to the femur do not include any forces or moments generated by the PF joint. The OpenSim model uses the PF joint for kinematic constraints on the TF joint, however the numbers output are strictly forces and moments from the TF joint. To estimate the error associated with excluding the PF joint forces, the appropriate contact forces of the PF joint measured by Lin et al. [104] during gait were applied to the femur at the suspected PF contact location at a specific point of gait. The resulting maximum articular cartilage contact pressure $(\mathrm{CP})$ and contact area (CA) were compared to the same gait point without the PF contact force.

\subsubsection{Output Variables}

When using Abaqus, specific variables of interest must be declared before an analysis is performed. The output variables used for the gait analysis included contact pressure (CPRESS), contact area (CAREA), displacement components $(U)$, viscous forces $(V F)$ and total forces (TF). As mentioned in Section 2.2.8, the ratio of viscous forces, $V F$, to total forces, $T F$, were used to make sure the automatic stabilization was not negatively affecting the predicted results. The contact pressure, contact area and contact forces have previously been used for gait analysis and are good indicators of the progression of OA [34-36, 40, 41]. As with the validation studies, the maximum contact pressure was measured by selecting and averaging a group of nodes roughly the size of the pressure pad used by Seitz et al. surrounding the peak node. Due to the fact that the primary role of the ACL is ATT prevention, anterior displacement was chosen as another 
important parameter to track for the various levels of $A C L$ deficiency and $A C L$ RS. 


\section{CHAPTER 3 RESULTS}

\subsection{Model Validation Results}

As mentioned earlier, the FEM was validated using four cadaver studies: Two measuring the maximum TF contact pressure at various flexion angles and applied loads [92, 93], one measuring the ATT due to an applied anterior tibial load of a knee with an intact ACL and a knee that was AM deficient [27], and one measuring the strain in the AM bundle due to an applied anterior tibial load [94].

\subsubsection{Maximum Tibiofemoral Contact Pressure}

The maximum contact pressure of the modeled Seitz et al. [41] experiment was measured using the output variable CPRESS. This was done by selecting a group of nodes around the maximum contact pressure node that represented a similar surface area to the resolution of the measurement devices

used in the experiments, $1.6 \mathrm{~mm}^{2}$, and averaging the contact pressure of said nodes. For all cases the maximum pressure was determined in both the medial and lateral tibial cartilage. Figure 14 shows the contact pressure map of the medial and lateral tibial cartilage for both loading cases of the Seitz et al. experiment. 


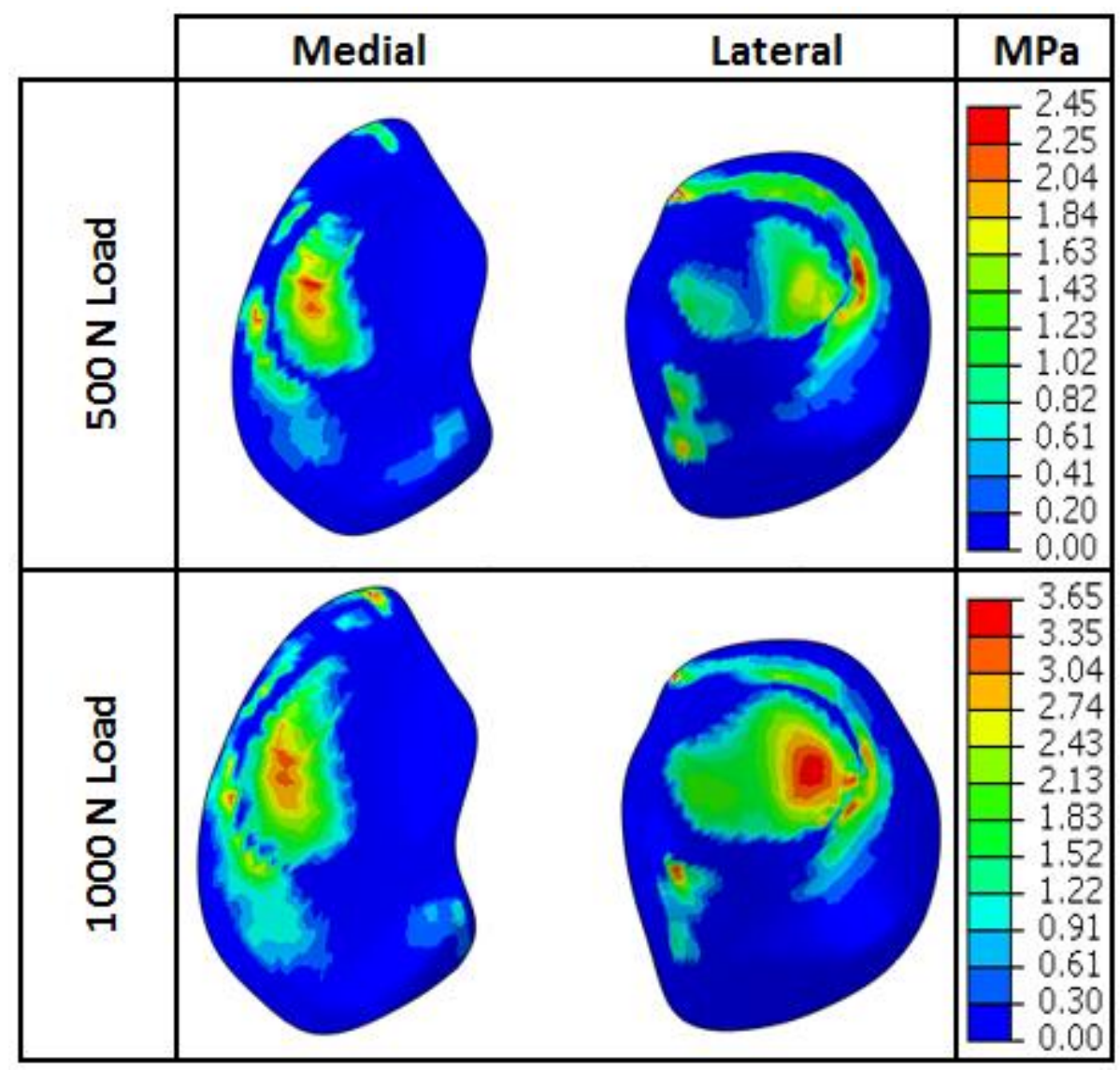

Figure 14: Contact Pressure of the medial and lateral tibial cartilage during loading at full extension with an intact ACL. 

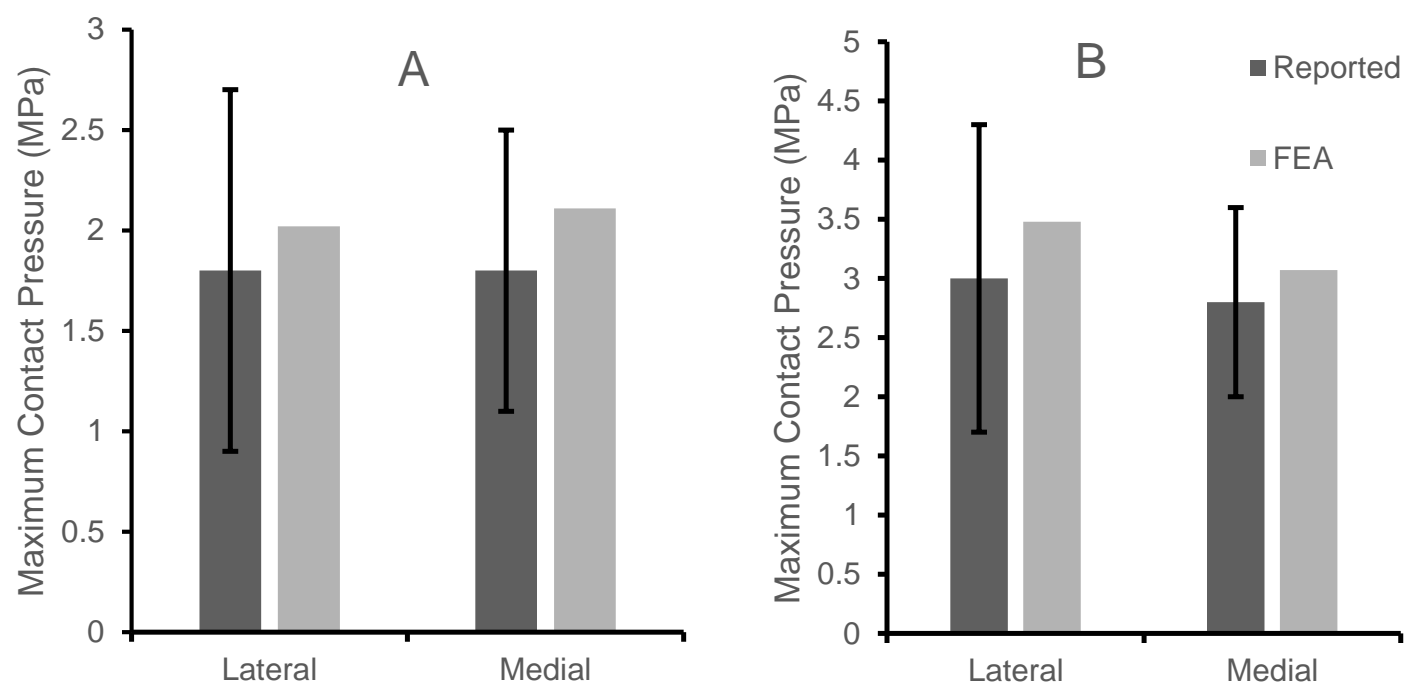

Figure 15: FEA Validation vs Experimental Results of CP during loading with an intact ACL. A) $500 \mathrm{~N}$ Load. B) $1000 \mathrm{~N}$ Load [41].

Figure 15 clearly shows that FEA results for both the $500 \mathrm{~N}$ and $1000 \mathrm{~N}$ loading falls within one standard deviation of the reported average maximum contact pressure. It should be noted that stress concentrations under the lateral anterior meniscal horn, for the $500 \mathrm{~N}$ case, and the medial anterior meniscal horn, for the $1000 \mathrm{~N}$ case, were above one standard deviation from the mean. However, as mentioned in Section 2.2.9 these areas would be analyzed with caution, possibly ignored as in this case, due to sharp edges present on all the meniscal horns. With these results, it was deemed that the FEM was successfully validated at full knee extension during these loading cases.

As with the first validation study, a group of nodes around the peak node, surface area roughly $1.6 \mathrm{~mm}^{2}$, were averaged to determine the maximum pressure of the medial and lateral tibial cartilage. Figure 16 shows the contact 
pressure map of the medial and lateral tibial cartilage for both loading cases of the Morimoto et al. experiment [42].

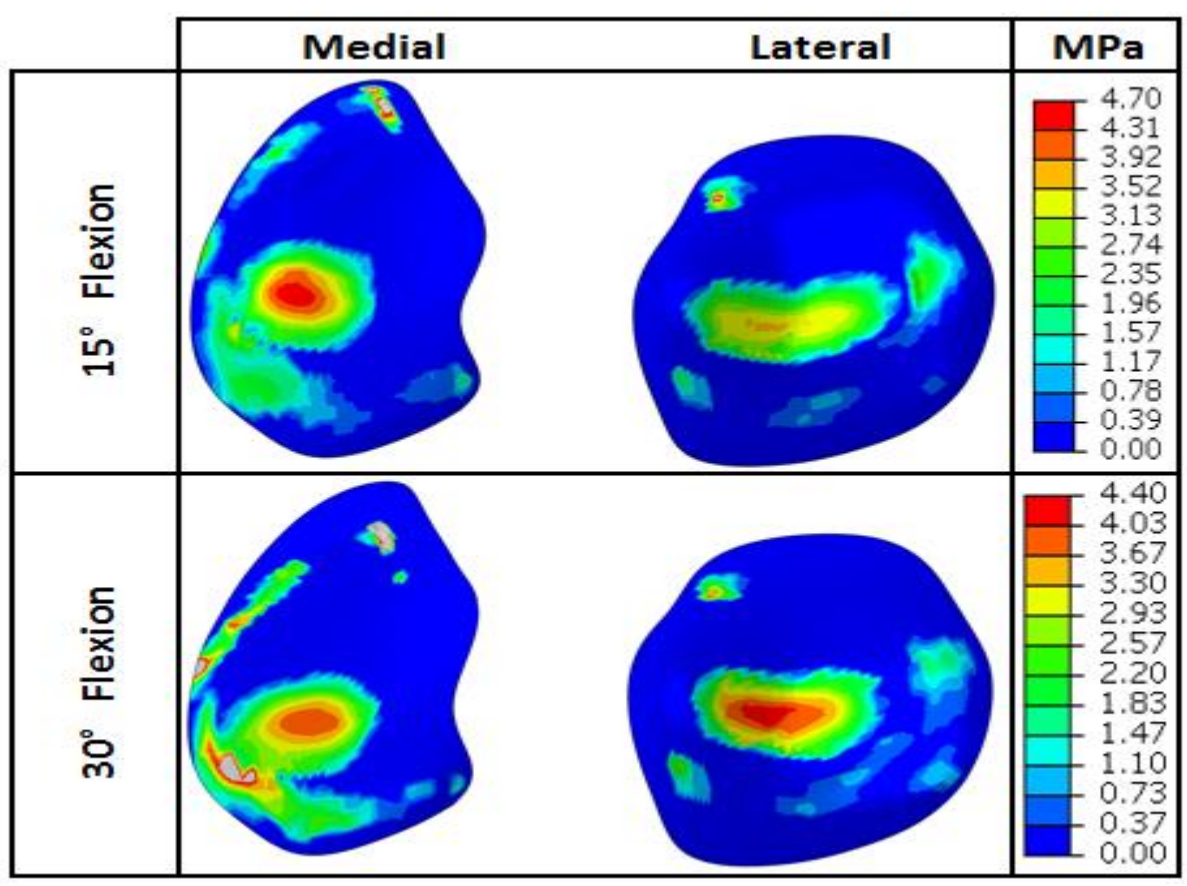

Figure 16: Medial and lateral tibial cartilage contact pressure during loading at $15^{\circ}$ and $30^{\circ}$ with an intact $\mathrm{ACL}$.
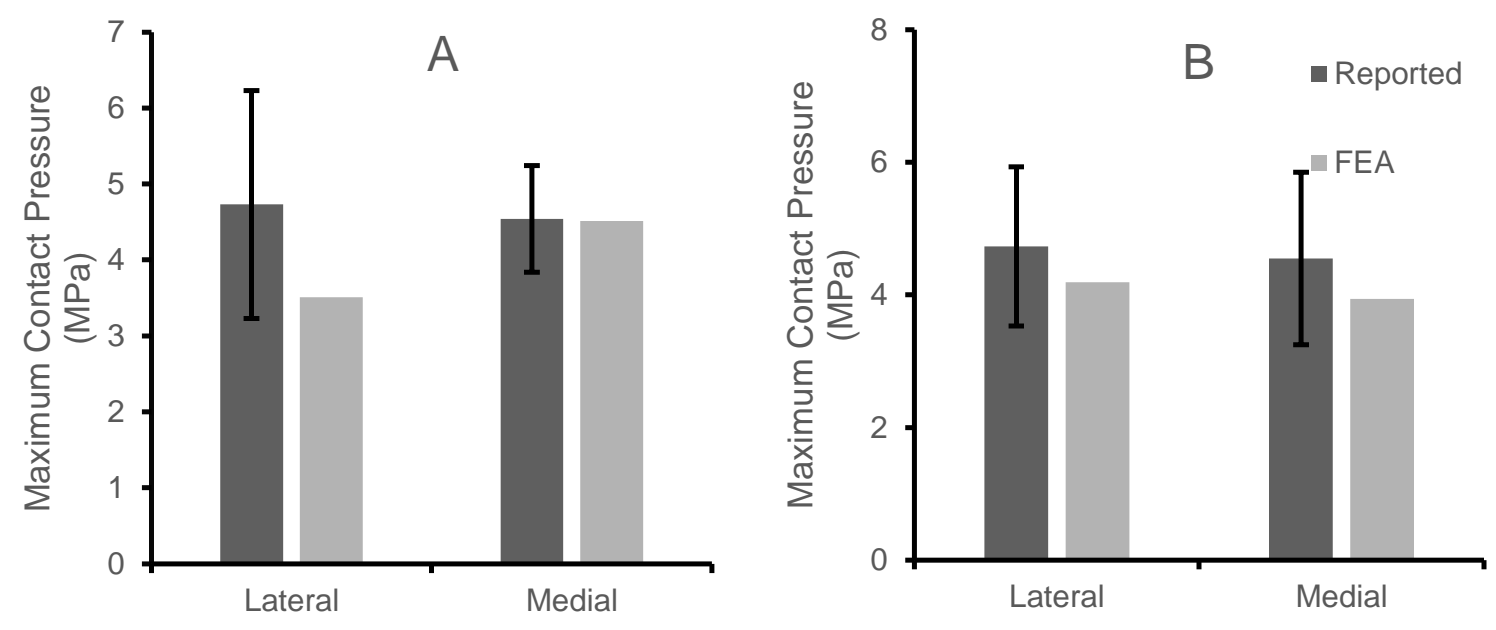

Figure 17: FEA Validation vs. Experimental Results of CP during loading with an intact ACL. A Loading at $15^{\circ}$ Flexion. B Loading at $30^{\circ}$ Flexion [42]. 
The FEM is considered validated for loading at various knee flexion angles, with results falling within one standard deviation of the reported average (Figure 17). It should be noted that stress concentrations under the medial meniscus anterior horn, for both angles, and the inner circumference edge of the medial meniscus, for $30^{\circ}$ loading, were ignored. However, when these stress concentration nodes were averaged with the surrounding nodes they were within one standard deviation of the reported mean. They were ignored because it was felt that they did not accurately represent interaction between the medial tibial cartilage and medial meniscus.

It should be noted that these experiments were also performed at different loading conditions: Seitz et al. [92] loaded the cadaver knee at $30^{\circ}$ with $500 \mathrm{~N}$ and $1000 \mathrm{~N}$ compressive forces, Morimoto et al. [93] loaded the cadaver knee at $0^{\circ}$ with a $1000 \mathrm{~N}$ compressive force. However, when these loading conditions were repeated with the FEM, all the predicted results were more than one standard deviation from the reported measurements. This inability to validate these experiments is an issue that should be looked into during future work, yet there may be some explanation for these issues. When both experimenters loaded the knee at $0^{\circ}$ and $30^{\circ}$ with $1000 \mathrm{~N}$ none of the reported maximum contact pressures are within one standard deviation of the other's reported value. 

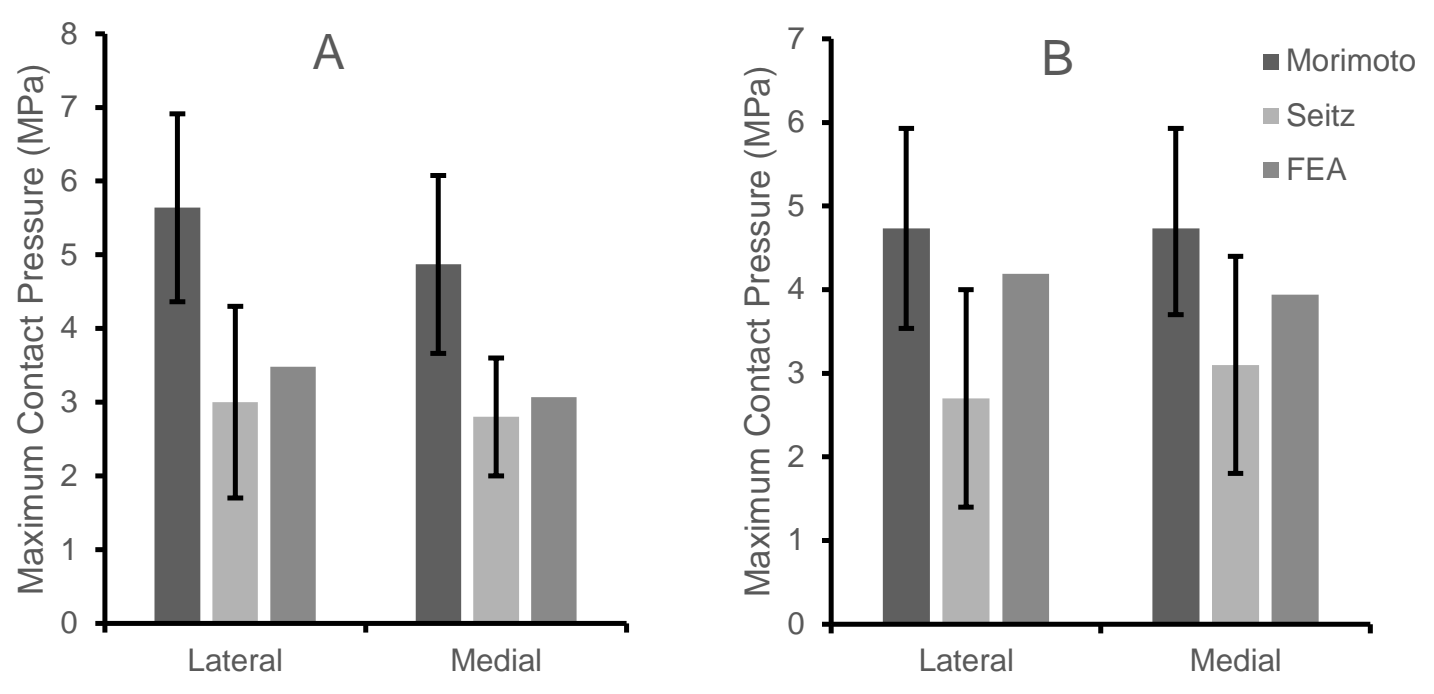

Figure 18: Morimoto, Seitz and FEA results with $1000 \mathrm{~N}$ compressive load at $\mathrm{A}$ $0^{\circ}$ and $\mathrm{B} 30^{\circ}[41,42]$ with intact $\mathrm{ACL}$.

These discrepancies in maximum contact pressure could be an artifact of loading the femur versus the tibia or using different equipment to measure the pressure: Seitz et al. used a thin, flexible pressure sensor (K-Scan Type 4000, 2 x $920.7 \mathrm{~mm}^{2}$, 9,000 psi; Tekscan, Inc., South Boston, MA) [92], while Morimoto et al. used low-pressure measurement films (pressure range, 2.5 to $10 \mathrm{MPa}$, Fuji Prescale Film; Fujifilm, Valhalla, NY) [93]. Due to these discrepancies in the reported maximum contact pressure and the ability of the FEM to validate with at least one of the loading conditions for all the reported flexion angles, the FEM was considered validated for loading at various flexion angles.

\subsubsection{Anterior Tibial Translation}

The ATT was measured from displacement output variable $U$. Due to the BCs applied to the tibia, all the tibial nodes had the same displacement so no averaging was needed. The predicted and reported ATT are shown in Figure 19. 


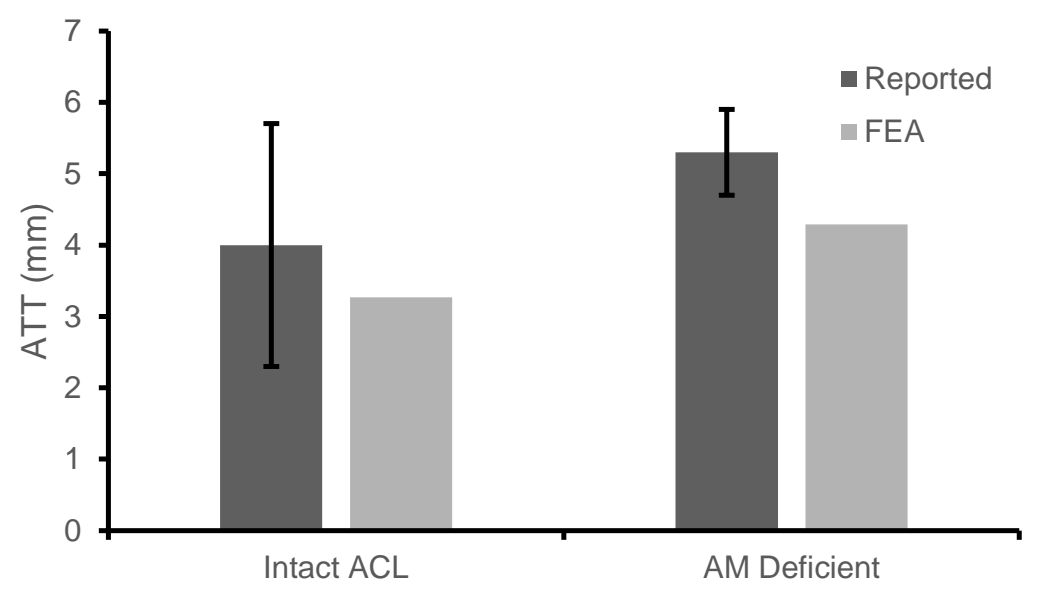

Figure 19: FEA Validation vs Experimental Results of ATT [27].

The predicted ATT of the FEM with an intact ACL falls within one standard deviation of the reported mean ATT while the predicted ATT of the FEM with an AM deficiency is only within two standard deviations of the mean reported ATT. The result of the intact ACL ATT was interpreted as validation of the interaction between and characteristics of the two bundles. Despite the fact that the predicted ATT of the AM deficient FEM was outside one standard deviation of the mean, it was still considered a validation of the properties of the PL bundle because the predicted trend of ATT increase from intact ACL to AM deficient followed the trend reported: experimental results showed an increase of $32.5 \%$; FEM predictions showed an increase of $31.2 \%$.

It should be noted that the experimenters also performed this loading condition with a PL deficient knee. However, when this case was repeated with the FEM the predicted ATT was more than one standard deviation below the average and the predicted trend of ATT increase from intact ACL to PL deficient did not match the reported trend. The disagreement of predicted ATT and 
reported ATT could be explained by the fact that the knees tested were much older (age range, 53-71 [27]) than the knee being modeled. A study comparing young ACLs to older ACLs showed a stiffness reduction of $29 \%$ in older ACLs [75]. Another possible factor in the discrepancy between predicted and reported ATT is that Zantop et al. [39] don't report what percent of knees being tested are male or female knees. Another study showed that male ACLs are $54 \%$ more stiff when compared to female ACLs [76]. Both of these factors explain could why the predicted ATT of PL deficient knees does not agree with the reported ATT; they also explain why the predicted AM deficient ATT is more than one standard deviation below the experimental ATT.

\subsubsection{Anteromedial Bundle Strain}

The strain of the AM bundle was measured by measuring the length of the AM bundle before and after the applied load. Due to the fact that the location of the LMSGs was not specifically mentioned, the strain of the AM bundle was measured at a few locations and averaged together, shown in Figure 20. 


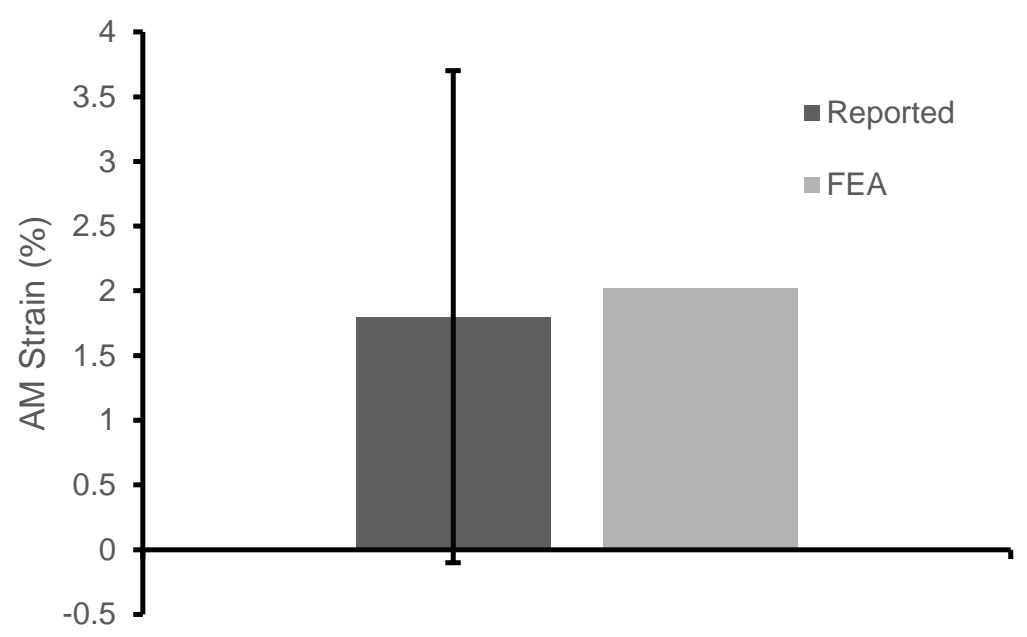

Figure 20: FEA Validation vs. Experimental Results of AM Bundle Strain under $140 \mathrm{~N}$ tibial anterior load [94].

The predicted strain of the AM bundle due to an anterior tibial load of 140 $\mathrm{N}$ was within one standard deviation of the reported mean AM bundle strain. This result was considered a validation of the AM bundle properties.

\subsection{Gait Results}

The results of the $\mathrm{CP}, \mathrm{CA}, \mathrm{ATT}$ and viscous force to total force ratio for all ACL cases are tabulated in Appendix B. In general, when the ACL was modeled with an AM bundle injury, PL bundle injury or complete ACL injury there was very little change to either the $\mathrm{CP}$ or $\mathrm{CA}$. The largest percent change of either variable was a $14.3 \%$ increase of the CP of the medial tibial cartilage when modeling $60 \%$ of gait with an injured AM bundle. However, the majority of these predicted results showed less than a $1 \%$ change compared to intact ACLs for all points of gait. These results are not surprising due to the fact that the material properties of the injured AM and PL bundle are close to lower values of reported healthy 
ligament properties [45]. For this reason, further discussion of predicted results will only consider intact $A C L, A M$ deficient, PL deficient, ACL rupture and BPTB graft knees.

When looking at the predicted results of a healthy knee, it is clear that throughout gait whichever compartment of the knee, medial or lateral, supporting the majority of the load can shift to the other compartment. Due to this shift, there is no one point of gait that has the largest percent increase of CP or CA for all articular cartilage for a given ACL deficiency or RS. To coincide with this fact, the reported predicted results are only shown when the articular cartilage surface is most at risk of the development of OA.

The largest $\mathrm{CP}$ predicted, $9.37 \mathrm{MPa}$, was on the medial portion of the femoral cartilage during $46 \%$ of gait in a knee with a ruptured ACL. Looking at the largest CP is a good indication of what part of the knee supports the most load, however for this study a more pertinent analysis would be to observe the largest percent increase in $\mathrm{CP}$ in $\mathrm{ACL}$ deficiencies and $\mathrm{RS}$ compared to intact ACLs. Knees with a predicted increase in CP are considered more at risk of the development of OA. The largest percent change of CP from an intact ACL knee was a $27.9 \%$ increase in the medial tibial cartilage at $60 \%$ gait of an AM deficient knee. The maximum increase of $\mathrm{CP}$ in each cartilage structure for all of gait and their representative $\mathrm{CP}$ contour plots are shown below. 


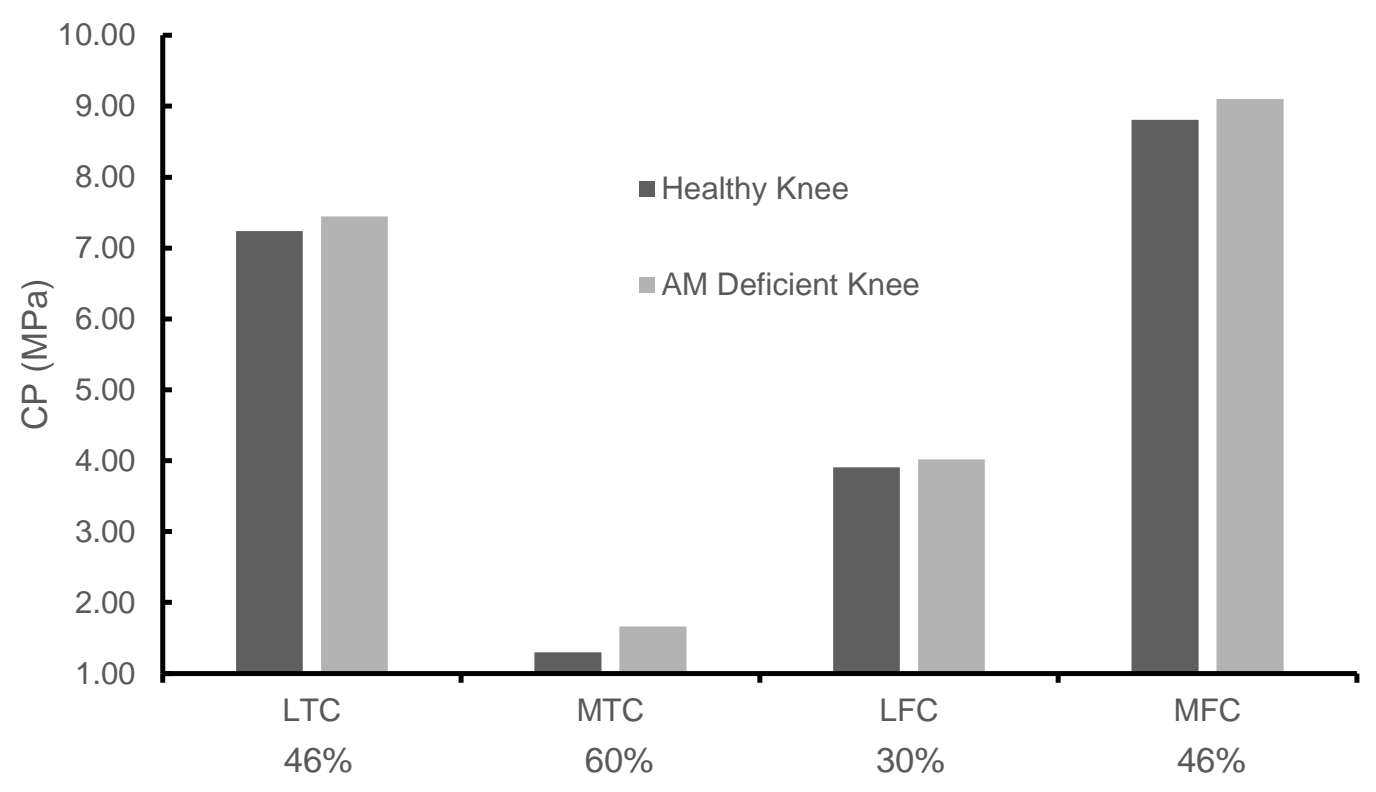

Figure 21: The point of Gait where the largest percent increase of CP occurred for each articular cartilage between intact $A C L$ and $A M$ deficient Knees. 


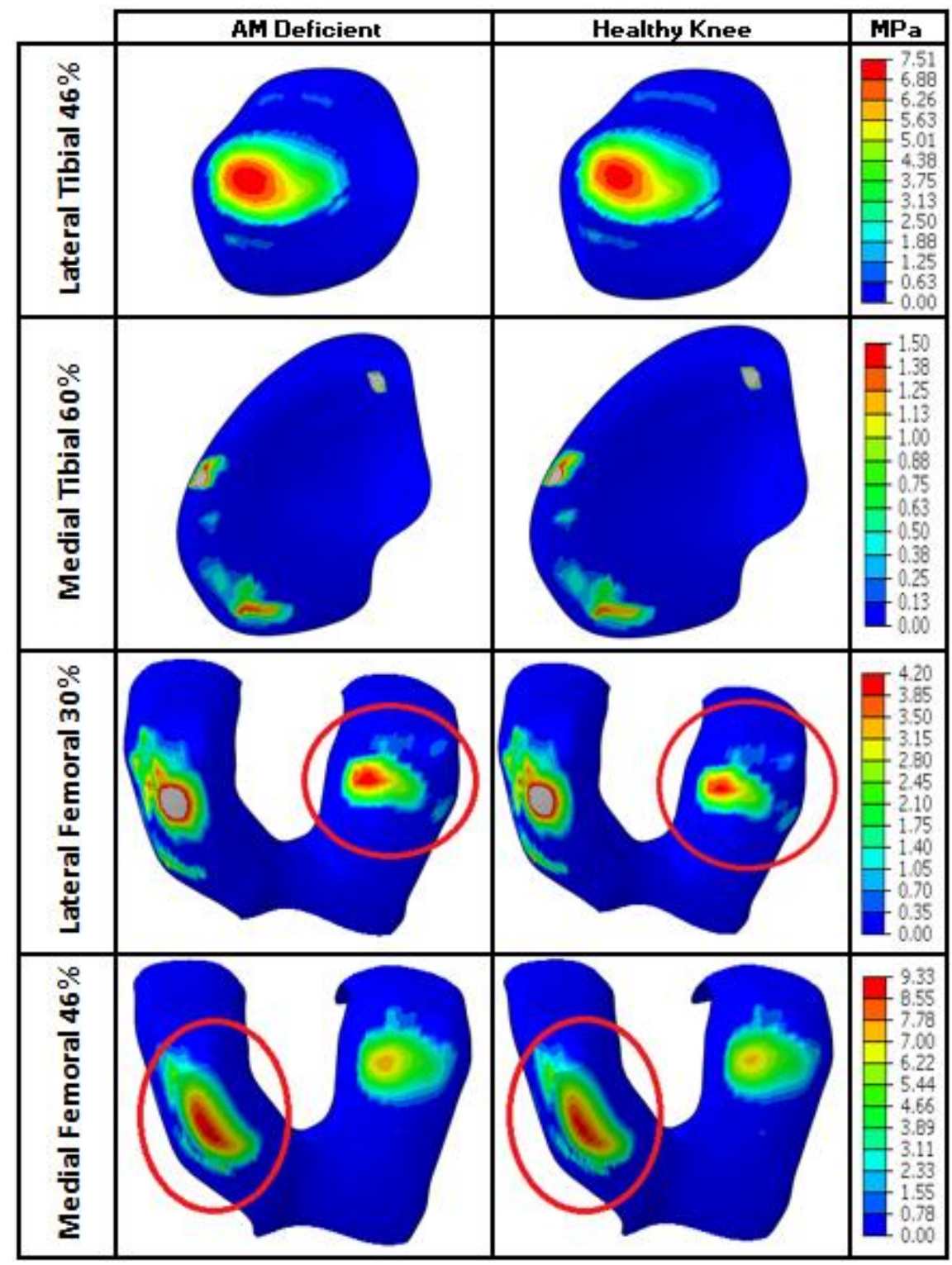

Figure 22: Contour CP plot of each articular cartilage at the point of Gait when there was the largest percent increase of maximum increase of CP between intact $A C L$ and $A M$ deficient Knees. 


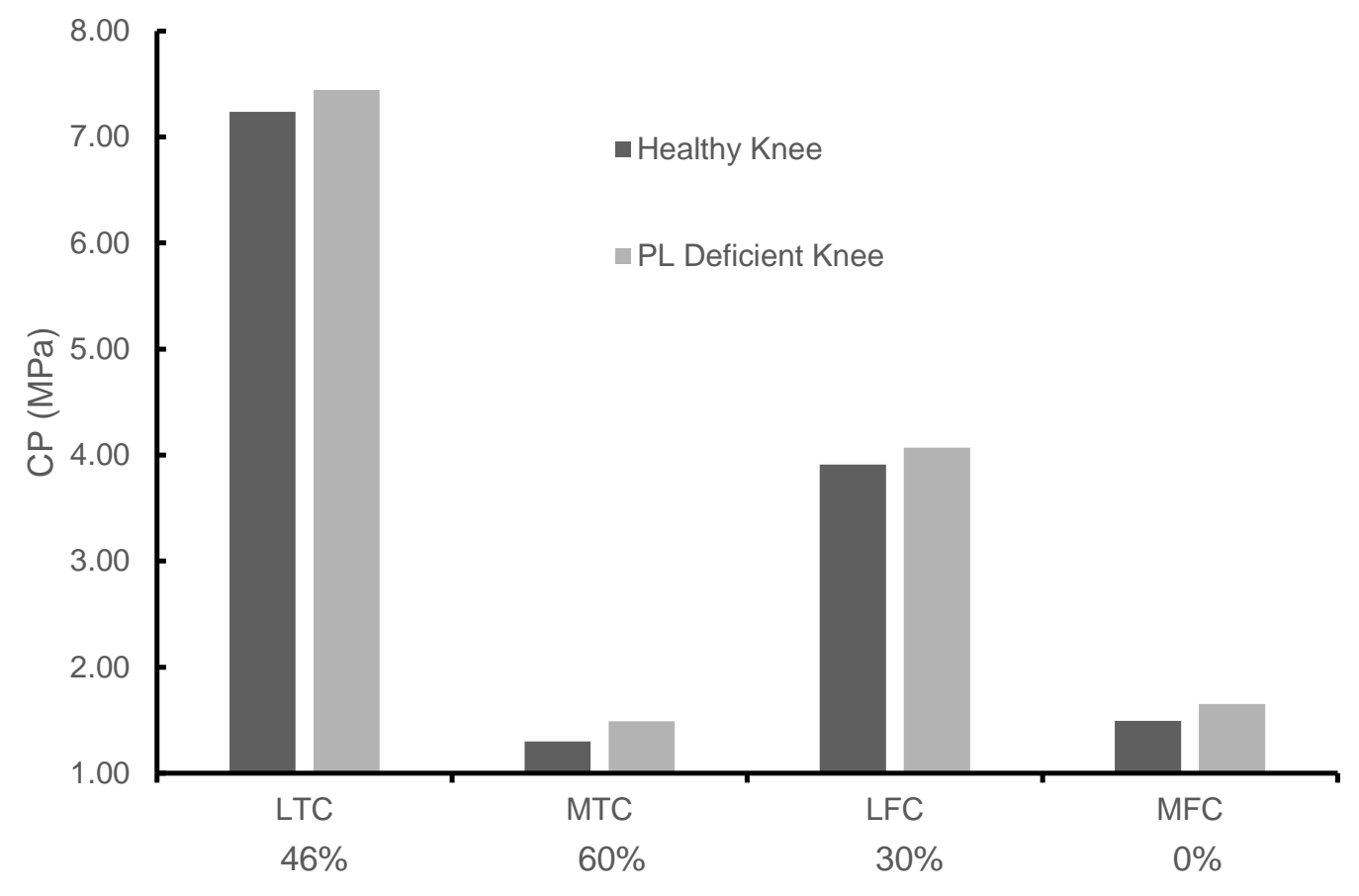

Figure 23: The point of Gait where the largest percent increase of CP occurred for each articular cartilage between intact $A C L$ and PL deficient Knees. 


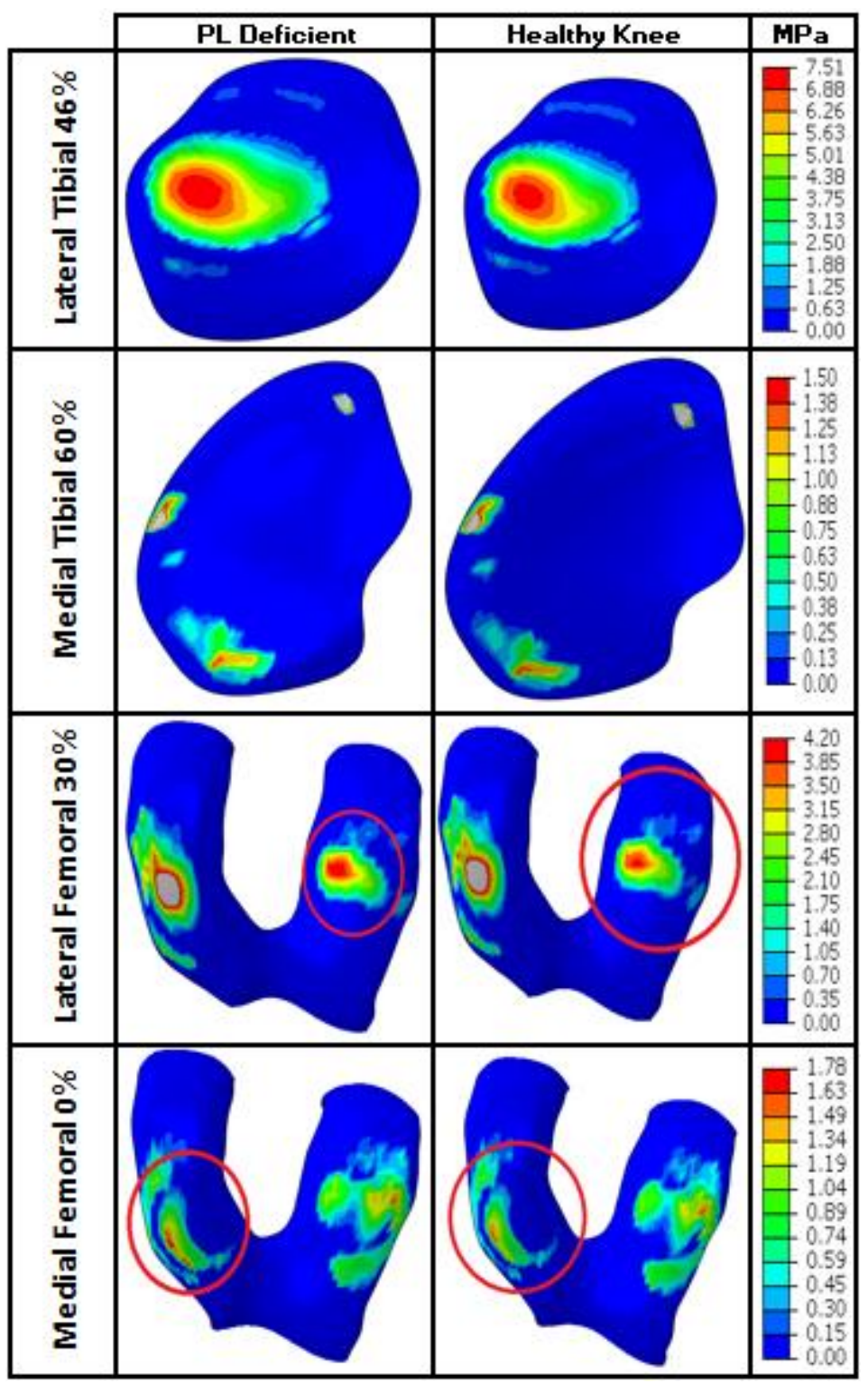

Figure 24: Contour CP plot of each articular cartilage at the point of Gait when there was the largest percent increase of maximum increase of CP between intact ACL and AM deficient Knees. 


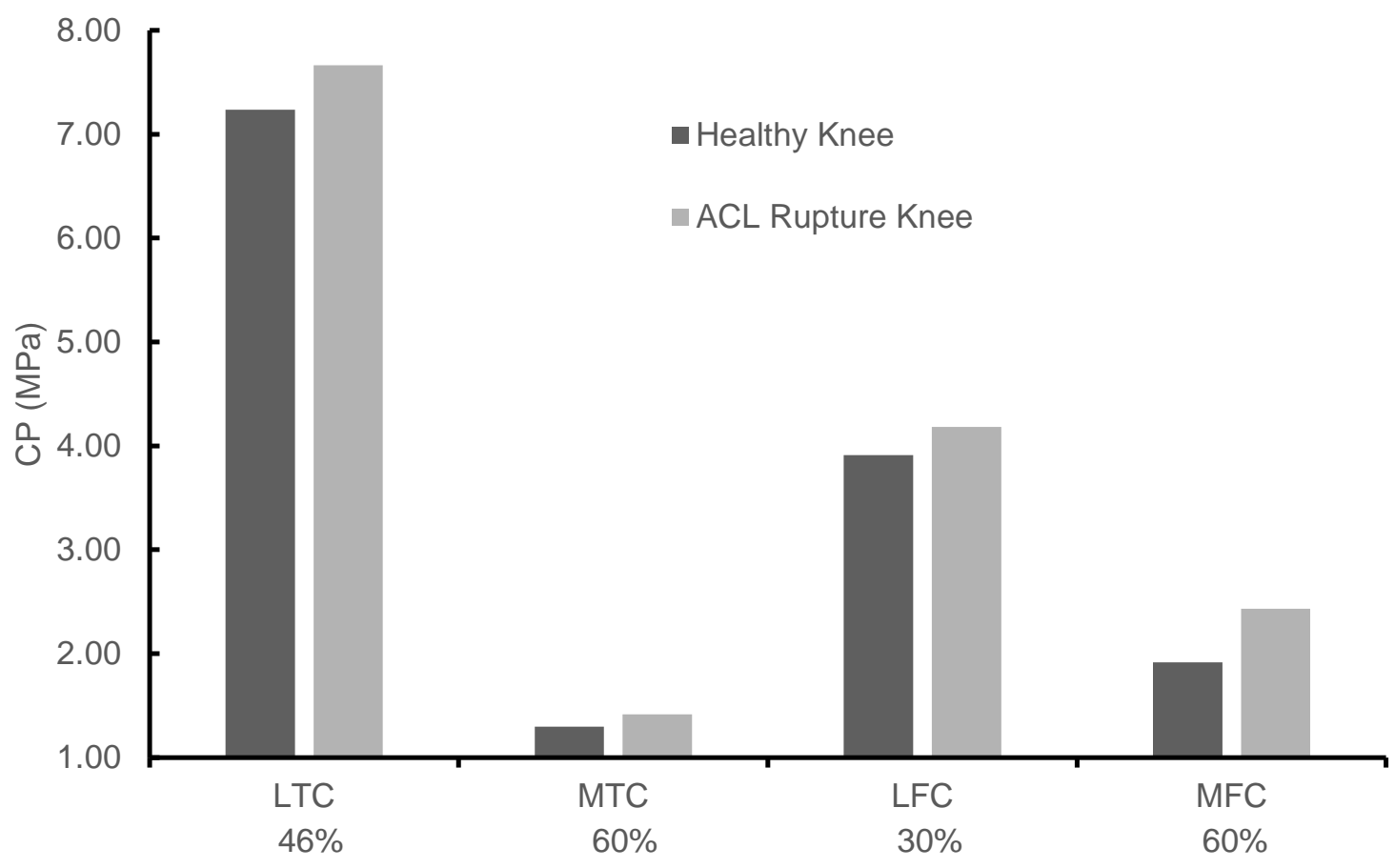

Figure 25: The point of Gait where the largest percent increase of CP occurred for each articular cartilage between intact $A C L$ and $A C L$ ruptured Knees. 


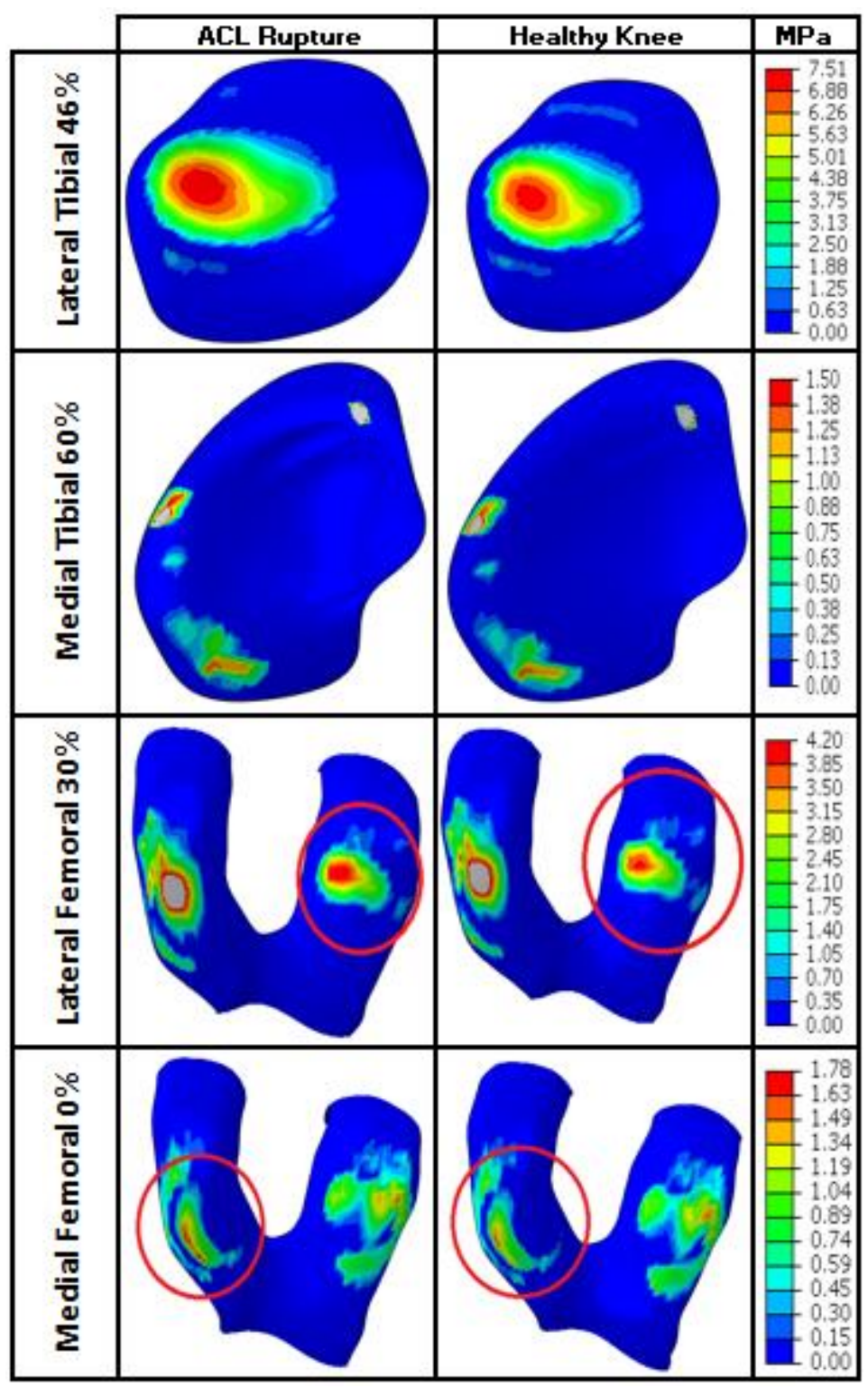

Figure 26: Contour CP plot of each articular cartilage at the point of Gait when there was the largest percent increase of maximum increase of CP between intact $A C L$ and $A C L$ ruptured Knees. 


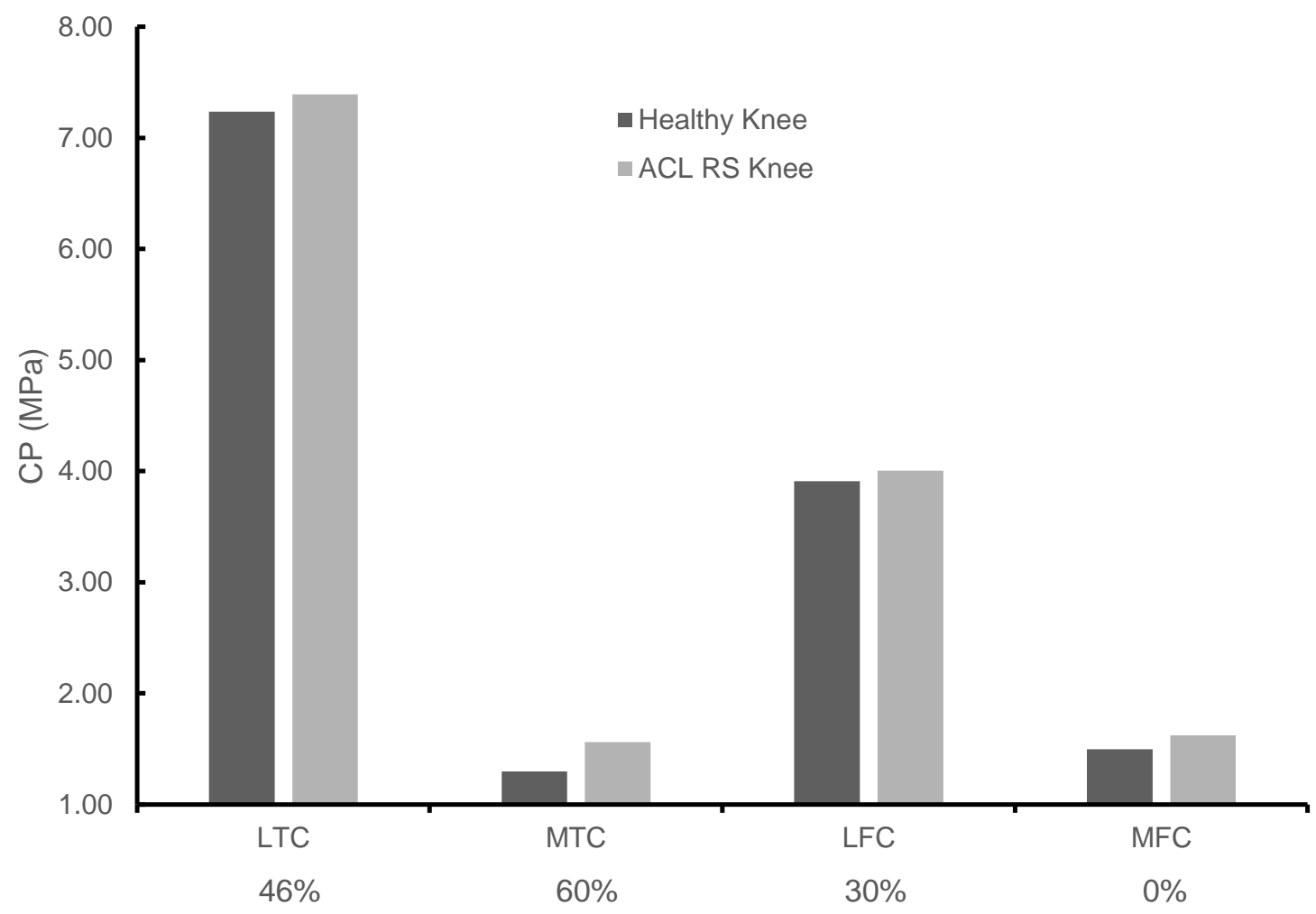

Figure 27: The point of Gait where the largest percent increase of CP occurred for each articular cartilage between intact ACL and ACL RS Knees. 


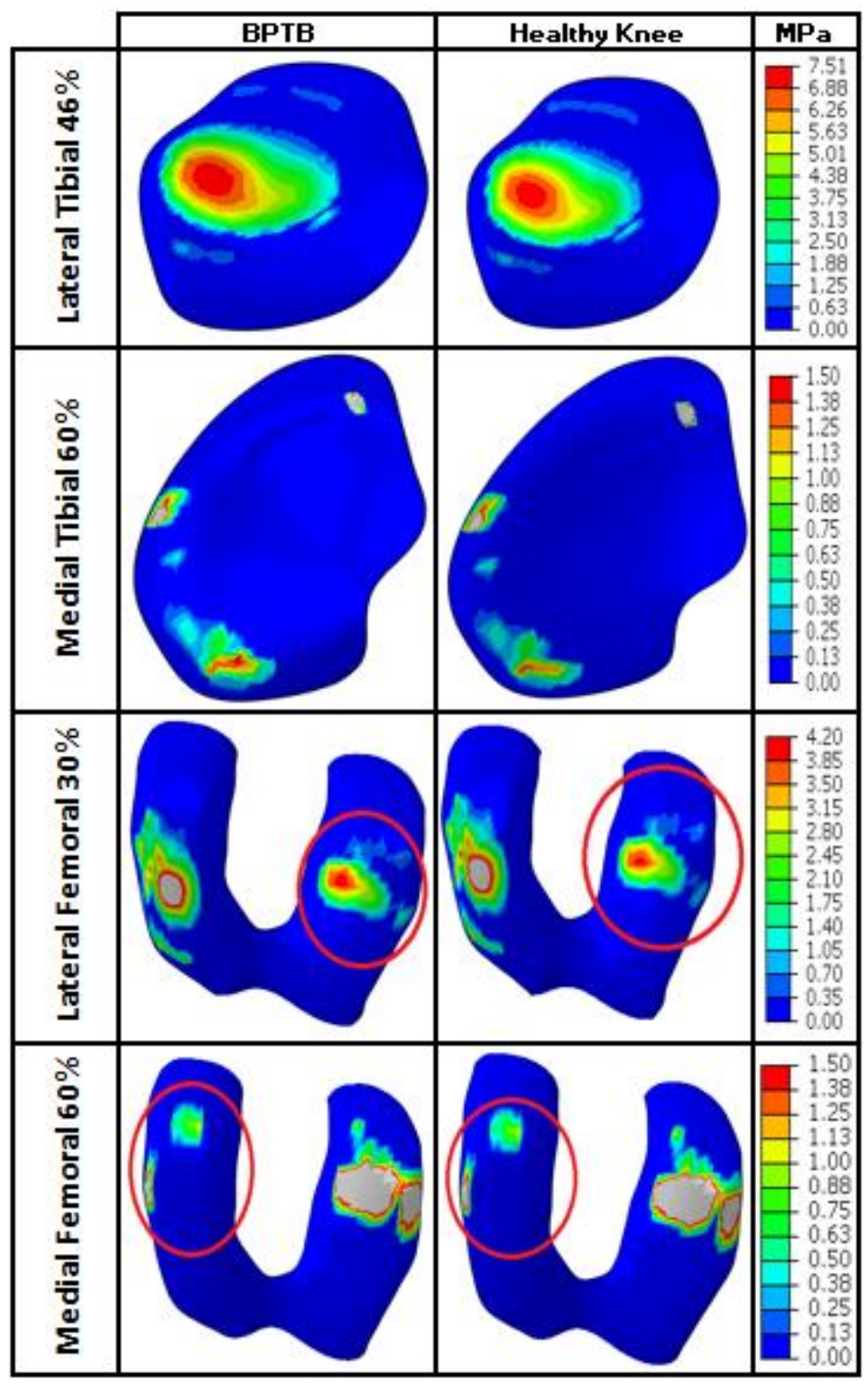

Figure 28: Contour CP plot of each articular cartilage at the point of Gait when there was the largest percent increase of maximum increase of CP between intact ACL and ACL RS Knees.

The largest CA predicted, $392.29 \mathrm{~mm}^{2}$, was on the lateral tibial cartilage during $5 \%$ of gait in an AM deficient knee. When comparing the predicted results of damaged to intact ACLs, knees with an increase of CA are considered more at risk of the development of $\mathrm{OA}$. The largest percent change of articular cartilage CA from an intact ACL knee was an $11.9 \%$ increase in the medial portion of the 
femoral cartilage at $60 \%$ of gait of a complete ACL ruptured knee. For all points of gait modeled with a PL bundle injury, the CA of the lateral tibial cartilage was smaller than the CA predicted with an intact $A C L$ so no results were graphed.

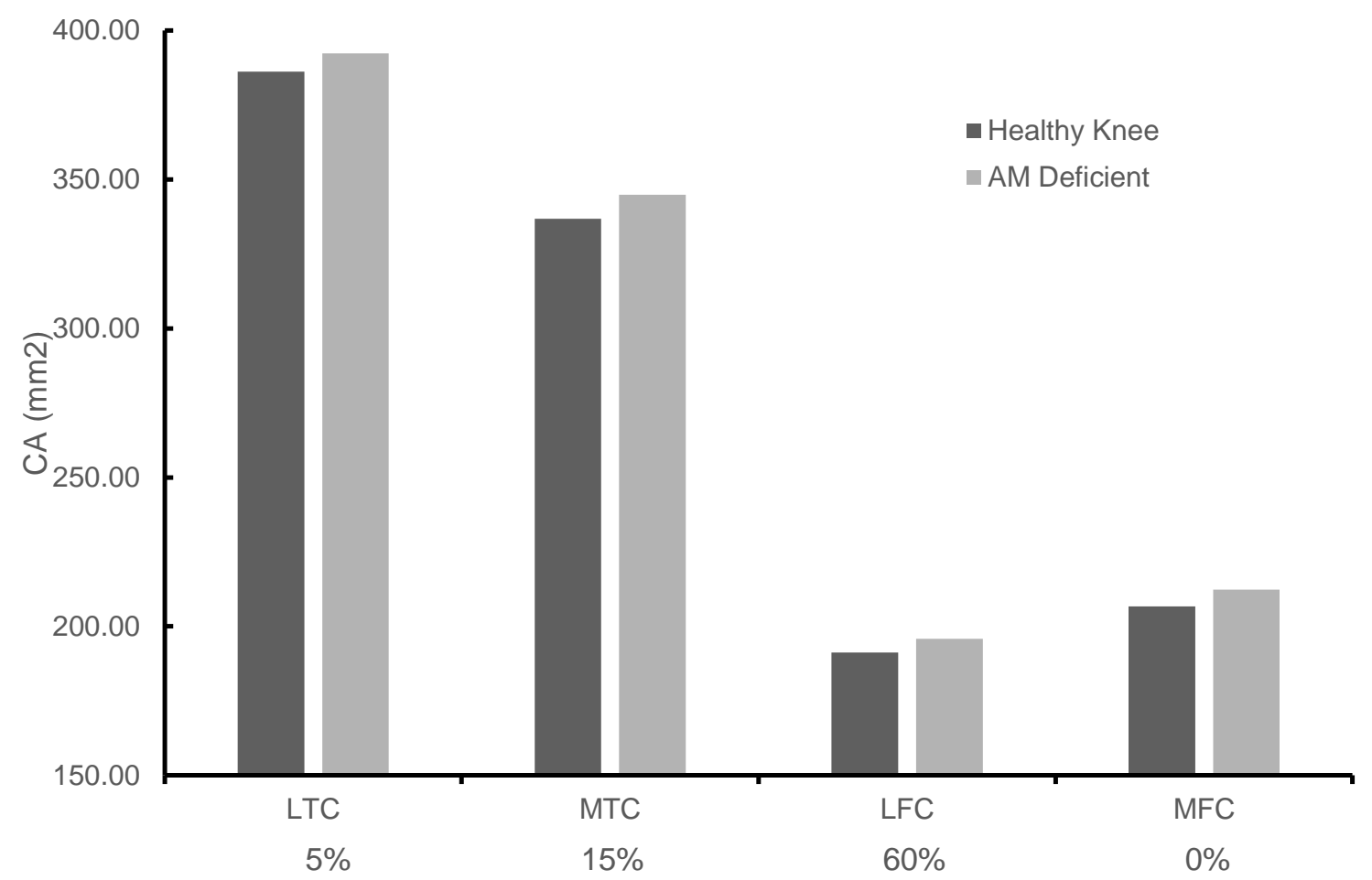

Figure 29: The point of Gait where the largest percent increase of CA occurred for each articular cartilage between intact $A C L$ and $A M$ deficient Knees. 


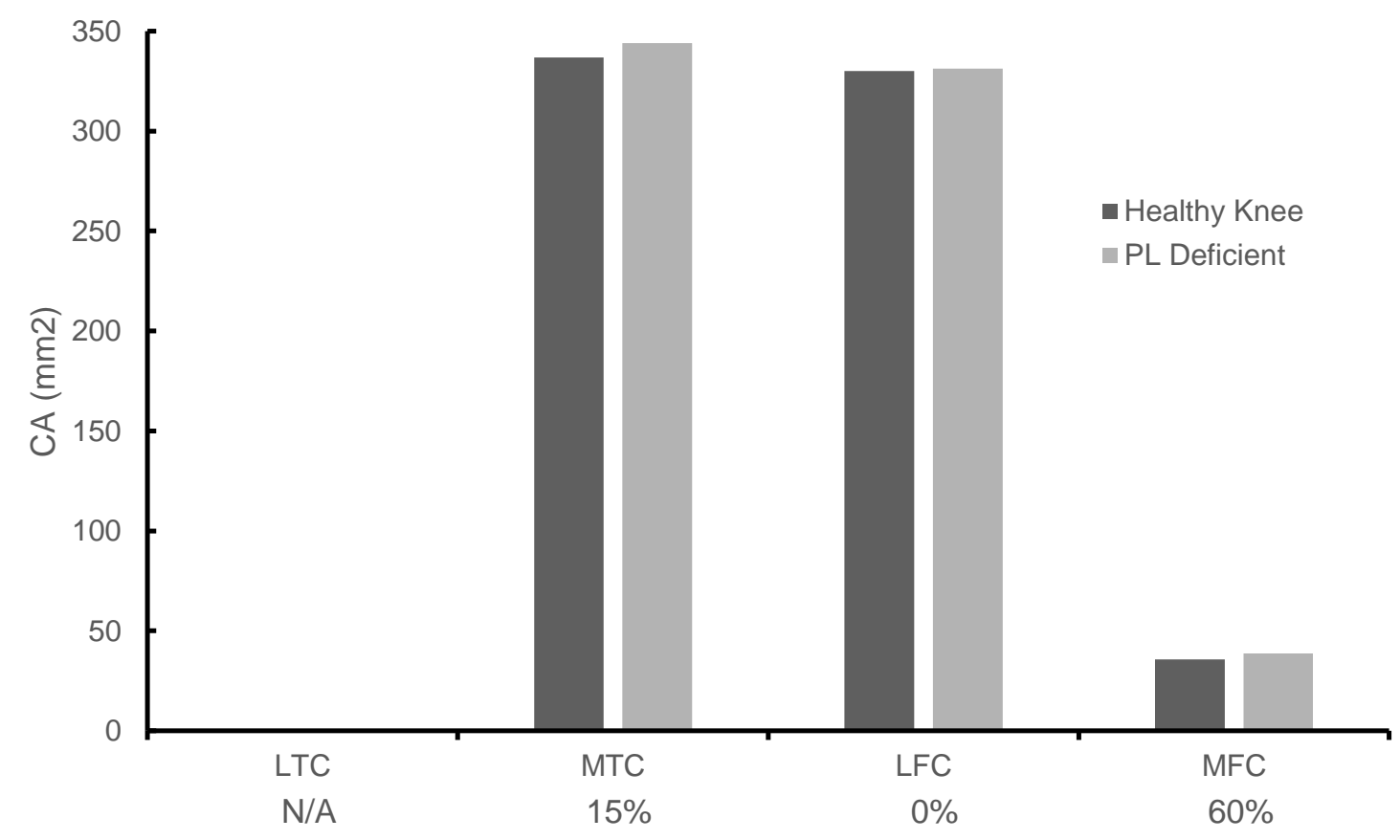

Figure 30: The point of Gait where the largest percent increase of CA occurred for each articular cartilage between intact ACL and PL deficient Knees. The CA of the lateral tibial cartilage decreased at every point of gait for all various types of $A C L$ injuries.

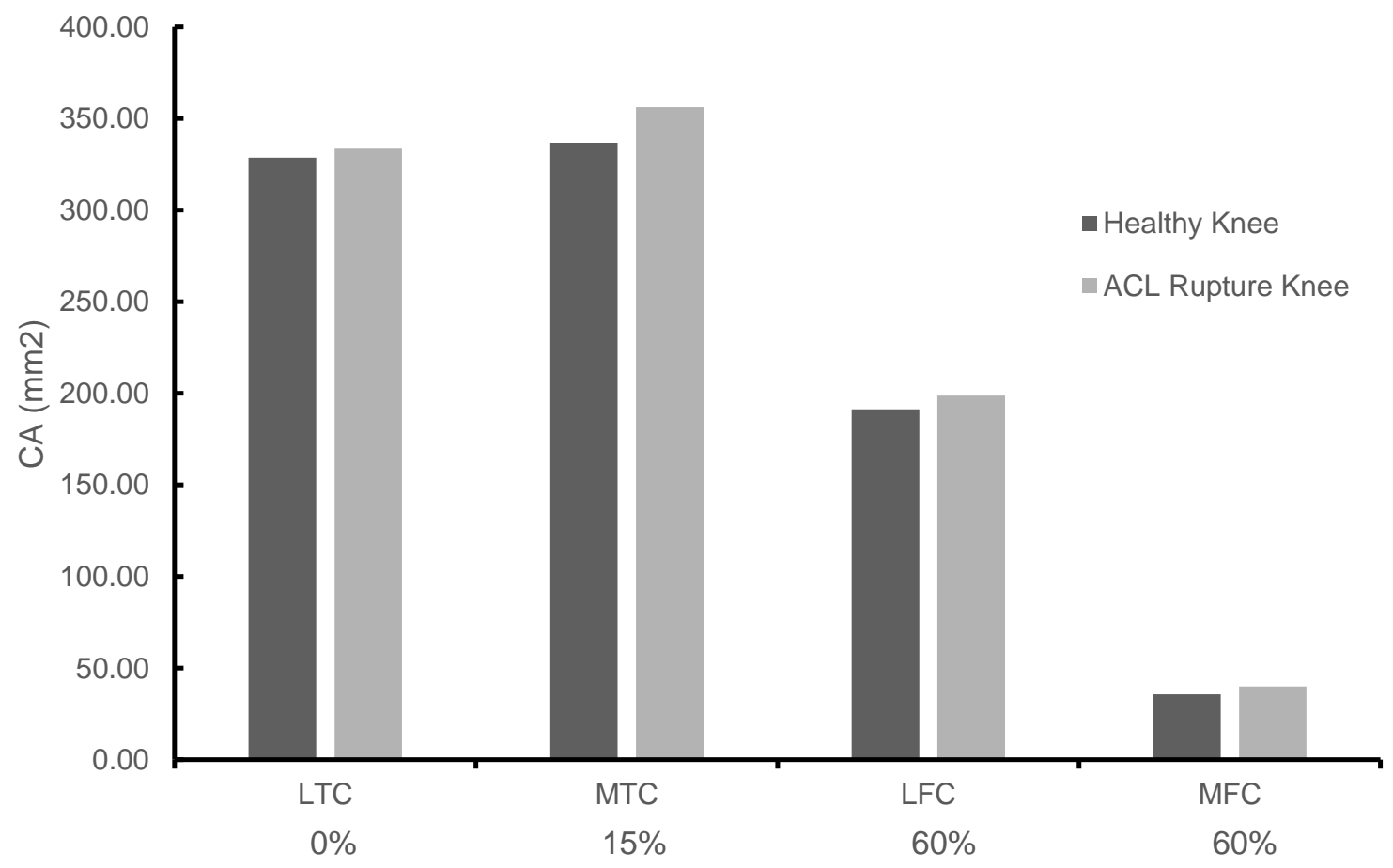

Figure 31: The point of Gait where the largest percent increase of CA occurred for each articular cartilage between intact $A C L$ and $A C L$ ruptured Knees. 


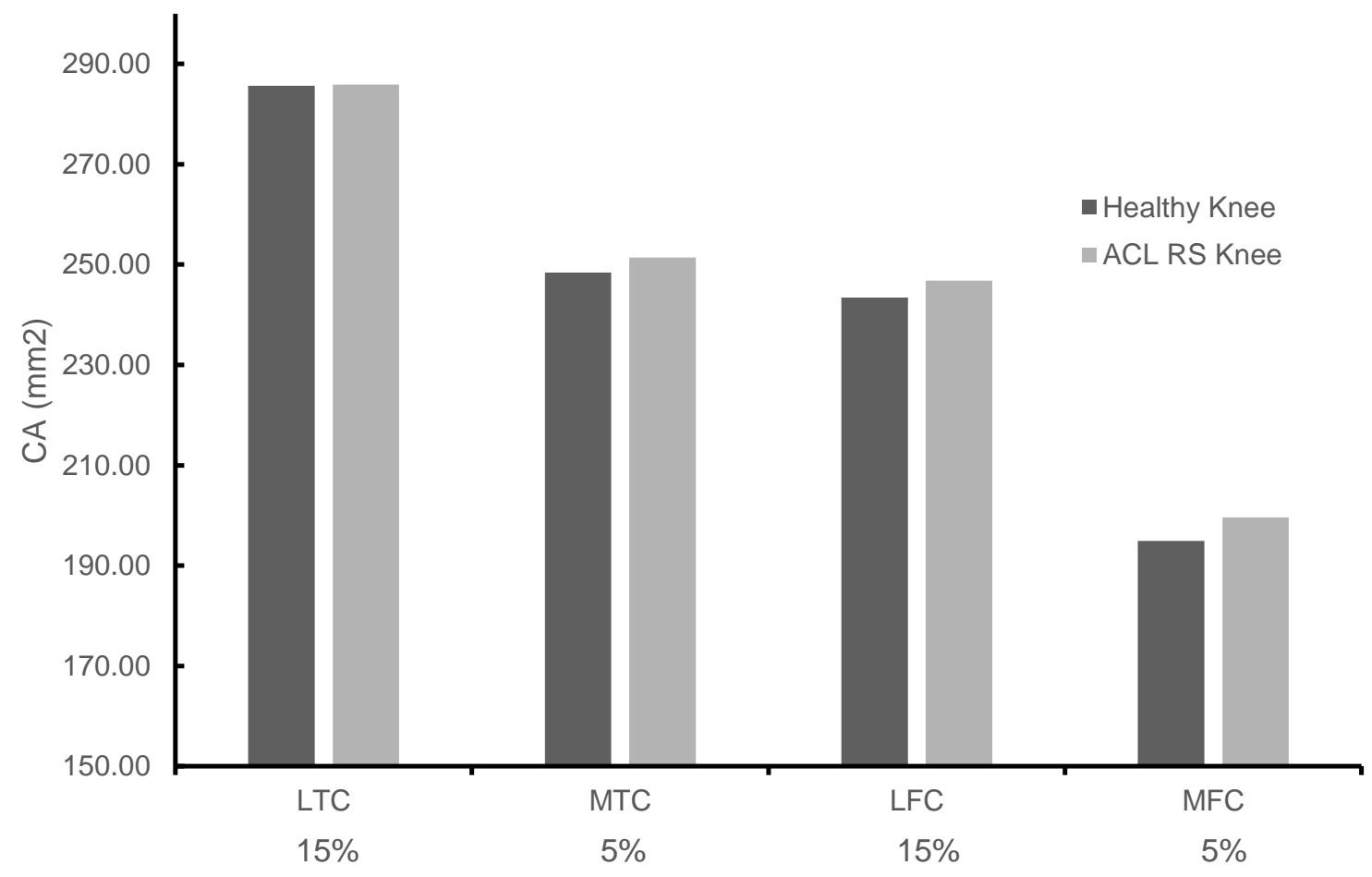

Figure 32: The point of Gait where the largest percent increase of CP occurred for each articular cartilage between intact ACL and ACL RS Knees. 


\section{CHAPTER 4 DISCUSSION}

\subsection{Tibiofemoral Contact Pressure and Contact Area}

The study performed for this thesis was a complex study involving varying loads, boundary conditions and modeled components. While the results reported in Section 3.2 focus on the maximum increase of $\mathrm{CP}$ and $\mathrm{CA}$ for $\mathrm{AM}$ deficient, $\mathrm{PL}$ deficient, $A C L$ ruptured and ACL RS knees for each articular cartilage surface, this may miss other possible conclusions. For this reason the discussion section will focus on the implications of the maximum increase of $\mathrm{CP}$ and $\mathrm{CA}$ for $\mathrm{AM}$ deficient, PL deficient, ACL ruptured and ACL RS knees as well as focus on the change of $\mathrm{CP}$ and CA throughout gait with respect to AM deficient, PL deficient, ACL ruptured and ACL RS knees. Results specifically comparing the changes of CP and CA throughout gait for thesis cases are shown in Appendix C.

4.1.1 Changes to Cartilage Loading with respect to Anterior Cruciate Ligament Deficiency and Reconstruction

Due to the fact that in all four cases the maximum increase of CP for the lateral tibial cartilage is at $46 \%$ of gait and the maximum increase of CP for the lateral portion of the femoral cartilage is at $30 \%$ of gait suggest that the ACL is highly strained throughout this portion of gait. The maximum increase of $\mathrm{CP}$ of ACL RS knees in the lateral compartment was smaller than AM deficient, PL deficient and ACL ruptured maximum increase of $C P$; this suggests that not only is the BPTB graft better for the patient than no ACL, but that the anatomical SB RS mimics behavior of both the AM and PL bundle. 
It is surprising that the maximum increase of CA does not follow the trend of maximum increase of $\mathrm{CP}$ as well, however this suggests that the ACL may be highly strained throughout gait except at certain phases of gait other supporting structures are more readily available. The maximum change of CA for ACL RS is typically smaller than the maximum increase of CA for AM deficient, PL deficient and ACL ruptured knees, only larger than the maximum increase of $C A$ in the lateral tibial cartilage and lateral portion of the femoral cartilage for PL deficient knees. This again suggests that not only is the BPTB better for the patient than no ACL, but that the BPTB graft properly mimics the behavior of the AM and PL bundles for various loading conditions and knee flexion angles. The results that the maximum increase in $\mathrm{CP}$ and $\mathrm{CA}$ occur all throughout gait for the various ACL deficiencies and RS indicate walking with these injuries will increase the risk of developing knee $\mathrm{OA}$, with a complete $\mathrm{ACL}$ ruptured knee the most likely candidate to develop knee OA.

\subsubsection{Changes to Cartilage Loading Throughout Gait}

As mentioned earlier, to view the more detailed results discussed in this section refer to Appendix C.

Loading at $0 \%$ of gait represented heel strike and had a relatively large ratio of anterior tibial force to compressive force. The maximum increase of $\mathrm{CP}$ and $\mathrm{CA}$ tends to change more in PL deficient knees compared to AM deficient knees which agrees with the previously reported notion of the PL bundle supporting more of the load at small knee flexion angles. The CP was higher everywhere and the CA was smaller in all cases except the medial tibial cartilage 
when the BPTB graft was modeled compared to ACL ruptured knees. Generally, the $\mathrm{CP}$ was higher and $\mathrm{CA}$ smaller for all $\mathrm{ACL}$ cases when compared to intact ACLs.

Loading at $5 \%$ of gait was chosen because it represented a relative peak in abduction and tibial internal moments. As with $0 \%$ of gait, AM deficient knees more closely resembled intact $A C L$ knees when compared to $\mathrm{PL}$ deficient knees with respect to $\mathrm{CP}$ and $\mathrm{CA}$. Unlike the predicted results for $0 \%$ of gait, the increase of CP was reduced when the BPTB graft knee was modeled compared to the $\mathrm{ACL}$ ruptured knee. Comparing the predicted results for BPTB graft and PL deficiency shows that the BPTB graft knees had a smaller increase to the CP. This indicates that an anatomic SB BPTB graft can, at least partially, mimic the function of the PL bundle at low knee flexion angles and moderate loading cases.

Loading at $15 \%$ of gait was chosen because of the relative peak in compressive force and adduction moment. This point of gait actually had the least changes between intact and damaged ACLs. This is most likely due to the fact that there is a relative minimum in anterior tibial force and tibial internal moment. None of the CP changed by more than a percent. The CA of the lateral compartment decreased slightly while the medial compartment showed an increase up to $5 \%$ for $\mathrm{ACL}$ ruptured knees. This indicates that there needs to be a combination of loading that activates all three functions of the ACL (restrain ATT, restrict excessive internal-external rotation, and maintain proper varusvalgus alignment) to effectively strain the $A C L$ and produce larger changes in cartilage loading in ACL injured joints. 
Loading at $30 \%$ of gait was chosen because of the relative dip in compressive force and adduction moment, while still maintaining a high tibial internal moment. The increase of CP for tibial cartilage of $\mathrm{AM}$ and $\mathrm{PL}$ deficient is very similar, however there is a much larger increase of $\mathrm{CP}$ for femoral cartilage for PL deficiency compared to AM deficiency. Although the flexion angle is only $12^{\circ}$, the AM bundle appears to be supporting more of the load than would be expected. This could be interpreted as the AM bundle supporting more load at smaller flexion angles with combined loading is concerned. More analyses would have to be done to test this hypothesis. The BPTB graft knee has a markedly smaller increase of the CP compared to the ACL ruptured knee. The predicted increase of CP of a BPTB graft knee is comparable to AM deficient knees for both tibial and femoral articular cartilage. This is an indication that the anatomic SB BPTB graft mimics the behavior of both the AM and PL bundles.

Loading at $46 \%$ of gait was chosen because of the relative peak of compressive force, internal tibial and adduction moments. PL deficient knees showed a larger increase of the maximum tibial and femoral medial portion of the articular cartilage contact pressure. On the contrary, AM deficient knees showed a larger increase of the maximum femoral lateral portion of the articular cartilage contact pressure when compared to $\mathrm{PL}$ deficient knees. This information combined with the fact that the flexion angle at $46 \%$ of gait is only $6^{\circ}$ is another indication that the flexion angle is not the only factor in deciding which bundle supports the majority of the load. The predicted increase of the CP of BPTB graft 
knees was smaller than all other cases except for the medial tibial articular cartilage.

Loading at $60 \%$ of gait represents toe off and has a relatively small compressive force and internal tibial moment. The AM deficient knee had a markedly larger increase of maximum medial tibial articular cartilage contact pressure compared to the $\mathrm{PL}$ deficient knee, however both knees had a decrease of $\mathrm{CP}$ in the lateral compartment. The change of the $\mathrm{CP}$ of the BPTB graft knee followed the same trend as the $\mathrm{AM}$ and $\mathrm{PL}$ deficient knees; not as large of an increase as the AM deficient knee but more than the PL deficient knee. This is interpreted as another indicator that the anatomic SB BPTB graft accurately mimics both the AM and PL bundles.

The reader needs to be reminded that the loading used was from a healthy individual's gait, and as such predicted CP and CA of ACL injuries and reconstruction are not necessarily the actual values experienced by knees with ACL injuries and reconstruction. This is because individuals with $A C L$ injuries or reconstruction have gait adaptations [105], [16], [102] that may change the loading experienced. However, these results are still a good indication of the possible changes to $\mathrm{CP}$ and $\mathrm{CA}$ to those with $\mathrm{ACL}$ injuries and RS. These results indicate that receiving an anatomic SB BPTB graft after a complete ACL rupture helps return the articular cartilage loading back to normal during gait. Multiple points of gait indicated that although this was only a SB RS graft, it mimicked behaviors of both AM and PL bundles. The results also suggest that whichever 
bundle supports the load may be indicated by the type of loading rather than just knee flexion angle.

\subsection{Anterior Tibial Translation}

The predicted results indicate that there is no increase in ATT for any of the ACL deficiencies or BPTB graft. These results are consistent with published reports that show a reduction of ATT throughout all of gait [105]. This is not surprising when the different loads for each point of gait are considered. The $30 \%, 46 \%$ and $60 \%$ points of gait have a posterior tibial force so removing the ACL would not cause any increase of ATT in the model. The $0 \%$ and $5 \%$ points of gait have an anterior tibial force, however there is also an abduction moment that may cause a laxity in the ACL. The $15 \%$ point of gait has a large adduction

moment, however there is a minimal anterior tibial force and internal tibial moment and a relative peak of the compressive force. The combination of these forces may cause a laxity in the ACL, resulting in most of the anterior tibial force being absorbed elsewhere.

\subsection{Viscous Damping Coefficient}

For the majority of simulations the stabilizing viscous forces were less than $1 \%$ of the total forces applied, with the highest percentage of viscous forces reaching $2 \%$ of the total forces applied. These viscous forces to total forces ratio suggest that the specified damping coefficient used did not negatively influence the results. 


\subsection{Future Work}

This FEM and the work developing it signify a considerable step forward toward the long term goal of developing subject specific knee FEMs. The suggestions described below will further increase the accuracy for predicting articular cartilage loading during various exercises.

\subsubsection{Anterior Cruciate Ligament Deficient Gait Loading}

At this point, one of the major limitations to accurately predicting the effect of ACL deficient and ACL RS knees during gait is the lack of usable knee gait loading for individuals suffering from ACL injuries or RS. It is evident that an individual's gait changes after ACL injury and RS [105], [16], [102], however a complete loading cycle of ACL deficient or ACL RS gait was unavailable at this time. Despite this set back, some conclusions can still be drawn from the predicted results. As mentioned earlier, an increase in CP and or CA between injured $A C L$ bundle and intact $A C L$ is considered to be a higher risk of developing $\mathrm{OA}$. However, the larger the increase in $\mathrm{CP}$ or $\mathrm{CA}$, the larger the risk is to develop knee OA. Most of the predicted increase in $\mathrm{CP}, 60 \%$, is less than a $1 \%$ increase, while a staggering $89 \%$ of the predicted increase in CP is less than a $5 \%$ increase. Similar results are seen in the predicted increase of CA, $47 \%$ of the predicted increase is less than a $1 \%$ increase and $93 \%$ of the predicted increase is less than a $5 \%$ increase. Due to the majority of predicted increases being so small, gait is considered to be a safe activity for people with a damaged or reconstructed ACL. Analysis of different activities, such as running, ellipticals, 
cycling and cutting should be performed to determine which activities put the subject most at risk for the development of OA.

\subsubsection{Patellofemoral Joint}

As mentioned earlier, the TF contact loading does not include any forces or moments from the PF joint. A possible solution would be to have OpenSim report the PF contact forces and apply them directly to the femur where appropriate. A better solution would be to include the PF joint in the FEM and apply the quad forces directly to the proximal surface of the patellar tendon. A patellar tendon, patella and patella cartilage were modeled using the same MRIs that generated the rest of the knee FEM, however the PF joint was unable to validate using cadaver studies that measured the CP under various quadriceps loading conditions. Possible remedies for this issue would be to include the medial and lateral patellofemoral ligaments, change the contact scheme between the patella and femoral cartilage, re-evaluate the quality and tibial attachment of the patellar tendon model or change the material definition of the patellar tendon to a hyper-elastic transversely isotropic material.

\subsubsection{Material Properties}

As mentioned earlier, actual articular cartilage, ligaments, menisci and bone are more accurately modeled using more complex material definitions than the ones used in this study. Using a more correct material definition could greatly improve the accuracy of the predicted results. Abaqus has a large material library to choose from and if it does not include the necessary material model then a user-defined material (UMAT) can be built and implemented. 
Articular cartilage is more accurately modeled as a biphasic, poroviscoelastic material [67], whose properties change severely with the alignment of the collagen fibrils and water content in the superficial, transitional and deep zones [106]. Other studies have modeled the articular cartilage using a hyperelastic depth-dependent nonfibrillar matrix and continuum/membrane fibrils [52], [36], [53], a nearly incompressible Mooney-Rivlin [107], and a heterogeneous fibril-reinforced poroviscoelastic [108] material definition.

To more accurately model the ligaments and patellar tendon, previous FE studies have modeled them as hyperelastic, isotropic incompressible NeoHookean [40], as well as hyperelastic, transversely isotropic materials that do not support compressive loads [42], [45]. It is believed that one of the main reasons for convergence difficulties in the FEM was buckling in the ligaments, especially in the $\mathrm{AM}$ and $\mathrm{PL}$ bundles. Preventing the ligaments from supporting compressive loads could minimize, if not completely resolve, the issue of buckling ligaments.

Similar to the articular cartilage, the menisci are much more complicated, showing distinct transversely isotropic, visco- and poroelastic material characteristics [71]. Previous FE studies have modeled the menisci as linearly elastic transversely isotropic [43], [108], transversely isotropic, biphasic [109], and anisotropic with depth-dependent collagen reinforcement [36], [52], [53] materials.

Human bone is actually composed of two sections, a hard outer cortical shell and a softer trabecular center [106]. The outer cortical shell is what was 
simplified to a rigid body due to its transverse isotropy and modulus that increases with loading rate [106]. Previous FE studies of static compressive loading showed that the difference between a rigid body and linear elastic orthotropic model was less than $2 \%$ [43]. However, other FE studies have shown that using this rigid body model in more elaborate loading, such as walking, produced contact pressures and areas much higher than those produced using a more complex model [110], [111].

It should be noted that using these more complex materials could drastically increase the run time of the FEM and possibly prevent it from converging to a solution. A more complex model for the bone will most certainly increase run time because the bones would have to be modeled as 3-D solid elements instead of the shell elements used in this study. Before a more complex material model is adopted, the goal of the study and expected hindrances should be carefully considered.

\subsubsection{Automatic Stabilization Constant Damping Factor}

To ensure that the automatic stabilization is not a dominate factor in the analysis Abaqus recommends checking the ratio of viscous forces to total forces applied. However, it also recommends checking the ratio of the energy dissipated due to the automatic stabilization to the internal strain energy. If the ratio of energies is below the Abaqus recommended .0002, then the automatic stabilization likely didn't dominate the analysis. When the ratio was checked for all the studies it varied from .75 to 32 . 
An energy ratio this large suggests that this TF joint isn't modeled properly. However, this was known before any analysis was performed. The loads applied to this FEM represent the loads of a healthy patient throughout gait. In reality, this patient has a PF joint, the meniscal horns have physical size, the MCL and PCL are actually composed of two bundles, there is friction between all contacting structures, ligaments are surrounded in synovial fluid, and there are entire ligaments that are missing from the model. Due to this simplified model of the human knee joint, an automatic stabilization factor with a larger than preferred energy ratio is considered an acceptable method to produce the predicted results. 


\section{CHAPTER 5 CONCLUSION}

The specific goals of this thesis were to 1) develop and validate a complete FEM of the tibiofemoral joint using a two-bundle model of the ACL and 2) predict the change of articular cartilage tissue loading of the knee with a healthy, ACL injured and BPTB graft using the loading conditions of a healthy person's gait. Using multiple loading conditions and boundary conditions, the FEM was considered successfully validated for CP and ATT. The predicted results of the gait analysis indicate that while CP increases and CA decreases when modeled with an anatomic SB BPTB graft, the changes are not as drastic as changes seen when a complete $A C L$ rupture is modeled. The results also indicate that while the anatomic SB BPTB may not completely replace both AM and PL bundles, it supports loads typically supported by only the AM or PL bundle in an intact ACL. The results also suggest that whichever bundle supports the majority of the load may be more influenced by combination loading and knee flexion angle rather than just knee flexion angle. While CP and CA increased for most of gait for all ACL injuries, the amount of increase suggests that walking is still a safe activity for individuals with injured ACLs.

The long term goal of this project is to develop, validate and use subjectspecific FEM of the human knee in clinical applications to determine patient specific short-term rehabilitative and long-term fitness sustainment exercises that

may prevent the onset, or progression, of $\mathrm{OA}$ in patients with $\mathrm{ACL}$ injured or reconstructed knees. With the tools learned in the development of the FEM, 
along with the suggested improvements, this goal is manageable with continual work. 


\section{BIBLIOGRAPHY}

[1] "Osteoarthritis," Centers for Disease Control and Prevention, 16 May 2014. [Online].

Available:

http://www.cdc.gov/arthritis/basics/osteoarthritis.htm. [Accessed 2014 August 2014].

[2] "Data and Statistics," Centers for Disease and Control and Prevention, 10 February $2014 . \quad$ [Online]. Available: http://www.cdc.gov/arthritis/data_statistics.htm. [Accessed 8 August 2014].

[3] H. Kotlarz, C. L. Gunnarsson and et al., "Insurer and Out-of-Pocket Costs of Osteoarthritis in the US," Arthritis and Rheumatology, vol. 60, no. 12, pp. 3546-3553, 2009.

[4] "Arthritis Related Stats," Centers for Disease Control and Prevention, 17 March $2014 . \quad$ [Online]. Available: http://www.cdc.gov/arthritis/data_statistics/arthritis_related_stats.htm \#12. [Accessed 8 August 2014].

[5] "What is Osteoarthritis," Arthritis Foundation, 10 February 2012. [Online]. Available: http://www.arthritistoday.org/about-arthritis/types-ofarthritis/osteoarthritis/what-you-need-to-know/osteoarthritis-is.php. [Accessed 11 August 2014].

[6] D. T. Felson, Y. Zhang, M. T. Hannan and et al., "The incidence and natural history of knee osteoarthritis in the elderly. The Framingham Osteoarthritis Study.," Arthritis and Rheumatology, vol. 38, pp. 15001505, 1995.

[7] N. Sabeka and L. Sharma, "Epidemiology of Osteoarthritis: An Update," Current Rheumatology Reports, vol. 8, pp. 7-15, 2006.

[8] M. Dougados, "Why and How to Use NSAIDs in Osteoarthritis," J Cardiovasc Pharmacol, vol. 47, no. 1, pp. 49-54, 2006.

[9] "Arthritis of the Knee -- Total Knee Replacements (Arthroplasty) and Other Treatment at HSS.," Hospital for Special Surgery, 14 June 2010. [Online]. Available: http://www.hss.edu/conditions_arthritis-of-theknee-total-knee-replacement.asp\#.U-U7OONdWSo. [Accessed 8 August 2014]. 
[10] B. B. P, G. D. S, J. F. A and et al., "Mechanisms of Anterior Cruciate Ligament Injury," Orthopedics, vol. 23, no. 6, pp. 573-578, 2000.

[11] A. M. Chaudhari, P. L. Briant, S. L. Bevill and et al., "Knee kinematics, cartilage morphology, and osteoarthritis after ACL injury," Medicine and Science in Sports and Exercise, vol. 40, no. 2, pp. 215-222, 2008.

[12] D. T. Felson, "Epidemiology of hip and knee Osteoarthritis," Epidemiologic Reviews, vol. 10, pp. 1-28, 1988.

[13] D. T. Felson, Y. Zhang, J. M. Anthony and et al., "Weight Loss Reduces the Risk for Symptomatic Knee Osteoarthritis in Women: The Framingham Study," American College of Physicians, vol. 116, no. 7, pp. 535-539, 1992.

[14] J. A. Buckwalter and H. J. Mankin, "Articular cartilage: degeneration and osteoarthritis, repair, regeneration and transplantation," Instructional Course Lectures, vol. 47, pp. 487-504, 1998.

[15] N. Sabeka and L. Sharma, "Epidemiology of Osteoarthritis: An Update," Current Rheumatology Reports, vol. 8, pp. 7-15, 2006.

[16] D. M. Daniel, M. L. Stone, B. E. Dobson and et al., "Fate of the ACLinjuries patient: A prospective outcome study," American Journal of Sports Medicine, vol. 22, pp. 632-644, 1994.

[17] "Anterior Cruciate Ligament Injury," Department of Orthopedic Surgery, UCSF, January $2009 . \quad$ [Online]. Available: http://orthosurg.ucsf.edu/patient-care/divisions/sportsmedicine/conditions/knee/anterior-cruciate-ligament-injury-acl. [Accessed 20 August 2014].

[18] "Anterior Cruciate Ligament (ACL) Injuries," The American Orthopaedic Society for Sports Medicine, March 2014. [Online]. Available: http://orthoinfo.aaos.org/topic.cfm?topic=a00549. [Accessed 11 August 2014].

[19] T. M. Davidson and A. T. Laliotis, "Alpine skiing injuries. A nine-year study.," The Western Journal of Medicine, vol. 164, no. 4, pp. 310314, 1996.

[20] J. Grant and R. L. Kirby, "Anterior Cruciate: Methods of Physical Examination," The College of Family Physicians of Canada, vol. 28, pp. 311-314, 1982. 
[21] M. K. Kwan, T. H. Lin and S. L. Woo, "On the Viscoelastic properties of the Anteromedial Bundle of the Anterior Cruciate Ligament," Journal of Biomechanics, vol. 26, no. 4/5, pp. 447-452, 1993.

[22] S. Kopf, V. Musahl, S. Tashman and et al., "A Systematic Review of the Femoral Origin and Tibial Insertion Morphology of the ACL," Knee Surg Traumatol Arthrosc, vol. 17, pp. 213-219, 2009.

[23] M. Sakane, R. J. Fox, L. Y. Savio and et al., "In situ forces in the anterior cruciate ligament and its bundles in response to anterior tibial loads.," Journal of Orthopaedic Research, vol. 15, no. 2, pp. 285293, 1997.

[24] W. J. Sebastianelli, "American Orthopaedic Society for Sports Medicine," 2008. [Online].

Available: https://www.sportsmed.org/uploadedFiles/Content/Patient/Sports_Ti ps/ST\%20Injured\%20ACL\%2008.pdf. [Accessed 7 April 2014].

[25] C. D. Harner and G. G. Poehling, "Double Bundle or Double Trouble?," Journal of Arthroscopic and Related Surgery, vol. 20, no. 10, pp. 1013-1014, 2004.

[26] K. Hara, T. Kuno, T. Suginoshita and et al., "Reconstruction of the anterior cruciate ligament using a double bundle," The Journal of Arthroscopic and Related Surgery, vol. 16, no. 8, pp. 860-864, 2000.

[27] T. Zantop, S. Kuno, W. Petersen and et al., "Current Techniques in Anatomic Anterior Cruciate Ligament Reconstructuion," The Journal of Arthroscopic and Related Surgery, vol. 23, no. 9, pp. 938-947, 2007.

[28] V. Musahl, A. Plakseychuk, A. VanScyoc and et al., "Varying Femoral Tunnels Between the Anatomical Footprint and Isometric Positions: Effect on Kinematics of the Anterior Cruciate LigamentReconstructed Knee," American Journal of Sports Medicine, vol. 33, no. 5, pp. 712-718, 2005.

[29] D. W. Jackson, E. S. Grood, J. D. Goldstein and et al., "A comparison of patellar tendon autograft and allograft used for anterior cruciate ligament reconstruction in the goat model," American Journal of Sports Medicine, vol. 21, no. 2, pp. 176-185, 1993.

[30] R. Siebold, J. U. Buelow, L. Bos and et al., "Primary ACL reconstruction with fresh-frozen patellar versus Achilles tendon allografts," Archives of Orthopaedic and Trauma Surgery, vol. 123, no. 4, pp. 180-185, 2003. 
[31] M. J. Goertzen, H. Clahsen, K. F. Burrig and et al., "Anterior cruciate ligament reconstruction using cryopreserved irradiated bone-ACLbone-allograft transplants," Knee Surgery Sports Traumatology Arthroscopy, vol. 2, no. 3, pp. 150-157, 1994.

[32] W. R. Taylor, M. O. Heller, G. Bergmann and et al., "Tibio-femoral loading during human gait and stair climbing," Journal of Orthopaedic Research, vol. 22, pp. 625-632, 2004.

[33] C. R. Winby, D. G. Lloyd, T. F. Besier and et al., "Muscle and external load contribution to knee joint contact loads during normal gait," Journal of Biomechanics, vol. 42, no. 14, pp. 2294-2300, 2009.

[34] M. Z. Bendjaballah, A. Shirazi-Adl and D. J. Zukor, "Biomechanics of the human knee joint in compression: reconstruction, mesh generation and finite element analysis," The Knee, vol. 2, no. 2, pp. 69-79, 1995.

[35] M. Adouni and A. Shirazi-Adl, "Evaluation of Knee Joint Forces and Tissue Stresses-Strains during Gait in Severe OA versus Normal Subjects," Journal of Orthopaedic Research, vol. 32, pp. 69-78, 2014.

[36] R. Shirazi, A. Shirazi-Adl and M. Hurtig, "Role of cartilage collagen fibrils networks in knee joint biomechanics under compression," Journal of Biomechanics, vol. 41, pp. 3340-3348, 2008.

[37] M. Adouni, A. Shirazi-Adl and R. Shirazi, "Computational biodynamics of human knee joint in gait: From muscle forces to cartilage stresses.," Journal of Biomechanics, vol. 45, pp. 2149-2156, 2012.

[38] S. Arno, P. S. Walker, C. P. Bell and et al., "Relationship between cartilage volume and meniscal contact in medial osteoarthritis of the knee.," The Knee, vol. 19, pp. 896-901, 2012.

[39] D. Périé and M. C. Hobatho, "In vivo determination of contact areas and pressure of the femoraotibial joint using non-linear finite element analysis," Clinical Biomechanics, vol. 13, pp. 394-402, 1998.

[40] E. Peña, B. Calvo, M. A. Martinez and et al., "Finite element analysis of the effect of meniscal tears and meniscectomies on human knee biomechanics," Clinical Biomechanics, vol. 13, pp. 498-507, 2005.

[41] Y. Guo, X. Zhang and W. Chen, "Three-Dimensional Finite Element Simulation of Total Knee Joint in Gait Cycle.," Acta Mechanica Solida Sinica, vol. 22, no. 4, pp. 347-351, 2009. 
[42] E. Peña, B. Calvo, M. A. Martinez and et al., "A three-dimensional finite element analysis of the combined behavior of ligaments and menisci in the healthy human knee joint.," Journal of Biomechanics, vol. 39, pp. 1686-1701, 2006.

[43] T. L. Haut Donahue, M. L. Hull, M. M. Rashid and et al., "A Finite Element Model of the Human Knee Joint for the Study of Tibio-Femoral Contact.," Journal of Biomechanical Engineering, vol. 124, pp. 273280, 2002.

[44] Y. Song, R. E. Debski and V. Musahl, "A three-dimensional finite element model of the human anterior cruciate ligament: a computational analysis with experimental validation.," Journal of Biomechanics, vol. 37, no. 3, pp. 383-390, 2004.

[45] E. Peña, M. A. Martinez, B. Calvo and et al., "A finite element simulation of the effect of graft stiffness and graft tensioning in ACL reconstruction.," Clinical Biomechanics, vol. 20, no. 6, pp. 636-644, 2005.

[46] H. Y. Kim, Y. J. Seo, H. J. Kim and et al., "Tension Changes within the Bundles of Anatomic Double-Bundle Anterior Cruciate Ligament Reconstruction at Different Knee Flexion Angles: A Study Using a 3Dimensional Finite Element Model.," Journal of Arthroscopic and Related Surgery, vol. 27, no. 10, pp. 1400-1408, 2011.

[47] J. Suggs, C. Wang and G. Li, "The effect of graft stiffness on knee joint biomechanics after ACL reconstruction -- a 3D computational simulation.," Clinical Biomechanics, vol. 18, no. 1, pp. 35-43, 2003.

[48] M. Kazemi, L. P. Li, P. Savard and et al., "Creep behavior of the intact and meniscectomy knee joints," Journal of the Mechanical Behavior of Biomedical Materials, vol. 4, no. 7, pp. 1351-1358, 2011.

[49] G. Li, O. Lopez and H. Rubash, "Variability of a Three-Dimensional Finite Element Model Constructed Using Magnetic Resonance Images of a Knee for Joint Contact Stress Analysis," Journal of Biomechanical Engineering, vol. 123, pp. 341-346, 2001.

[50] N. A. Ramaniraka, P. Saunier, O. Siegrist and et al., "Biomechanical evaluation of intra-articular and extra-articular procedures in anterior cruciate ligament reconstruction: A finite element analysis," Clinical Biomechanics, vol. 22, no. 3, pp. 336-343, 2007.

[51] M. Z. Bendjaballah, A. Shirazi-Adl and D. J. Zukor, "Finite element analysis of human knee joint in varus-valgus," Clinical Biomechanics, vol. 12, 
no. 3, pp. 139-148, 1997.

[52] R. Shirazi and A. Shirazi-Adl, "Computational biomechanics of articular cartilage of human knee joint: Effect of osteochodral defects," Journal of Biomechanics, vol. 42, no. 15, pp. 2458-2465, 2009.

[53] R. Shirazi and A. Shirazi-Adl, "Analysis of partial meniscectomy and ACL reconstruction in knee joint biomechanics under combined loading.," Clinical Biomechanics, vol. 24, no. 9, pp. 755-761, 2009.

[54] R. Siebold, T. Ellert, S. Metz and e. al., "Tibial inerstions of the anteromedial and posterolateral bundles of the anterior cruciate ligament: morphometry, arthroscopic landmarks, and orientation model for bone tunnel placement," Arthroscopy, vol. 24, no. 2, pp. 154-161, 2008.

[55] M. Takahashi, M. Doi, M. Abe and et al., "Anatomical study of the femoral and tibial insertions of the anteromedial and posterolateral bundles of human anterior cruciate ligament.," American Journal of Sports Medicine, vol. 34, no. 5, pp. 787-792, 2006.

[56] R. Siebold, T. Ellert, S. Metz and e. al., "Femoral insertions of the anteromedial and posterolateral bundles of the anterior cruciate ligament: morphometry and arthroscopic orientation models for double-bundle bone tunnel placement--a cadaver study," Arthroscopy, vol. 24, no. 5, pp. 585-592, 2008.

[57] N. A. Mall, A. S. Lee, B. J. Cole and et al., "The Functional and Surgical Anatomy of the Anterior Cruciate Ligament," Operative Techniques in Sports Medicine, vol. 21, no. 1, pp. 2-9, 2013.

[58] G. Y. Ng, B. W. Oakes, O. W. Deacon and et al., "Biomechanics of Patellar Tendon Autograft for Reconstruction of the ANterior Cruciate Ligament in the Goat: Three-Year Study.," Journal of Orthopaedic Research, vol. 13, pp. 602-608, 1995.

[59] R. T. Ballock, S. L.-Y. Woo, R. M. Lyon and et al., "Use of Patellar Tendon Autograft for Anterior Cruciate Ligament Reconstruction in the Rabbit: A Long-Term Histologic and Biomechanical Study.," Journal of Orthopaedic Research, vol. 7, pp. 474-485, 1989.

[60] R. F. LaPrade, T. V. Ly, F. A. Wentorf and et al., "The posterolateral attachments of the knee: a qualitative and quantitative morphologic analysis of the fibular colalteral ligament, popliteus tendon, popliteofibular ligament, and lateral gastrocnemius tendon.," American Journal of Sports Medicine, vol. 31, no. 6, pp. 854-860, 
2003.

[61] B. R. Meister, S. P. Michael, R. A. Moyer and et al., "Anatomy and kinematics of the lateral collateral ligament of the knee.," American Journal of Sports Medicine, vol. 28, no. 6, pp. 869-878, 2000.

[62] J. M. Brinkman, P. J. Schwering, L. Blankevoort and et al., "The insertion geometry of the posterolateral corner of the knee," Journal of Bone and Joint Surgery, vol. 87, pp. 1364-1368, 2005.

[63] R. F. LaPrade, A. H. Engebretsen, T. V. Ly and et al., "The Anatomy of the Medial Part of the Knee," The Journal of Bone and Joint Surgery, vol. 89, no. 9, pp. 2000-2010, 2007.

[64] F. Liu, B. Yue, H. R. Gadikota and et al., "Morphology of the medial collateral ligament of the knee," Journal of Orthopedic Surgery and Research, vol. 5, no. 69, pp. 1-8, 2010.

[65] J. R. Robinson, A. M. Bull and A. A. Amis, "Structural properties of the medial collateral ligament complex of the human knee," Journal of Biomechanics, vol. 38, no. 5, pp. 1067-1074, 2005.

[66] N. Yang, P. Canavan, H. Nayeb-Hashemi and et al., "Protocal for constructing subject=specific biomechanical models of knee joint," Computer Methods in Biomechanics and Biomedical Engineering, vol. 13, no. 5, pp. 589-603, 2010.

[67] M. R. DiSilvestro, Q. Zhu, M. Wong and et al., "Biphasic Poroviscoelastic Simulation of the Unconfined Compression of Articular Cartilage: 1-Simultaneous Prediction of Reaction Force and Lateral Displacement," Journal of Biomechanical Engineering, vol. 123, pp. 191-197, 2001.

[68] S. S. Chen, Y. H. Falcovitz, R. Schneidermant and et al., "Depthdependent compressive properties of normal aged human femoral head articular cartilage: relationship to fixed charge density," Journal of the OsteoArthritis Research Society International, vol. 9, pp. 561569, 2001.

[69] C. Y. Huang, V. C. Mow and G. A. Ateshian, "The Role of FlowIndependent Viscoelasticity in the Biphasic Tensile and Compressive Responses of Articular Cartilage.," Journal of Biomechanical Engineering, vol. 123, pp. 410-417, 2001.

[70] C. G. Armstrong, W. M. Lai and V. C. Mow, "An Analysis of the Unconfined Compression of Articular Cartilage," Journal of Biomechanical 
Engineering, vol. 106, no. 2, pp. 165-173, 1984.

[71] E. K. Danso, J. T. A. Mäkelä, P. Tanska and et al., "Characterization of site-specific biomechanical properties of human meniscus-Importance of collagen and fluid on mechanical nonlinearities.," Journal of Biomechanics, pp. 1-9, 2015.

[72] D. L. Butler, M. D. Kay and D. C. Stouffer, "Comparison of material properties in fascicle-bone units from human patellar tendon and knee ligaments," Journal of Biomechanics, vol. 19, no. 6, pp. 425432, 1986.

[73] K. M. Quapp and J. A. Weiss, "Material charaterization of human medial collateral ligament.," Journal of Biomechanical Engineering, vol. 120, no. 6, pp. 757-763, 1990.

[74] D. L. Butler, Y. Guan, M. D. Kay. and et al., "Location-dependent variations in the material properties of the anterior cruciate ligament," Journal of Biomechanics, vol. 25, no. 5, pp. 511-518, 1992.

[75] F. R. Noyes and E. S. Grood, "The strength of the anterior cruciate ligament in humans and Rhesus monkeys.," Journal of Bone and Joint Surgery, vol. 58, no. 8, pp. 1074-1082, 1976.

[76] N. Chandrashekar, H. Mansouri, J. Slauterbeck and et al., "Sex-based differences in the tensile proerties of the human anterior cruciate ligament.," Journal of Biomechanics, vol. 39, no. 16, pp. 2943-2950, 2006.

[77] J. Hewitt, F. Guilak, R. Glisson and et al., "Regional material properties of the human hip joint capsule ligaments.," Journal of Orthopaedic Research, vol. 19, pp. 359-364, 2001.

[78] J. A. Weiss, J. C. Gardiner and C. Bonifasi-Lista, "Ligament material behavior is nonlinear, viscoelastic and rate-independent under shear loading.," Journal of Biomechanics, vol. 35, no. 7, pp. 943-950, 2002.

[79] J. Hashemi, N. Chandrashekar and J. Slauterbeck, "The mechanical properties of the human patellar tendon are correlated to its mass density and are independent of sex," Clinical Biomechanics, vol. 20, no. 6, pp. 645-652, 2005.

[80] G. A. Johnson, D. M. Tramaglini, R. E. Levine and et al., "Tensile and Viscoelastic Properties of Human Patellar Tendon," Journal of Orthopaedic Research, vol. 12, no. 6, pp. 796-803, 1994. 
[81] D. L. Butler, E. S. Grood, F. R. Noyes and et al., "Mechanical Properties of Primate Vascularized vs. Nonvascularized Patellar Tendon Grafts; Changes Over Time," Journal of Orthopaedic Research, vol. 7, no. 1, pp. 68-79, 1989.

[82] S. D. Abramowitch, C. D. Papageorgiou, J. D. Withrow and et al., "The effect of initial graft tension on the biomechanical properties of a healing ACL replacement graft: a study in goats.," Journal of Orthopaedic Research, vol. 21, pp. 708-715, 2003.

[83] R. T. Ballock, S. L.-Y. Woo, R. M. Lyon and et al., "Use of Patellar Tendon Autograft for Anterior Cruciate Ligament Reconstruction in the Rabbit: A Long-Term Histologic and Biomechanical Study.," Journal of Orthopaedi Research, vol. 7, no. 4, pp. 474-485, 1989.

[84] T. A. L. Wren, S. A. Yerby, G. S. Beaupré and et al., "Mechanical properties of the human achilles tendon.," Clinical Biomechanics, vol. 16, no. 3, pp. 245-251, 2001.

[85] A. Weiler, G. Peters, J. Mäurer and et al., "Biomechanical Properties and Vascularity of an Anterior Cruciate Ligament Graft Can Be Predicted by Contrast-Enhanced Magnetic Resonance Imaging.," American Journal of Sports Medicine, vol. 29, no. 6, pp. 751-761, 2001.

[86] T. Haut-Donahue, M. Hull, M. Rashid and et al., "How the stiffness of meniscal attachments and meniscal material properties affect tibiofemoral contact pressure computed using a validated finite element model of the human knee joint.," Journal of Biomechanics, vol. 36, no. 1, pp. 19-34, 2003.

[87] K. N. Hauch, D. F. Villegas and T. L. Haut-Donahue, "Geometry, timedependent and failure properties of human meniscal attachments.," Journal of Biomechanics, vol. 43, no. 3, pp. 463-468, 2010.

[88] S. Delp, F. Anderson, A. Arnold and et al., "OpenSim: open-source software to create and analyze dynamic simulations of movement.," IEEE Transactions on Biomechanical Engineering, vol. 54, no. 11, pp. 1940-1950, 2007.

[89] S. Wangerin, "Development and Validation of a Human Knee Joint Finite Element Model for Tissue Stress and Strain Predictions During Exercise," California Polytechnic State University, San Luis Obispo, 2013.

[90] J. P. Holden and S. J. Stanhope, "The effect of variation in knee center location estimates on net knee joint moments.," Gait and Posture, 
vol. 7, no. 1, pp. 1-6, 1998.

[91] S. Koo and T. P. Andriacchi, "The knee joint center of rotation is predominantly on th elateral side during normal walking.," Journal of Biomechanics, vol. 41, no. 6, pp. 1269-1273, 2008.

[92] A. M. Seitz, A. Lubomierski, B. Friemert and et al., "Effect of Partial Meniscectomy at the Medial Posterior Horn on Tibiofemoral Contact Mechanics and Meniscal Hoop Strains in Human Knees.," Journal of Orthopaedic Research, vol. 30, no. 6, pp. 934-942, 2012.

[93] Y. Morimoto, M. Ferretti, M. Ekdahl and et al., "Tibiofemoral Joint Contact Area and Pressure After Single- and Double-Bundle Anterior Cruciate Ligament Reconstruction.," Journal of Arthroscopic and Related Surgery, vol. 25, no. 1, pp. 62-69, 2009.

[94] G. S. Berns, M. L. Hull and H. A. Patterson, "Strain in the anteromedial bundle of the anterior curciate ligament under combination loading.," Journal of Orthopaedic Research, vol. 10, no. 2, pp. 167-176, 1992.

[95] S. Lenschow, T. Zantop, A. Weimann and et al., "Joint kinematics and in situ forces after single bundle PCL reconstruction: a graft placed at the center of the femoral attachment does not restore normal posterior laxity.," Archives of Orthopaedic and Trauma Surgery, vol. 126, no. 4, pp. 253-259, 2006.

[96] W. Petersen, S. Lenschow, A. Weimann and et al., "Importance of femoral tunnel placement in double bundle PCL reconstruction: biomechanical analysis using a robotic/universal force moment sensor.," American Journal of Sports Medicine, vol. 34, no. 3, pp. 456-463, 2006.

[97] M. Berchuck, T. P. Andriacchi, B. R. Bach and et al., "Gait adaptations by patients who have a deficient anterior cruciate ligament.," Journal of Bone and Joint Surgery, vol. 72, no. 6, pp. 871-877, 1990.

[98] P. Bulgheroni, M. V. Bulgheroni, L. Andrini and et al., "Gait patterns after anterior cruciate ligament reconstruction," Knee Surgery Sports Traumatology Arthroscopy, vol. 5, no. 1, pp. 14-21, 1997.

[99] P. Devita, T. Hortobagyi and J. Barrier, "Gait biomechanics are not normal after anterior cruciate ligament reconstruction and accelerated rehabilitation.," Journal of the American College of Sports Medicine, pp. 1481-1488, 1998.

[100] F. R. Noyes, O. D. Schipplein, T. P. Andriacchi and et al., "The anterior 
cruciate ligament-deficient knee with varus alignment: An analysis of gait adaptations and dynamic joint loadings.," American Journal of Sports Medicine, vol. 20, no. 6, pp. 707-716, 1992.

[101] R. Shiavi, T. Limbird, M. Frazer and et al., "Helical motion analysis of the knee--2. Kinematics of uninjured and injured knees during walking and pivoting.," Journal of Biomechanics, vol. 20, no. 7, pp. 653-665, 1987.

[102] G. Wexler, D. E. Hurwitz, C. A. Bush-Joseph and et al., "Functional Gait Adaptations in Patients With Anterior Cruciate Ligament Deficiency Over Time.," Clinical Orthopaedics and Related Research, vol. 348, pp. 166-175, 1998.

[103] D. J. Haight, Z. F. Lerner, W. J. Board and et al., "A comparison of slow, uphill and fast, level walking on lower extremity biomechanics and tibiofemoral joint loading in obese and nonobese adults.," Journal of Orthopaedic Research, vol. 32, no. 2, pp. 324-330, 2014.

[104] Y.-C. Lin, J. P. Walter, S. A. Banks and et al., "Simultaneous prediction of muscle and contact forces in the knee during gait.," Journal of Biomechanics, vol. 43, pp. 945-952, 2010.

[105] B. Gao and N. Zheng, "Alterations in three-dimensional joint kinematics of anterior cruciate ligament-deficient and -reconstructed knees during walking.," Clinical Biomechanics, vol. 25, pp. 222-229, 2010.

[106] D. Bartel, D. Davy and T. Keaveny, Orthopaedic Biomechanics: Mechanics and Design in Musculoskeletal Systems, Upper Saddle River, NJ: Pearson Education, Inc., 2006.

[107] S. Sibole, C. Bennett, S. Maas and et al., "Open Knee: a 3D finite element representation of the knee joint," in 34th Annual Meeting of the American Society of Biomechanics, 2010.

[108] M. Mononen, M. Mikkola, P. Julkunen and et al., "Effect of superficial collagen patterns and fibrillation of femoral articular cartilage on knee joint mechanics - a 3D finite element analysis," Journal of Biomechanics, vol. 45, no. 3, pp. 579-587, 2011.

[109] R. L. Spilker, P. R. Donzelli and V. C. Mow, "A Transversely Isotropic Biphasic Finite Element Model of the Meniscus," Journal of Biomechanics, vol. 25, no. 9, pp. 1027-1045, 1992.

[110] A. E. Anderson, B. J. Ellis, S. A. Maas and et al., "Validation of finite element predictions of cartilage contact pressure in the human hip 
joint," Journal of Biomechanical Engineering, vol. 130, no. 5, pp. 051008-1 - 051008-10, 2008.

[111] A. Anderson, B. Ellis, S. Maas and et al., "Effects of idealized joint geometry on finite element predictions of cartilage contact stresses in the hip," Journal of Biomechanics, vol. 43, no. 7, pp. 1351-1357, 2010.

[112] "Funded Injury Control Research Centers (ICRCs)," Centers for Disease Control and Prevention, 13 July 2010. [Online]. Available: http://www.cdc.gov/injury/erpo/icrc/2009/1-R49-CE001495-01.html. [Accessed 20 August 2014].

[113] M. R. DiSilvestro and J. F. Suh, "Biphasic Poroviscoelastic Simulation of the Unconfined Compression of Articular Cartilage: 2--Effect of Variable Strain Rates.," Journal of Biomechanical Engineering, vol. 123, pp. 198-200, 2001.

[114] M. S. DeMers, S. Pal and S. L. Delp, "Changers in Tibiofemoral Forces due to Variations in Muscle Activity during Walking.," Journal of Orthopaedic Research, pp. 1-8, 2014.

[115] M. P. Arnold, N. Verdonschot and A. van Kampen, "ACL graft can replicate the normal ligament's tension curve," Knee Surgery, Sports Traumatology, Arthroscopy, vol. 13, pp. 625-631, 2005.

[116] H. S. Han, S. C. Seong, S. Lee and et al., "Anterior Cruciate Ligament Reconstructuion," Clinical Orthopaedics and Related Research, vol. 466, pp. 198-204, 2008. 


\section{APPENDICES}

\section{APPENDIX A: AUTOMATIC STABILIZATION}

Automatic stabilization by defining a constant damping factor works by including a fictitious viscous force, $F_{V}$, to the global equilibrium equations.

$$
\begin{gathered}
F_{v}=c M^{*} v \\
P-I-F_{v}=0
\end{gathered}
$$

Where $M^{*}$ is an artificial mass matrix calculated with unity density, $c$ is the damping factor specified, and $v$ is the vector of nodal velocities. The damping factor can be automatically calculated by Abaqus based off of damping intensity or a user specified damping factor. The method done in this thesis was to use a user specified damping factor. As analysis proceeds through a step, local regions may go unstable causing the local velocities to increase resulting in an increase to the viscous forces. If no instability arises, viscous forces remain very small. To ensure that the viscous forces never dominate the analysis it is recommended to compare the total viscous forces in the model to the applied total forces. Having this ratio remain small is an indication that the viscous forces did not falsely affect the predicted results. 
APPENDIX B: RESULTS OF GAIT ANALYSIS

Table 10: CP at $0 \%$ of Gait.

\begin{tabular}{|c|c|c|c|c|c|c|c|c|}
\hline & \multicolumn{8}{|c|}{ 0\% } \\
\hline & \multicolumn{4}{|c|}{ Tibial Cartilage } & \multicolumn{4}{|c|}{ Femoral Cartilage } \\
\hline & \multicolumn{2}{|c|}{ Lateral } & \multicolumn{2}{|c|}{ Medial } & \multicolumn{2}{|c|}{ Lateral } & \multicolumn{2}{|c|}{ Medial } \\
\hline & $\begin{array}{c}\text { Max Press } \\
\text { (MPa) }\end{array}$ & $\begin{array}{l}\text { \% Change } \\
\text { from Healthy }\end{array}$ & $\begin{array}{c}\text { Max Press } \\
(\mathrm{MPa})\end{array}$ & $\begin{array}{l}\text { \% Change } \\
\text { from Healthy }\end{array}$ & $\begin{array}{c}\text { Max Press } \\
\text { (MPa) }\end{array}$ & $\begin{array}{l}\text { \% Change } \\
\text { from Healthy }\end{array}$ & $\begin{array}{c}\text { Max Press } \\
(\mathrm{MPa})\end{array}$ & $\begin{array}{l}\text { \% Change } \\
\text { from Healthy }\end{array}$ \\
\hline Healthy & 1.46 & N/A & 1.64 & N/A & 1.41 & N/A & 1.49 & N/A \\
\hline AM Injury & 1.47 & 0.19 & 1.64 & 0.11 & 1.41 & 0.18 & 1.50 & 0.28 \\
\hline AM Def & 1.44 & -1.89 & 1.58 & -3.52 & 1.39 & -1.38 & 1.53 & 2.03 \\
\hline PL Injury & 1.47 & 0.32 & 1.60 & -2.27 & 1.42 & 0.26 & 1.54 & 3.26 \\
\hline PL Def & 1.47 & 0.47 & 1.70 & 3.39 & 1.42 & 0.56 & 1.65 & 10.48 \\
\hline ACL Injury & 1.47 & 0.44 & 1.61 & -1.88 & 1.42 & 0.41 & 1.55 & 3.73 \\
\hline ACL Rupture & 1.44 & -1.45 & 1.66 & 1.03 & 1.40 & -0.77 & 1.62 & 8.17 \\
\hline ВРТВ & 1.47 & 0.70 & 1.67 & 1.84 & 1.42 & 0.68 & 1.62 & 8.44 \\
\hline
\end{tabular}


Table 11: CP at $5 \%$ of Gait.

\begin{tabular}{|c|c|c|c|c|c|c|c|c|}
\hline & \multicolumn{8}{|c|}{$5 \%$} \\
\hline & \multicolumn{4}{|c|}{ Tibial Cartilage } & \multicolumn{4}{|c|}{ Femoral Cartilage } \\
\hline & \multicolumn{2}{|c|}{ Lateral } & \multicolumn{2}{|c|}{ Medial } & \multicolumn{2}{|c|}{ Lateral } & \multicolumn{2}{|c|}{ Medial } \\
\hline & $\begin{array}{c}\text { Max Press } \\
\text { (MPa) }\end{array}$ & $\begin{array}{l}\text { \% Change } \\
\text { from Healthy }\end{array}$ & $\begin{array}{c}\text { Max Press } \\
\text { (MPa) }\end{array}$ & $\begin{array}{l}\text { \% Change } \\
\text { from Healthy }\end{array}$ & $\begin{array}{c}\text { Max Press } \\
\text { (MPa) }\end{array}$ & $\begin{array}{l}\text { \% Change } \\
\text { from Healthy }\end{array}$ & $\begin{array}{c}\text { Max Press } \\
\text { (MPa) }\end{array}$ & $\begin{array}{l}\text { \% Change } \\
\text { from Healthy }\end{array}$ \\
\hline Healthy & 4.05 & N/A & 3.30 & N/A & 3.76 & N/A & 2.83 & N/A \\
\hline AM Injury & 4.04 & -0.09 & 3.30 & 0.00 & 3.76 & -0.03 & 2.83 & -0.12 \\
\hline AM Def & 4.03 & -0.49 & 3.30 & -0.10 & 3.76 & -0.20 & 2.85 & 0.49 \\
\hline PL Injury & 4.06 & 0.28 & 3.30 & -0.06 & 3.77 & 0.25 & 2.85 & 0.71 \\
\hline PL Def & 4.13 & 2.05 & 3.37 & 2.07 & 3.82 & 1.52 & 2.93 & 3.56 \\
\hline ACL Injury & 4.07 & 0.51 & 3.32 & 0.37 & 3.78 & 0.44 & 2.86 & 0.85 \\
\hline ACL Rupture & 4.14 & 2.23 & 3.40 & 2.93 & 3.75 & -0.29 & 2.97 & 5.01 \\
\hline ВРТВ & 4.10 & 1.37 & 3.32 & 0.64 & 3.81 & 1.11 & 2.87 & 1.45 \\
\hline
\end{tabular}


Table 12: CP at $15 \%$ of Gait.

\begin{tabular}{|c|c|c|c|c|c|c|c|c|}
\hline & \multicolumn{8}{|c|}{$15 \%$} \\
\hline & \multicolumn{4}{|c|}{ Tibial Cartilage } & \multicolumn{4}{|c|}{ Femoral Cartilage } \\
\hline & \multicolumn{2}{|c|}{ Lateral } & \multicolumn{2}{|c|}{ Medial } & \multicolumn{2}{|c|}{ Lateral } & \multicolumn{2}{|c|}{ Medial } \\
\hline & $\begin{array}{c}\text { Max Press } \\
\text { (MPa) }\end{array}$ & $\begin{array}{l}\text { \% Change } \\
\text { from Healthy }\end{array}$ & $\begin{array}{c}\text { Max Press } \\
\text { (MPa) }\end{array}$ & $\begin{array}{l}\text { \% Change } \\
\text { from Healthy }\end{array}$ & $\begin{array}{c}\text { Max Press } \\
\text { (MPa) }\end{array}$ & $\begin{array}{l}\% \text { Change } \\
\text { from Healthy }\end{array}$ & $\begin{array}{c}\text { Max Press } \\
\text { (MPa) }\end{array}$ & $\begin{array}{l}\text { \% Change } \\
\text { from Healthy }\end{array}$ \\
\hline Healthy & 6.16 & $\mathrm{~N} / \mathrm{A}$ & 8.05 & N/A & 5.83 & $\mathrm{~N} / \mathrm{A}$ & 7.59 & N/A \\
\hline AM Injury & 6.16 & 0.01 & 8.05 & 0.03 & 5.84 & 0.06 & 7.59 & 0.02 \\
\hline AM Def & 6.19 & 0.43 & 8.03 & -0.24 & 5.87 & 0.63 & 7.58 & -0.16 \\
\hline PL Injury & 6.16 & -0.10 & 8.05 & -0.02 & 5.84 & 0.10 & 7.59 & -0.04 \\
\hline PL Def & 6.14 & -0.38 & 8.06 & 0.15 & 5.84 & 0.06 & 7.55 & -0.56 \\
\hline ACL Injury & 6.16 & -0.03 & 8.05 & -0.02 & 5.84 & 0.21 & 7.59 & -0.03 \\
\hline ACL Rupture & 6.20 & 0.65 & 8.07 & 0.26 & 5.88 & 0.82 & 7.58 & -0.08 \\
\hline ВРТВ & 6.10 & -1.00 & 8.03 & -0.22 & 5.79 & -0.69 & 7.52 & -0.95 \\
\hline
\end{tabular}


Table 13: CP at 30\% of Gait.

\begin{tabular}{|c|c|c|c|c|c|c|c|c|}
\hline & \multicolumn{8}{|c|}{$30 \%$} \\
\hline & \multicolumn{4}{|c|}{ Tibial Cartilage } & \multicolumn{4}{|c|}{ Femoral Cartilage } \\
\hline & \multicolumn{2}{|c|}{ Lateral } & \multicolumn{2}{|c|}{ Medial } & \multicolumn{2}{|c|}{ Lateral } & \multicolumn{2}{|c|}{ Medial } \\
\hline & $\begin{array}{c}\text { Max Press } \\
(\mathrm{MPa})\end{array}$ & $\begin{array}{l}\% \text { Change } \\
\text { from Healthy }\end{array}$ & $\begin{array}{c}\text { Max Press } \\
\text { (MPa) }\end{array}$ & $\begin{array}{l}\% \text { Change } \\
\text { from Healthy }\end{array}$ & $\begin{array}{c}\text { Max Press } \\
\text { (MPa) }\end{array}$ & $\begin{array}{l}\% \text { Change } \\
\text { from Healthy }\end{array}$ & $\begin{array}{l}\text { Max Press } \\
\text { (MPa) }\end{array}$ & $\begin{array}{l}\text { \% Change } \\
\text { from Healthy }\end{array}$ \\
\hline Healthy & 4.20 & $\mathrm{~N} / \mathrm{A}$ & 5.96 & $\mathrm{~N} / \mathrm{A}$ & 3.91 & $\mathrm{~N} / \mathrm{A}$ & 5.48 & $\mathrm{~N} / \mathrm{A}$ \\
\hline AM Injury & 4.19 & -0.22 & 5.98 & 0.29 & 3.93 & 0.41 & 5.47 & -0.28 \\
\hline AM Def & 4.25 & 1.24 & 6.00 & 0.68 & 4.02 & 2.84 & 5.53 & 0.84 \\
\hline PL Injury & 4.18 & -0.44 & 5.98 & 0.42 & 3.95 & 0.97 & 5.50 & 0.29 \\
\hline PL Def & 4.25 & 1.37 & 5.99 & 0.52 & 4.07 & 4.10 & 5.56 & 1.35 \\
\hline ACL Injury & 4.18 & -0.40 & 6.00 & 0.71 & 3.96 & 1.35 & 5.48 & 0.04 \\
\hline ACL Rupture & 4.34 & 3.48 & 6.11 & 2.47 & 4.18 & 6.94 & 5.61 & 2.33 \\
\hline ВРТВ & 4.21 & 0.34 & 6.01 & 0.89 & 4.00 & 2.43 & 5.53 & 0.95 \\
\hline
\end{tabular}


Table 14: CP at $46 \%$ of Gait.

\begin{tabular}{|c|c|c|c|c|c|c|c|c|}
\hline & \multicolumn{8}{|c|}{$46 \%$} \\
\hline & \multicolumn{4}{|c|}{ Tibial Cartilage } & \multicolumn{4}{|c|}{ Femoral Cartilage } \\
\hline & \multicolumn{2}{|c|}{ Lateral } & \multicolumn{2}{|c|}{ Medial } & \multicolumn{2}{|c|}{ Lateral } & \multicolumn{2}{|c|}{ Medial } \\
\hline & $\begin{array}{c}\text { Max Press } \\
\text { (MPa) }\end{array}$ & $\begin{array}{l}\% \text { Change } \\
\text { from Healthy }\end{array}$ & $\begin{array}{c}\text { Max Press } \\
\text { (MPa) }\end{array}$ & $\begin{array}{l}\% \text { Change } \\
\text { from Healthy }\end{array}$ & $\begin{array}{c}\text { Max Press } \\
\text { (MPa) }\end{array}$ & $\begin{array}{l}\text { \% Change } \\
\text { from Healthy }\end{array}$ & $\begin{array}{c}\text { Max Press } \\
\text { (MPa) }\end{array}$ & $\begin{array}{l}\% \text { Change } \\
\text { from Healthy }\end{array}$ \\
\hline Healthy & 7.24 & N/A & 8.85 & N/A & 7.03 & N/A & 8.81 & N/A \\
\hline AM Injury & 7.26 & 0.31 & 8.89 & 0.49 & 7.05 & 0.23 & 8.86 & 0.60 \\
\hline AM Def & 7.44 & 2.85 & 9.07 & 2.46 & 7.19 & 2.30 & 9.10 & 3.32 \\
\hline PL Injury & 7.27 & 0.45 & 8.93 & 0.90 & 7.05 & 0.25 & 8.89 & 0.90 \\
\hline PL Def & 7.44 & 2.85 & 9.23 & 4.34 & 7.14 & 1.52 & 9.17 & 4.13 \\
\hline ACL Injury & 7.29 & 0.70 & 8.95 & 1.15 & 7.06 & 0.40 & 8.94 & 1.44 \\
\hline ACL Rupture & 7.66 & 5.91 & 9.54 & 7.82 & 7.31 & 3.90 & 9.37 & 6.35 \\
\hline ВРТВ & 7.39 & 2.17 & 9.11 & 2.93 & 7.10 & 0.96 & 9.14 & 3.77 \\
\hline
\end{tabular}


Table 15: CP at $60 \%$ of Gait.

\begin{tabular}{|c|c|c|c|c|c|c|c|c|}
\hline & \multicolumn{8}{|c|}{$60 \%$} \\
\hline & \multicolumn{4}{|c|}{ Tibial Cartilage } & \multicolumn{4}{|c|}{ Femoral Cartilage } \\
\hline & \multicolumn{2}{|c|}{ Lateral } & \multicolumn{2}{|c|}{ Medial } & \multicolumn{2}{|c|}{ Lateral } & \multicolumn{2}{|c|}{ Medial } \\
\hline & $\begin{array}{c}\text { Max Press } \\
\text { (MPa) }\end{array}$ & $\begin{array}{l}\text { \% Change } \\
\text { from Healthy }\end{array}$ & $\begin{array}{c}\text { Max Press } \\
\text { (MPa) }\end{array}$ & $\begin{array}{l}\text { \% Change } \\
\text { from Healthy }\end{array}$ & $\begin{array}{c}\text { Max Press } \\
\text { (MPa) }\end{array}$ & $\begin{array}{l}\text { \% Change } \\
\text { from Healthy }\end{array}$ & $\begin{array}{c}\text { Max Press } \\
\text { (MPa) }\end{array}$ & $\begin{array}{l}\% \text { Change } \\
\text { from Healthy }\end{array}$ \\
\hline Healthy & 3.63 & N/A & 1.30 & N/A & 3.29 & N/A & 1.92 & N/A \\
\hline AM Injury & 3.62 & -0.08 & 1.48 & 14.28 & 3.29 & 0.02 & 1.91 & -0.56 \\
\hline AM Def & 3.61 & -0.47 & 1.66 & 27.93 & 3.29 & -0.15 & 1.71 & -10.60 \\
\hline PL Injury & 3.63 & 0.08 & 1.46 & 12.11 & 3.30 & 0.19 & 1.96 & 2.14 \\
\hline PL Def & 3.62 & -0.20 & 1.49 & 14.78 & 3.30 & 0.23 & 2.05 & 6.93 \\
\hline ACL Injury & 3.62 & -0.16 & 1.46 & 12.83 & 3.30 & 0.05 & 1.98 & 3.32 \\
\hline ACL Rupture & 3.64 & 0.39 & 1.41 & 8.99 & 3.33 & 0.97 & 2.43 & 26.82 \\
\hline ВРТВ & 3.61 & -0.41 & 1.56 & 20.10 & 3.30 & 0.03 & 1.82 & -4.86 \\
\hline
\end{tabular}


Table 16: CA at $0 \%$ of Gait.

\begin{tabular}{|c|c|c|c|c|c|c|c|c|}
\hline & \multicolumn{8}{|c|}{$0 \%$} \\
\hline & \multicolumn{4}{|c|}{ Tibial Cartilage } & \multicolumn{4}{|c|}{ Femoral Cartilage } \\
\hline & \multicolumn{2}{|c|}{ Lateral } & \multicolumn{2}{|c|}{ Medial } & \multicolumn{2}{|c|}{ Lateral } & \multicolumn{2}{|c|}{ Medial } \\
\hline & $\begin{array}{c}\text { Contact Area } \\
\qquad\left(\mathrm{mm}^{2}\right)\end{array}$ & $\begin{array}{l}\text { \% Change } \\
\text { from Healthy }\end{array}$ & $\begin{array}{c}\text { Contact Area } \\
\left(\mathrm{mm}^{2}\right)\end{array}$ & $\begin{array}{l}\% \text { Change } \\
\text { from Healthy }\end{array}$ & $\begin{array}{c}\text { Contact Area } \\
\left(\mathrm{mm}^{2}\right)\end{array}$ & $\begin{array}{l}\% \text { Change } \\
\text { from Healthy }\end{array}$ & $\begin{array}{c}\text { Contact Area } \\
\qquad\left(\mathrm{mm}^{2}\right)\end{array}$ & $\begin{array}{l}\% \text { Change } \\
\text { from Healthy }\end{array}$ \\
\hline Healthy & 328.66 & N/A & 232.98 & N/A & 330.03 & N/A & 206.71 & N/A \\
\hline AM Injury & 328.65 & 0.00 & 232.98 & 0.00 & 330.03 & 0.00 & 206.73 & 0.01 \\
\hline AM Def & 333.28 & 1.41 & 232.68 & -0.13 & 334.66 & 1.40 & 212.31 & 2.71 \\
\hline PL Injury & 329.61 & 0.29 & 231.63 & -0.58 & 330.97 & 0.28 & 207.75 & 0.50 \\
\hline PL Def & 325.88 & -0.84 & 226.22 & -2.90 & 331.19 & 0.35 & 205.26 & -0.70 \\
\hline ACL Injury & 329.59 & 0.28 & 231.63 & -0.58 & 328.74 & -0.39 & 207.78 & 0.52 \\
\hline ACL Rupture & 333.50 & 1.47 & 228.00 & -2.14 & 340.80 & 3.26 & 213.90 & 3.48 \\
\hline ВРТВ & 327.57 & -0.33 & 229.68 & -1.42 & 331.86 & 0.55 & 206.55 & -0.08 \\
\hline
\end{tabular}


Table 17: CA at $5 \%$ of Gait.

\begin{tabular}{|c|c|c|c|c|c|c|c|c|}
\hline & \multicolumn{8}{|c|}{$5 \%$} \\
\hline & \multicolumn{4}{|c|}{ Tibial Cartilage } & \multicolumn{4}{|c|}{ Femoral Cartilage } \\
\hline & \multicolumn{2}{|c|}{ Lateral } & \multicolumn{2}{|c|}{ Medial } & \multicolumn{2}{|c|}{ Lateral } & \multicolumn{2}{|c|}{ Medial } \\
\hline & $\begin{array}{c}\text { Contact Area } \\
\qquad\left(\mathrm{mm}^{2}\right)\end{array}$ & $\begin{array}{l}\% \text { Change } \\
\text { from Healthy }\end{array}$ & $\begin{array}{c}\text { Contact Area } \\
\left(\mathrm{mm}^{2}\right)\end{array}$ & $\begin{array}{l}\% \text { Change } \\
\text { from Healthy }\end{array}$ & $\begin{array}{l}\text { Contact Area } \\
\qquad\left(\mathrm{mm}^{2}\right)\end{array}$ & $\begin{array}{l}\% \text { Change } \\
\text { from Healthy }\end{array}$ & $\begin{array}{l}\text { Contact Area } \\
\qquad\left(\mathrm{mm}^{2}\right)\end{array}$ & $\begin{array}{l}\% \text { Change } \\
\text { from Healthy }\end{array}$ \\
\hline Healthy & 386.11 & N/A & 248.42 & N/A & 322.61 & N/A & 194.95 & N/A \\
\hline AM Injury & 386.55 & 0.11 & 248.09 & -0.13 & 323.05 & 0.14 & 195.41 & 0.24 \\
\hline AM Def & 392.29 & 1.60 & 252.49 & 1.64 & 327.45 & 1.50 & 199.37 & 2.27 \\
\hline PL Injury & 384.11 & -0.52 & 251.74 & 1.33 & 322.75 & 0.04 & 198.27 & 1.71 \\
\hline PL Def & 377.67 & -2.19 & 251.54 & 1.26 & 321.76 & -0.27 & 201.76 & 3.49 \\
\hline ACL Injury & 379.86 & -1.62 & 247.61 & -0.33 & 319.32 & -1.02 & 194.92 & -0.02 \\
\hline ACL Rupture & 381.13 & -1.29 & 259.12 & 4.31 & 325.66 & 0.94 & 207.56 & 6.47 \\
\hline ВРТВ & 379.45 & -1.73 & 251.40 & 1.20 & 321.60 & -0.31 & 199.57 & 2.37 \\
\hline
\end{tabular}


Table 18: CA at $15 \%$ of Gait.

\begin{tabular}{|c|c|c|c|c|c|c|c|c|}
\hline & \multicolumn{8}{|c|}{$15 \%$} \\
\hline & \multicolumn{4}{|c|}{ Tibial Cartilage } & \multicolumn{4}{|c|}{ Femoral Cartilage } \\
\hline & \multicolumn{2}{|c|}{ Lateral } & \multicolumn{2}{|c|}{ Medial } & \multicolumn{2}{|c|}{ Lateral } & \multicolumn{2}{|c|}{ Medial } \\
\hline & $\begin{array}{l}\text { Contact Area } \\
\qquad\left(\mathrm{mm}^{2}\right)\end{array}$ & $\begin{array}{l}\% \text { Change } \\
\text { from Healthy }\end{array}$ & $\begin{array}{l}\text { Contact Area } \\
\qquad\left(\mathrm{mm}^{2}\right)\end{array}$ & $\begin{array}{l}\% \text { Change } \\
\text { from Healthy }\end{array}$ & $\begin{array}{c}\text { Contact Area } \\
\qquad\left(\mathrm{mm}^{2}\right)\end{array}$ & $\begin{array}{l}\% \text { Change } \\
\text { from Healthy }\end{array}$ & $\begin{array}{c}\text { Contact Area } \\
\qquad\left(\mathrm{mm}^{2}\right)\end{array}$ & $\begin{array}{l}\text { \% Change } \\
\text { from Healthy }\end{array}$ \\
\hline Healthy & 285.64 & N/A & 336.79 & N/A & 243.44 & N/A & 313.14 & N/A \\
\hline AM Injury & 284.44 & -0.42 & 338.23 & 0.43 & 243.47 & 0.01 & 312.94 & -0.06 \\
\hline AM Def & 284.79 & -0.30 & 344.82 & 2.39 & 243.07 & -0.15 & 319.32 & 1.97 \\
\hline PL Injury & 283.05 & -0.91 & 338.27 & 0.44 & 241.35 & -0.86 & 313.57 & 0.14 \\
\hline PL Def & 283.04 & -0.91 & 344.01 & 2.14 & 237.21 & -2.56 & 317.49 & 1.39 \\
\hline ACL Injury & 284.71 & -0.33 & 340.49 & 1.10 & 241.49 & -0.80 & 314.55 & 0.45 \\
\hline ACL Rupture & 284.74 & -0.32 & 356.16 & 5.75 & 239.25 & -1.72 & 323.81 & 3.41 \\
\hline ВРТВ & 285.85 & 0.07 & 340.66 & 1.15 & 246.83 & 1.40 & 314.47 & 0.42 \\
\hline
\end{tabular}


Table 19: CA at $30 \%$ of Gait.

\begin{tabular}{|c|c|c|c|c|c|c|c|c|}
\hline & \multicolumn{8}{|c|}{$30 \%$} \\
\hline & \multicolumn{4}{|c|}{ Tibial Cartilage } & \multicolumn{4}{|c|}{ Femoral Cartilage } \\
\hline & \multicolumn{2}{|c|}{ Lateral } & \multicolumn{2}{|c|}{ Medial } & \multicolumn{2}{|c|}{ Lateral } & \multicolumn{2}{|c|}{ Medial } \\
\hline & $\begin{array}{l}\text { Contact Area } \\
\qquad\left(\mathrm{mm}^{2}\right)\end{array}$ & $\begin{array}{l}\% \text { Change } \\
\text { from Healthy }\end{array}$ & $\begin{array}{c}\text { Contact Area } \\
\left(\mathrm{mm}^{2}\right)\end{array}$ & $\begin{array}{l}\% \text { Change } \\
\text { from Healthy }\end{array}$ & $\begin{array}{c}\text { Contact Area } \\
\left(\mathrm{mm}^{2}\right)\end{array}$ & $\begin{array}{l}\% \text { Change } \\
\text { from Healthy }\end{array}$ & $\begin{array}{c}\text { Contact Area } \\
\left(\mathrm{mm}^{2}\right)\end{array}$ & $\begin{array}{l}\text { \% Change } \\
\text { from Healthy }\end{array}$ \\
\hline Healthy & 213.06 & N/A & 310.16 & N/A & 183.32 & N/A & 263.74 & N/A \\
\hline AM Injury & 212.60 & -0.21 & 310.46 & 0.10 & 183.26 & -0.03 & 264.38 & 0.24 \\
\hline AM Def & 209.78 & -1.54 & 315.55 & 1.74 & 183.20 & -0.07 & 269.84 & 2.31 \\
\hline PL Injury & 212.39 & -0.32 & 310.25 & 0.03 & 182.41 & -0.50 & 263.66 & -0.03 \\
\hline PL Def & 208.71 & -2.04 & 298.26 & -3.84 & 175.50 & -4.27 & 254.80 & -3.39 \\
\hline ACL Injury & 210.77 & -1.07 & 310.55 & 0.13 & 181.94 & -0.75 & 262.66 & -0.41 \\
\hline ACL Rupture & 203.10 & -4.67 & 301.80 & -2.70 & 178.68 & -2.53 & 254.26 & -3.59 \\
\hline BPTB & 208.67 & -2.06 & 304.29 & -1.89 & 176.44 & -3.75 & 259.86 & -1.47 \\
\hline
\end{tabular}


Table 20: CA at $46 \%$ of Gait.

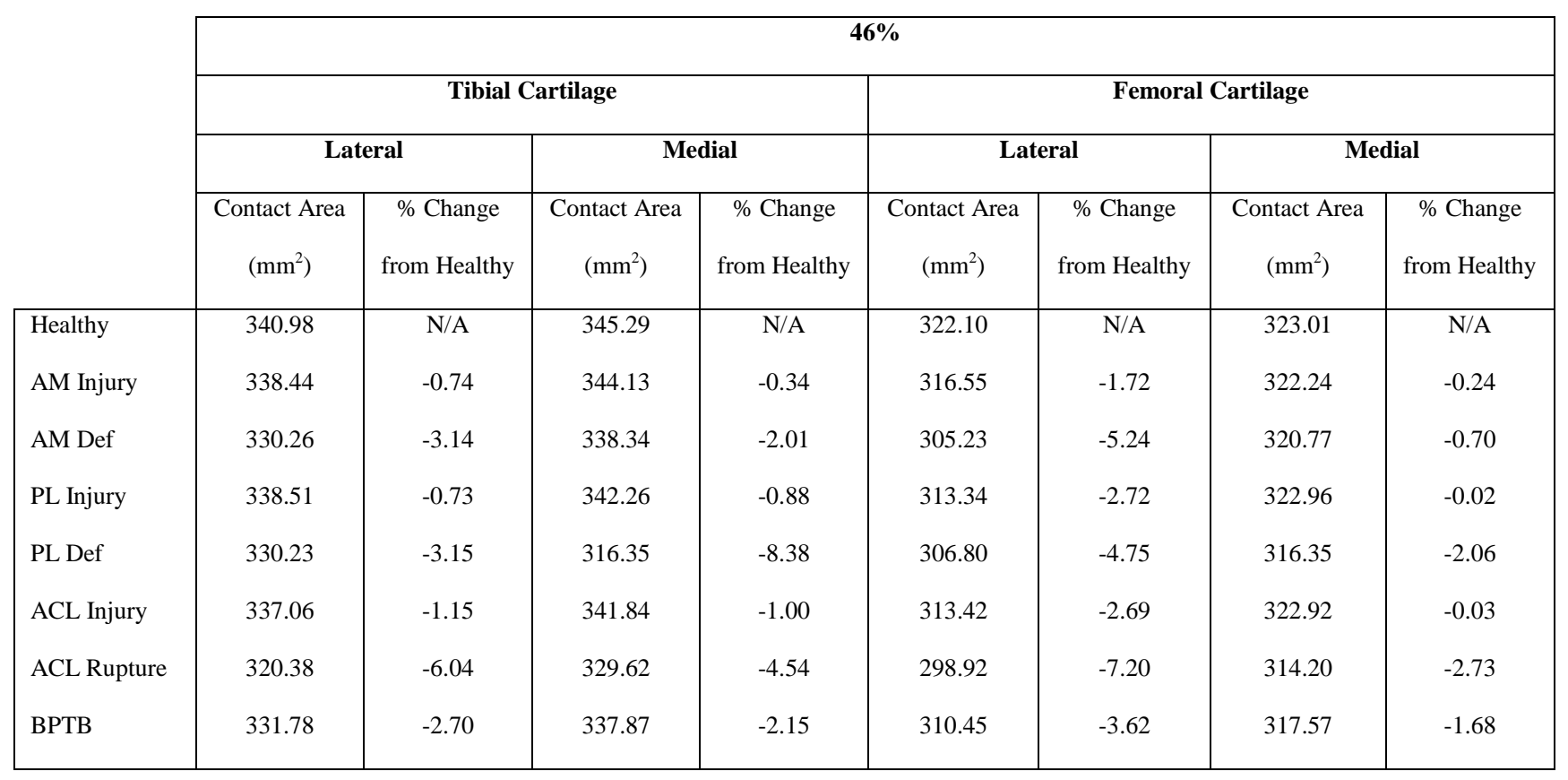


Table 21: CA at $60 \%$ of Gait.

\begin{tabular}{|c|c|c|c|c|c|c|c|c|}
\hline & \multicolumn{8}{|c|}{$60 \%$} \\
\hline & \multicolumn{4}{|c|}{ Tibial Cartilage } & \multicolumn{4}{|c|}{ Femoral Cartilage } \\
\hline & \multicolumn{2}{|c|}{ Lateral } & \multicolumn{2}{|c|}{ Medial } & \multicolumn{2}{|c|}{ Lateral } & \multicolumn{2}{|c|}{ Medial } \\
\hline & $\begin{array}{c}\text { Contact Area } \\
\left(\mathrm{mm}^{2}\right)\end{array}$ & $\begin{array}{l}\% \text { Change } \\
\text { from Healthy }\end{array}$ & $\begin{array}{c}\text { Contact Area } \\
\left(\mathrm{mm}^{2}\right)\end{array}$ & $\begin{array}{l}\% \text { Change } \\
\text { from Healthy }\end{array}$ & $\begin{array}{c}\text { Contact Area } \\
\left(\mathrm{mm}^{2}\right)\end{array}$ & $\begin{array}{l}\% \text { Change } \\
\text { from Healthy }\end{array}$ & $\begin{array}{c}\text { Contact } \\
\text { Area }\left(\mathrm{mm}^{2}\right)\end{array}$ & $\begin{array}{l}\% \text { Change } \\
\text { from Healthy }\end{array}$ \\
\hline Healthy & 218.78 & N/A & 44.78 & N/A & 191.18 & N/A & 35.76 & N/A \\
\hline AM Injury & 219.25 & 0.21 & 44.78 & 0.00 & 190.53 & -0.34 & 35.76 & 0.01 \\
\hline AM Def & 221.45 & 1.22 & 44.76 & -0.04 & 195.80 & 2.42 & 35.79 & 0.08 \\
\hline PL Injury & 217.94 & -0.38 & 42.28 & -5.57 & 189.30 & -0.98 & 36.86 & 3.08 \\
\hline PL Def & 212.11 & -3.05 & 38.75 & -13.46 & 190.86 & -0.16 & 38.75 & 8.36 \\
\hline ACL Injury & 217.27 & -0.69 & 44.78 & 0.01 & 188.65 & -1.32 & 36.86 & 3.08 \\
\hline ACL Rupture & 213.76 & -2.30 & 45.28 & 1.13 & 198.68 & 3.93 & 40.00 & 11.88 \\
\hline ВРТВ & 214.99 & -1.73 & 42.28 & -5.58 & 187.04 & -2.17 & 35.76 & 0.01 \\
\hline
\end{tabular}


Table 22: ATT during Gait.

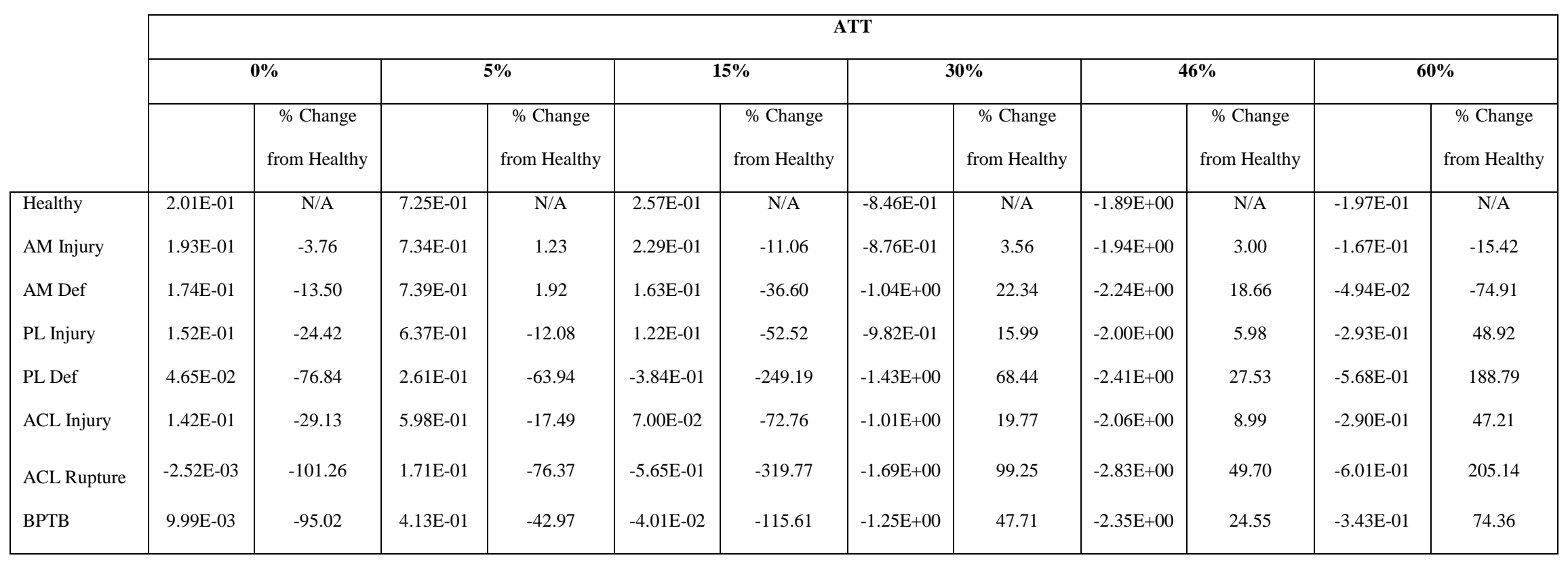


Table 23: Viscous Forces to Total Forces Ratio.

\begin{tabular}{|l|c|c|c|c|c|c|}
\cline { 2 - 7 } \multicolumn{1}{c|}{} & $\mathbf{0} \%$ & $\mathbf{5 \%}$ & $\mathbf{1 5 \%}$ & $\mathbf{3 0} \%$ & $\mathbf{4 6} \%$ & $\mathbf{6 0} \%$ \\
\hline Healthy & $4.53 \mathrm{E}-03$ & $8.16 \mathrm{E}-03$ & $2.50 \mathrm{E}-03$ & $7.85 \mathrm{E}-03$ & $8.44 \mathrm{E}-03$ & $1.67 \mathrm{E}-02$ \\
AM Injury & $4.60 \mathrm{E}-03$ & $8.15 \mathrm{E}-03$ & $2.40 \mathrm{E}-03$ & $8.12 \mathrm{E}-03$ & $8.61 \mathrm{E}-03$ & $1.69 \mathrm{E}-02$ \\
PL Def & $4.36 \mathrm{E}-03$ & $8.15 \mathrm{E}-03$ & $2.31 \mathrm{E}-03$ & $9.41 \mathrm{E}-03$ & $9.55 \mathrm{E}-03$ & $1.81 \mathrm{E}-02$ \\
PL Def & $4.25 \mathrm{E}-03$ & $8.14 \mathrm{E}-03$ & $2.40 \mathrm{E}-03$ & $8.10 \mathrm{E}-03$ & $1.46 \mathrm{E}-02$ & $1.71 \mathrm{E}-02$ \\
ACL Injury & $3.89 \mathrm{E}-03$ & $7.80 \mathrm{E}-03$ & $2.14 \mathrm{E}-03$ & $9.15 \mathrm{E}-03$ & $9.19 \mathrm{E}-03$ & $1.76 \mathrm{E}-02$ \\
ACL Rupture & $4.25 \mathrm{E}-03$ & $8.08 \mathrm{E}-03$ & $2.32 \mathrm{E}-03$ & $8.31 \mathrm{E}-03$ & $8.80 \mathrm{E}-03$ & $1.74 \mathrm{E}-02$ \\
BPTB & $3.71 \mathrm{E}-03$ & $7.69 \mathrm{E}-03$ & $2.39 \mathrm{E}-03$ & $1.09 \mathrm{E}-02$ & $1.05 \mathrm{E}-02$ & $2.01 \mathrm{E}-02$ \\
\hline
\end{tabular}




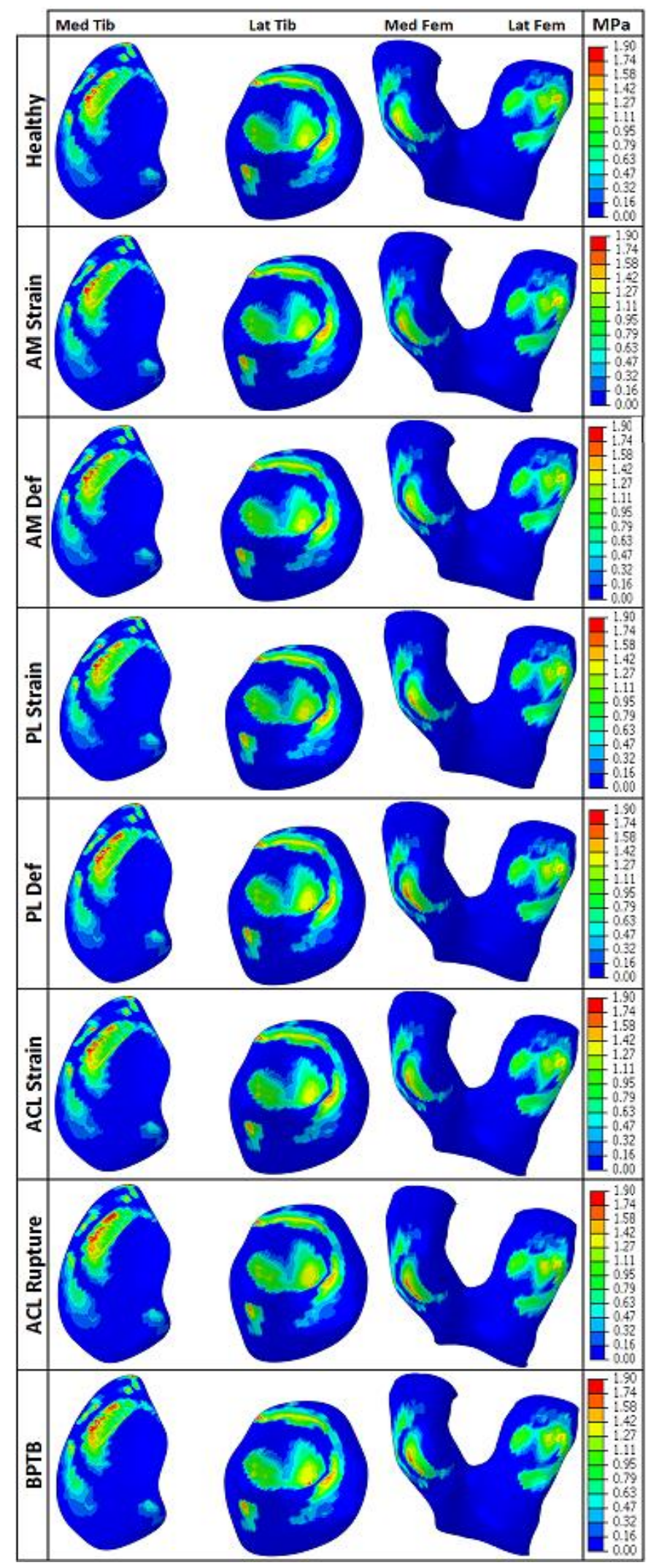

Figure 33: Articular cartilage contact pressure at $0 \%$ of gait. 


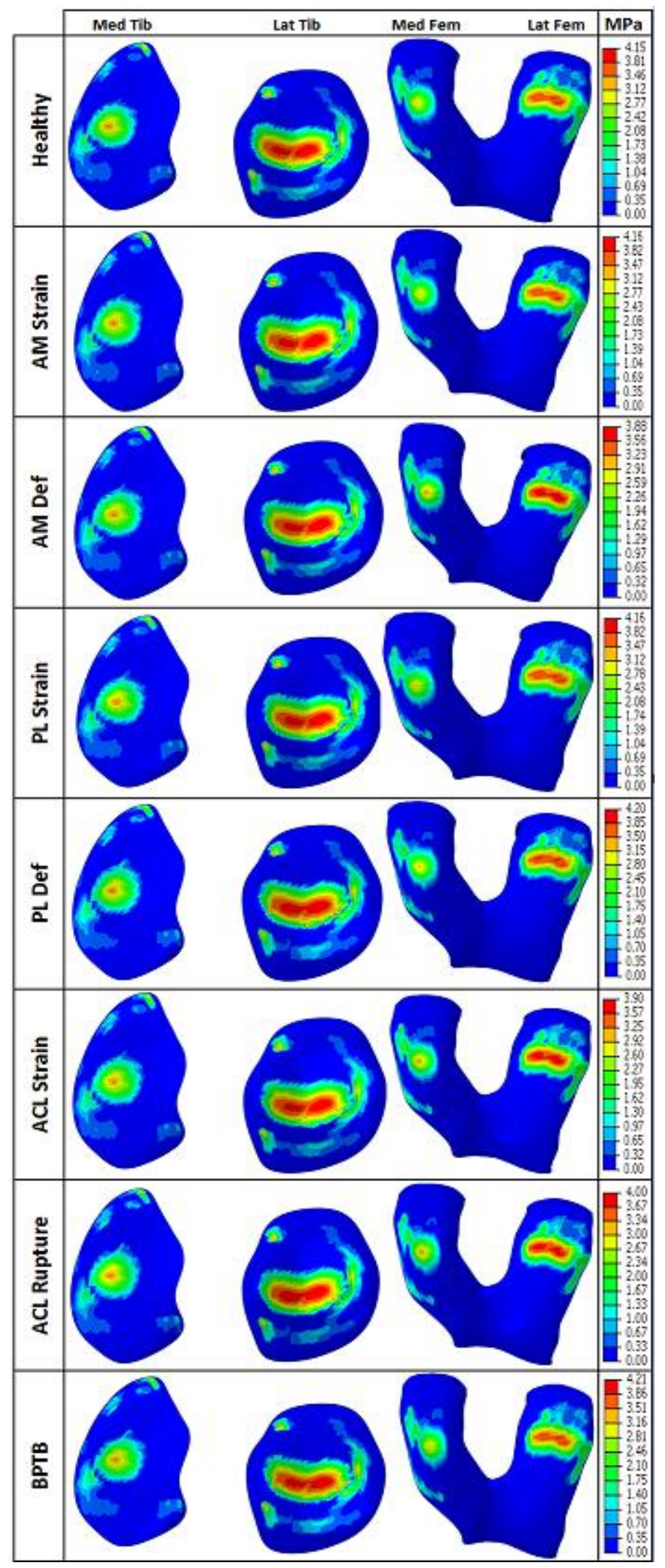

Figure 34: Articular cartilage contact pressure at $5 \%$ of Gait. 


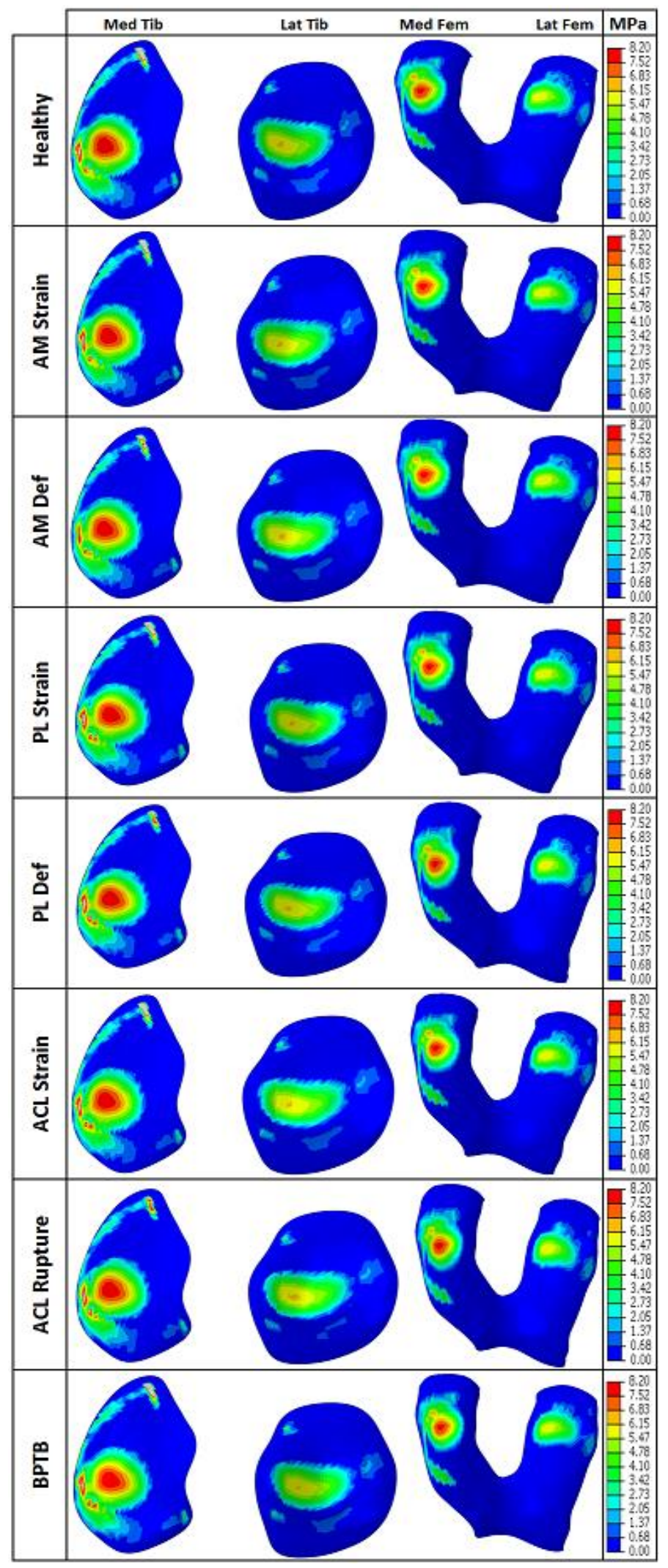

Figure 35: Articular cartilage contact pressure at $15 \%$ of Gait. 


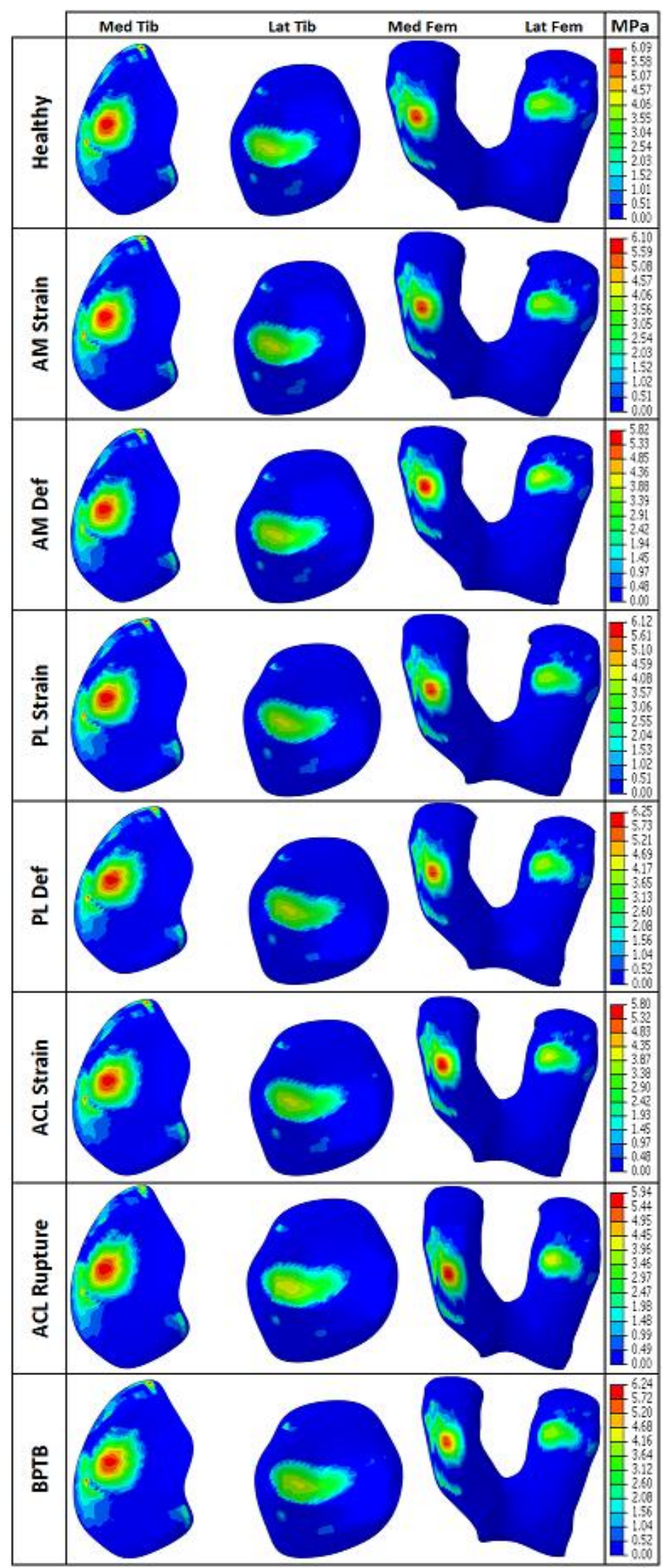

Figure 36: Articular cartilage contact pressure at $30 \%$ of Gait. 


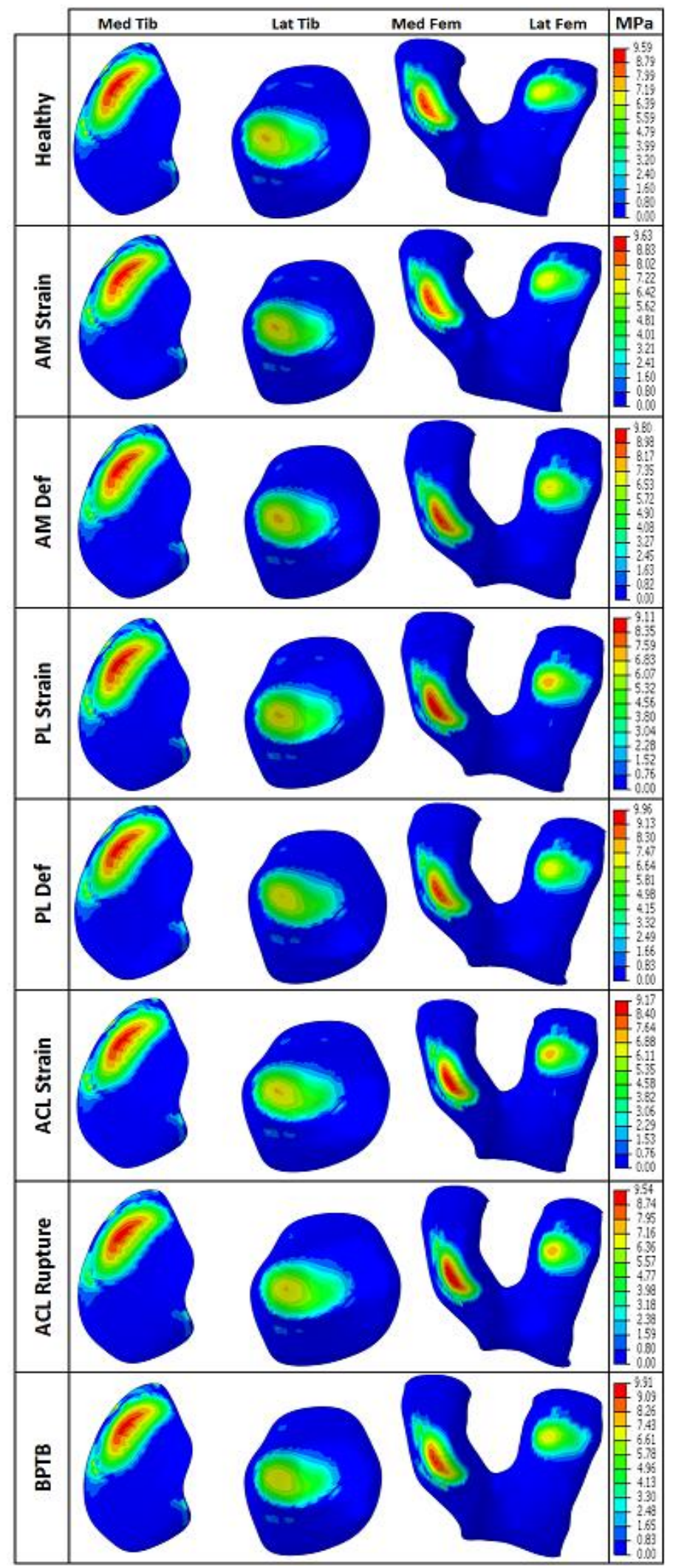

Figure 37: Articular cartilage contact pressure at $46 \%$ of Gait. 


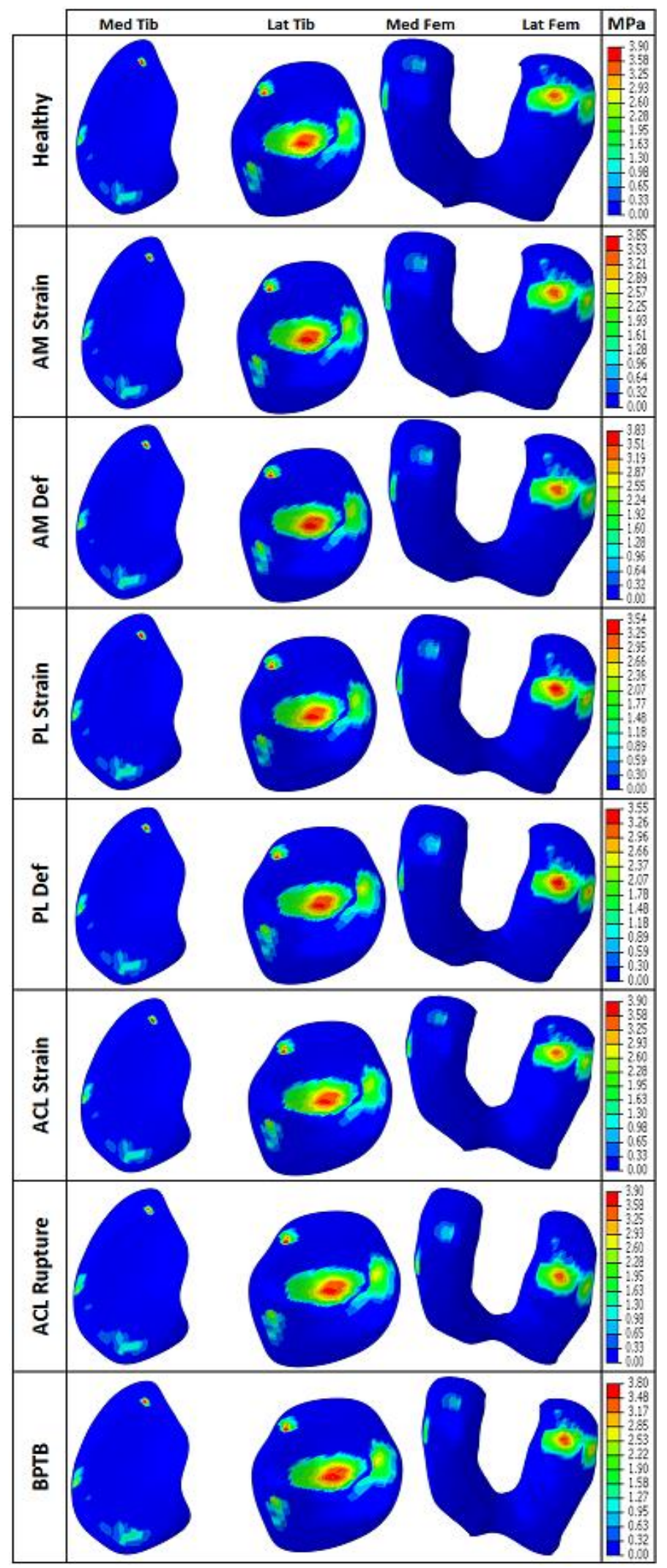

Figure 38: Articular cartilage contact pressure at $60 \%$ of Gait. 
APPENDIX C: CHANGES TO CP AND CA FOR AM DEFICIENT, PL DEFICIENT, ACL RUPTURED AND ACL RS KNEES THROUGHOUT GAIT

$0 \%$ of Gait

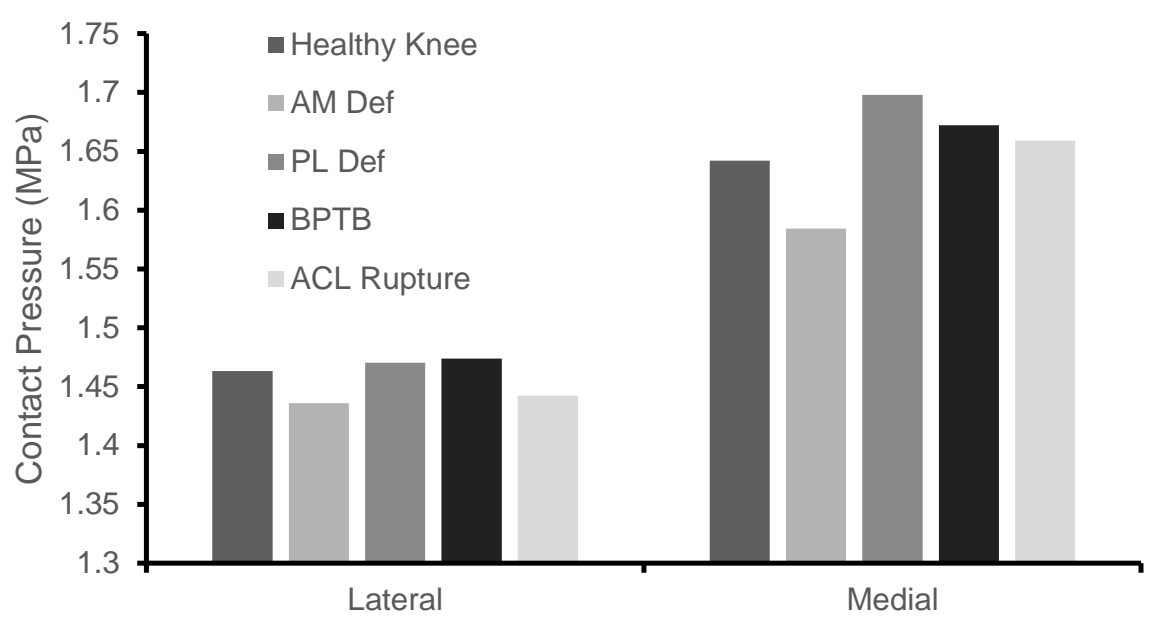

Figure 39: CP of tibial cartilage at $0 \%$ of Gait.

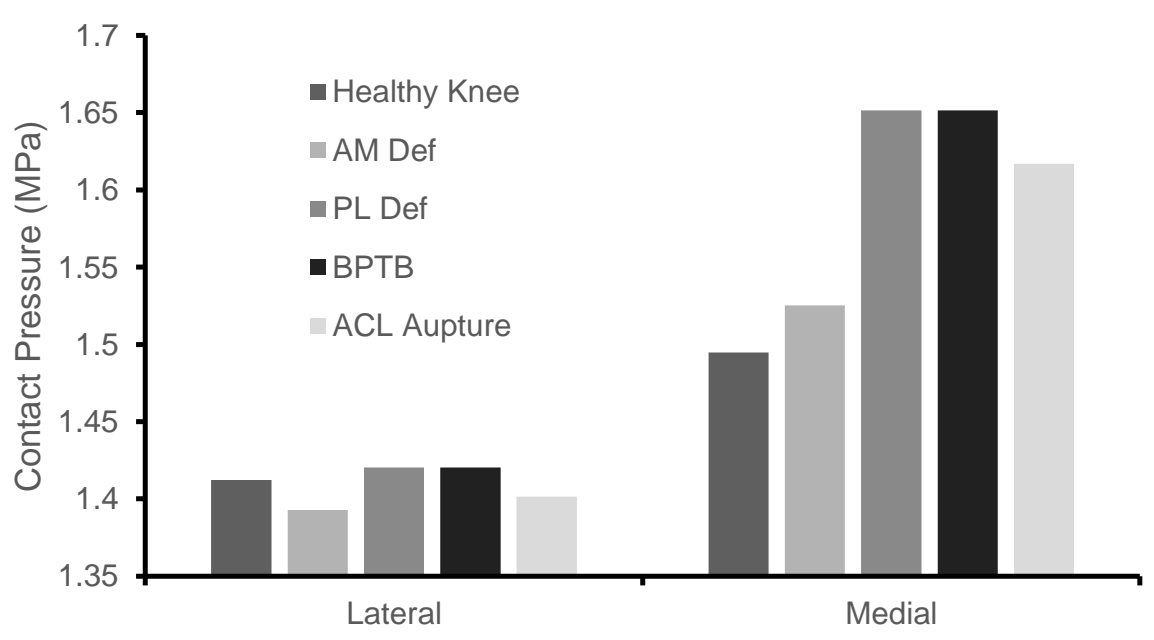

Figure 40: CP of femoral cartilage at $0 \%$ of Gait. 


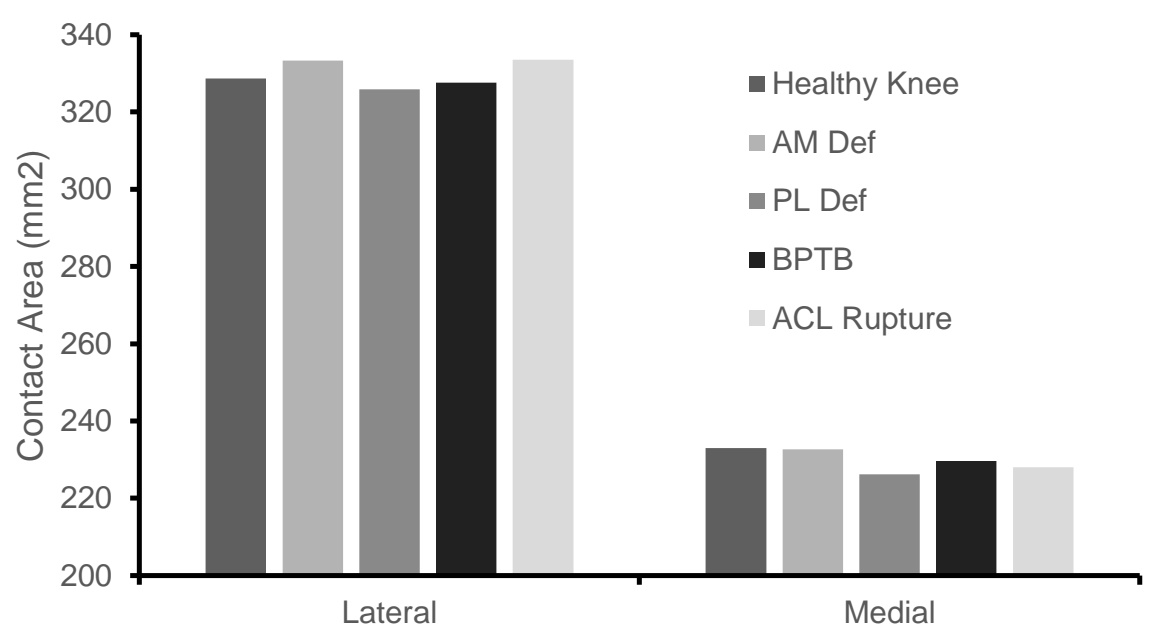

Figure 41 : CA of tibial cartilage at $0 \%$ of Gait.

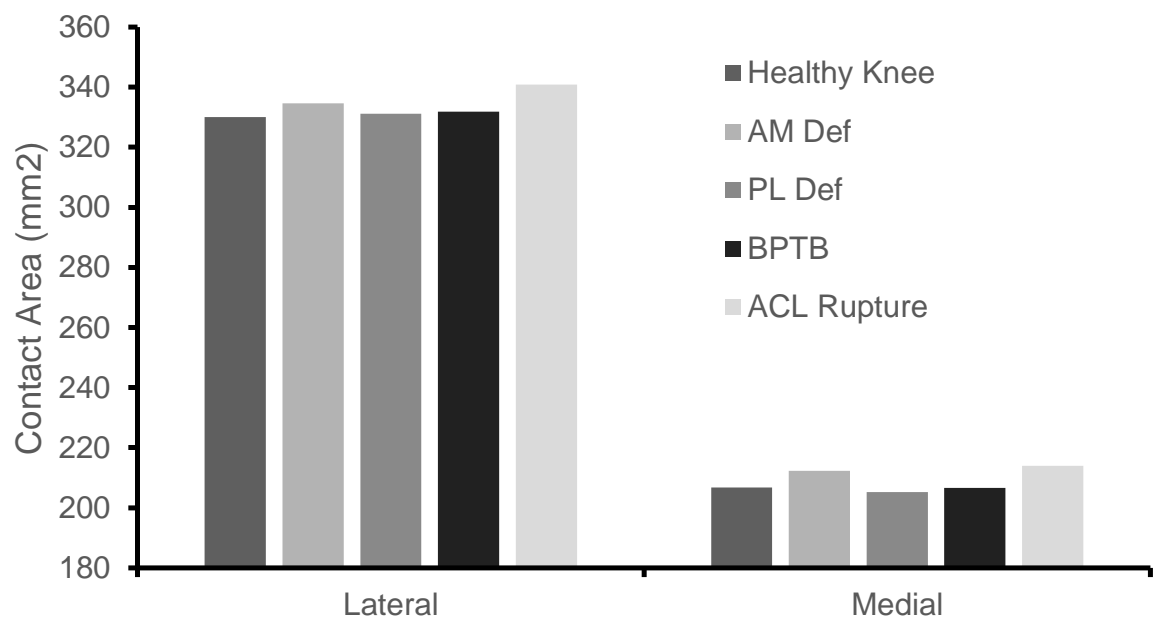

Figure 42 : CA of femoral cartilage at $0 \%$ of Gait. 
$5 \%$ of Gait

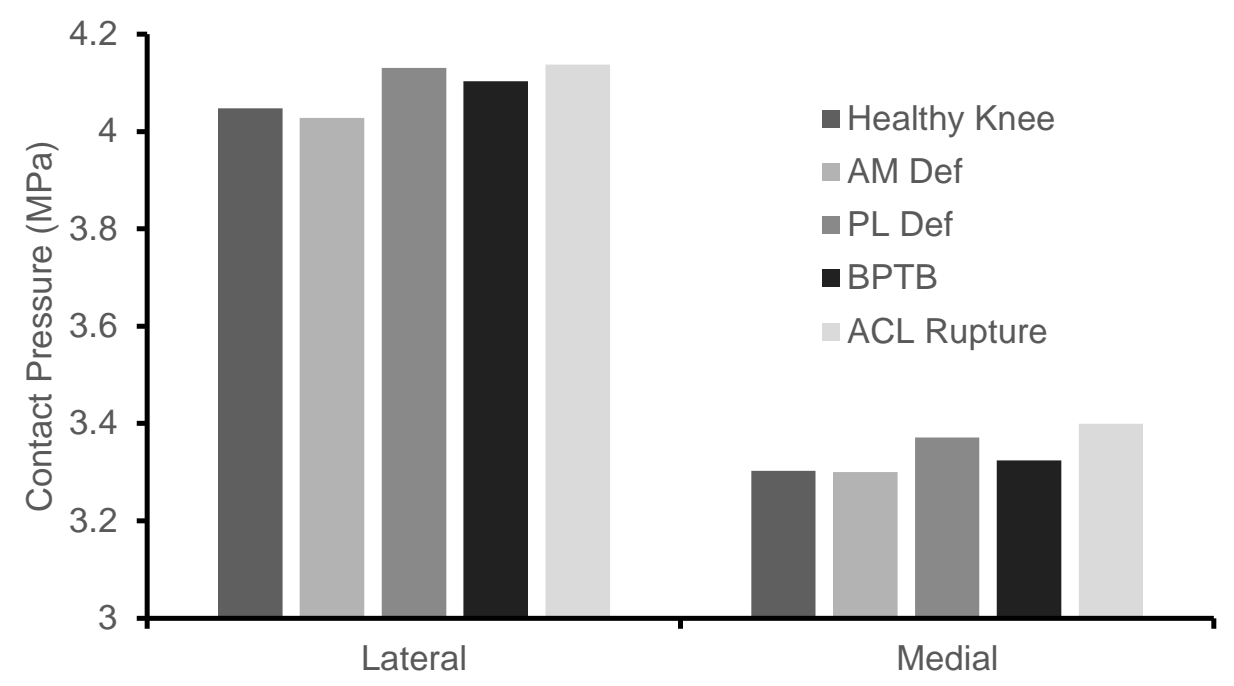

Figure 43: CP of tibial cartilage at $5 \%$ of Gait.

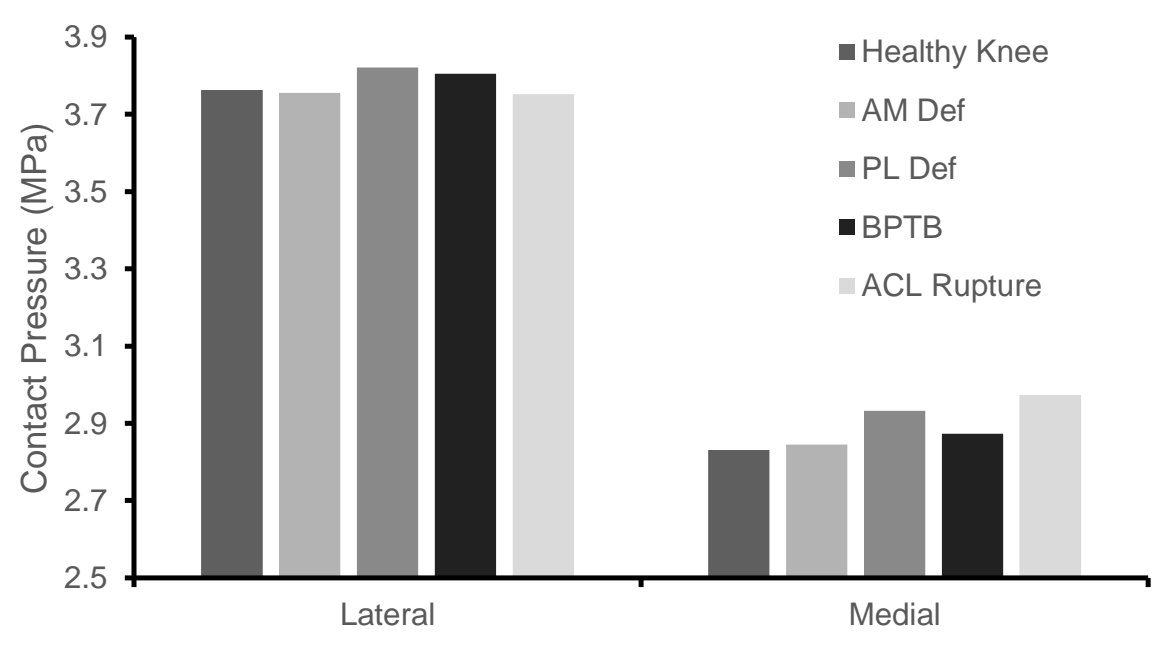

Figure 44: CP of femoral cartilage at $5 \%$ of Gait. 


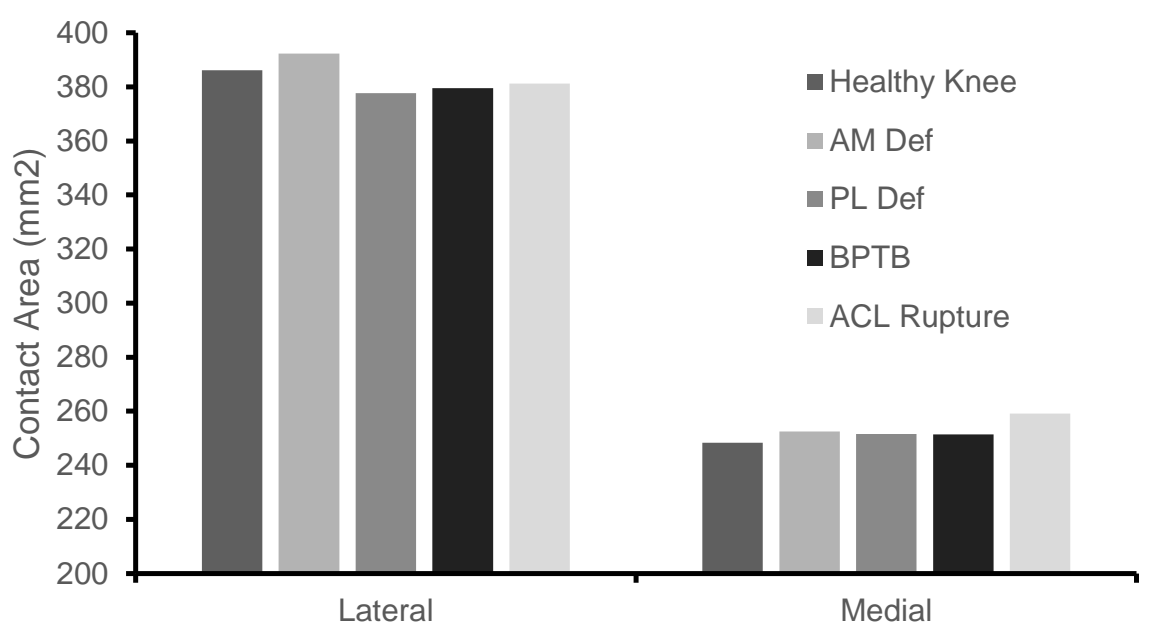

Figure 45: CA of tibial cartilage at $5 \%$ of Gait.

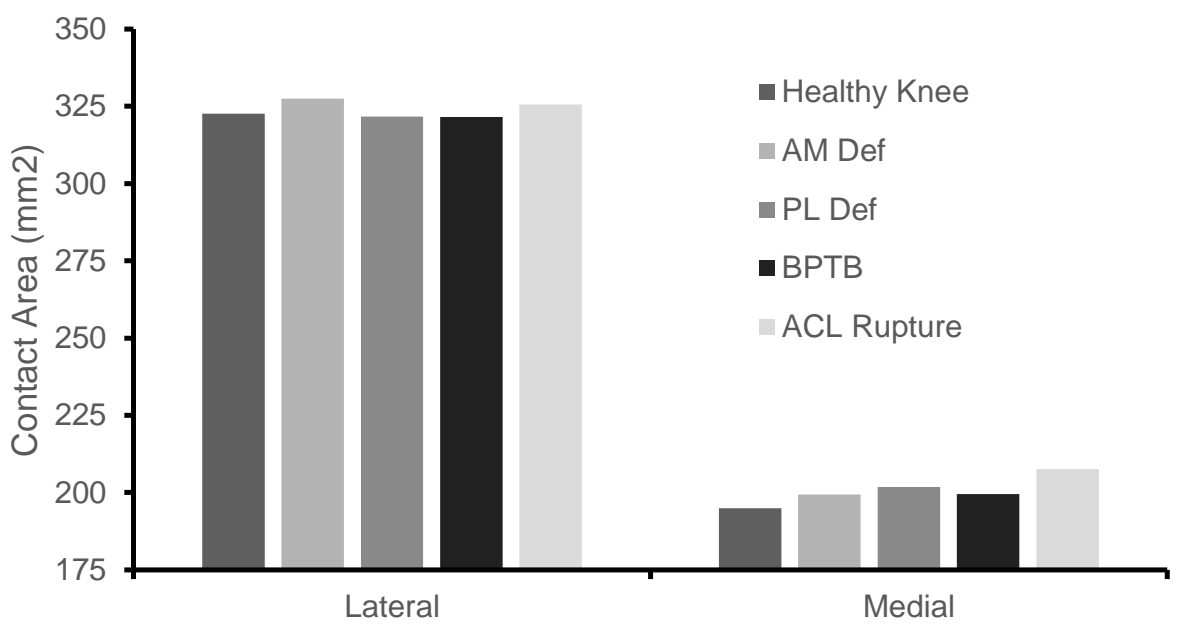

Figure 46: CA of femoral cartilage at $5 \%$ of Gait. 
$15 \%$ of Gait

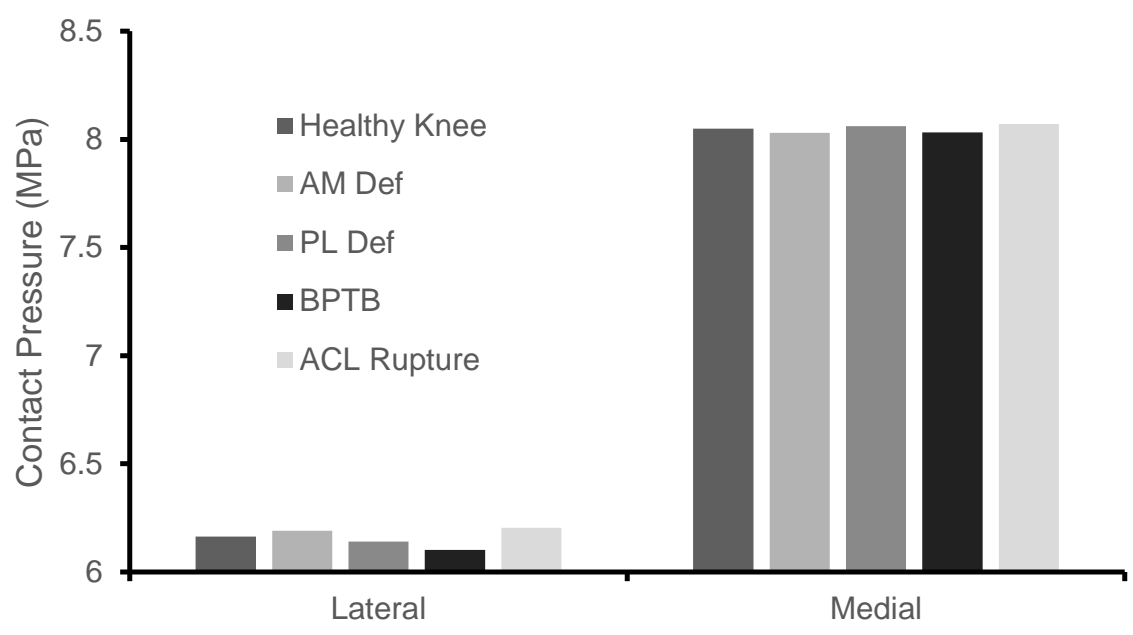

Figure 47: CP of tibial cartilage at $15 \%$ of Gait.

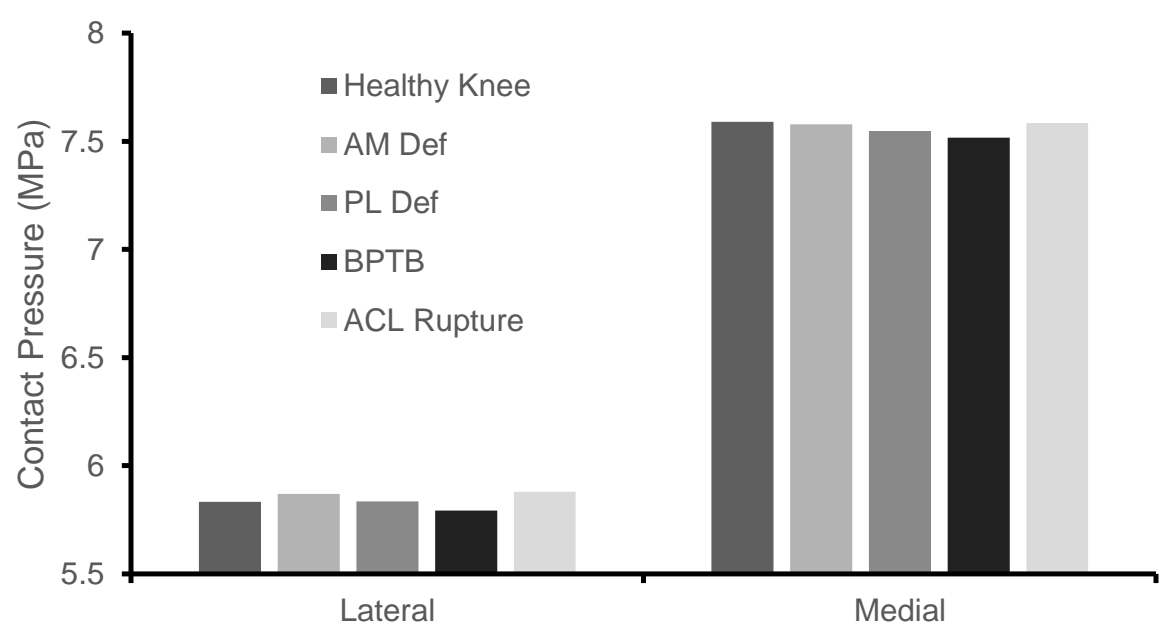

Figure 48: CP of femoral cartilage at $15 \%$ of Gait. 


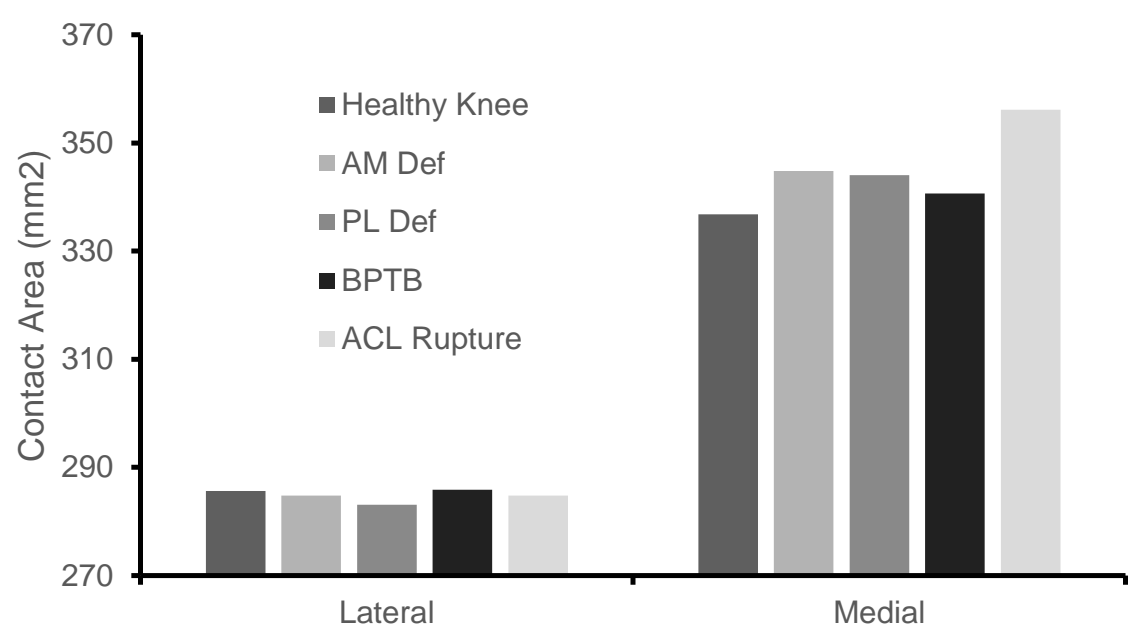

Figure 49: CA of tibial cartilage at $15 \%$ of Gait.

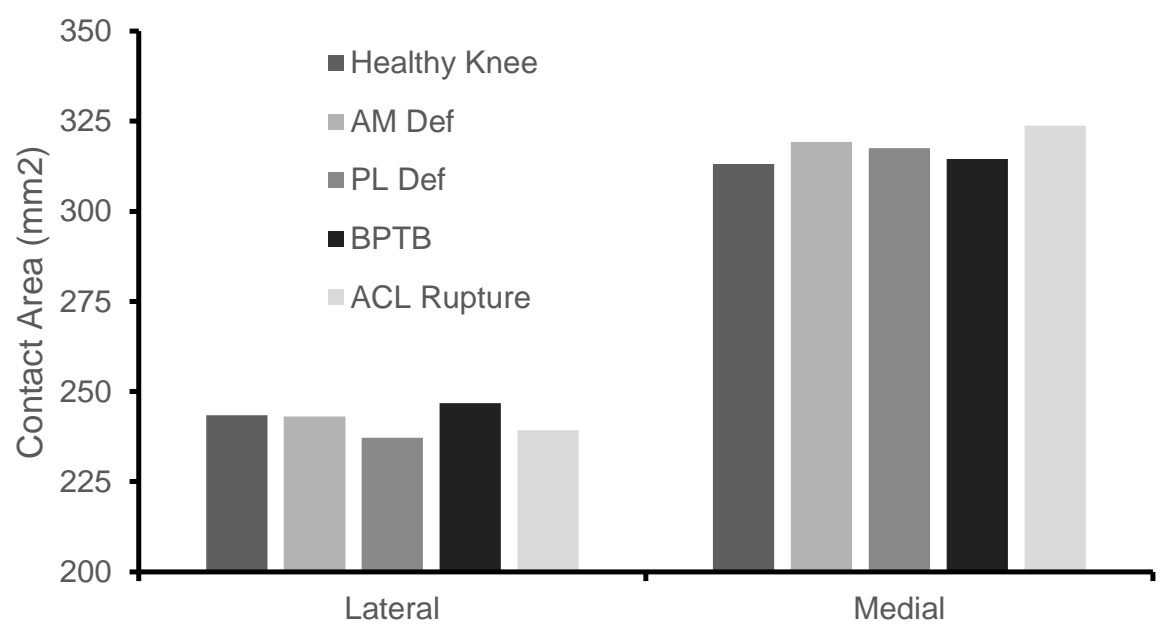

Figure 50: CA of femoral cartilage at $15 \%$ of Gait. 
$30 \%$ of Gait

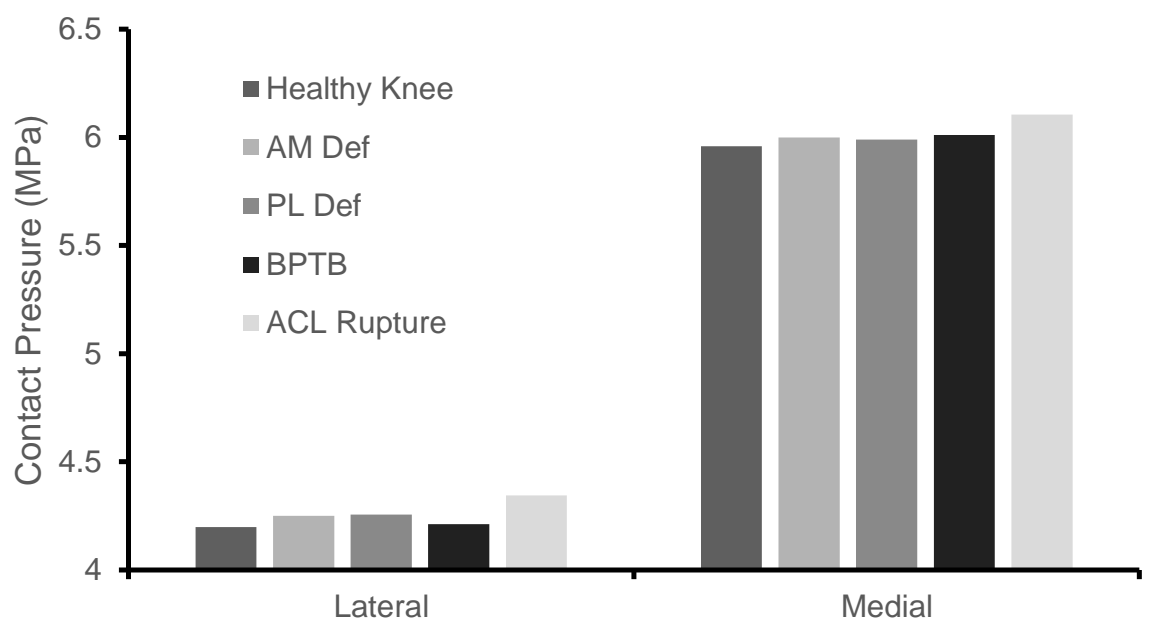

Figure 51: CP of tibial cartilage at $30 \%$ of Gait.

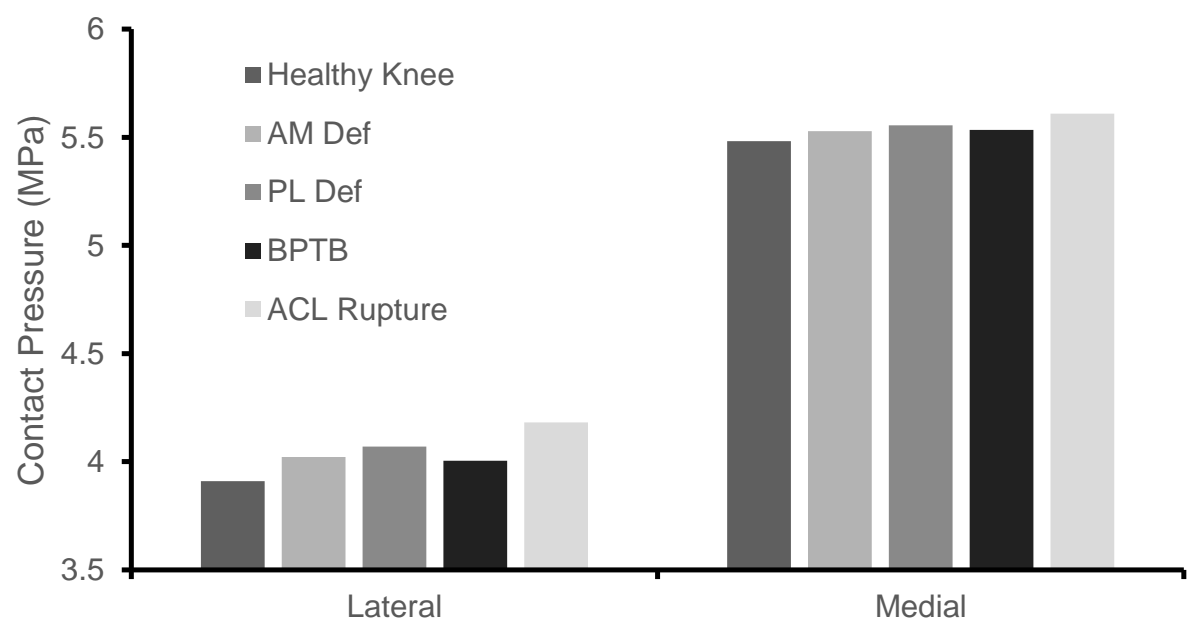

Figure 52: CP of femoral cartilage at $30 \%$ of Gait. 


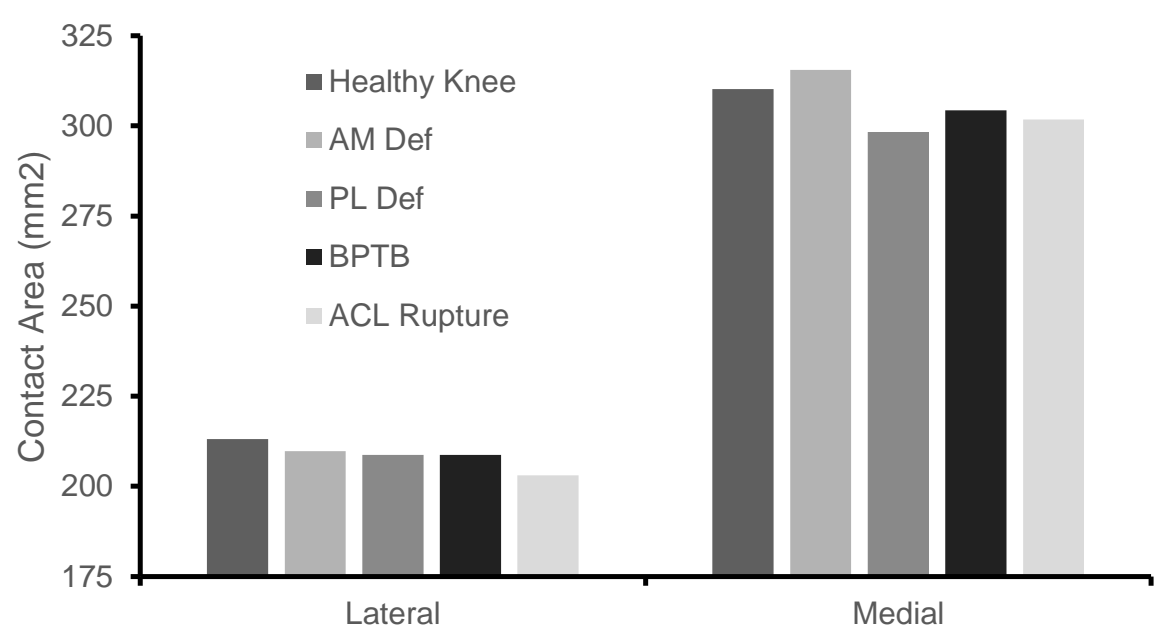

Figure 53: CA of tibial cartilage at $30 \%$ of Gait.

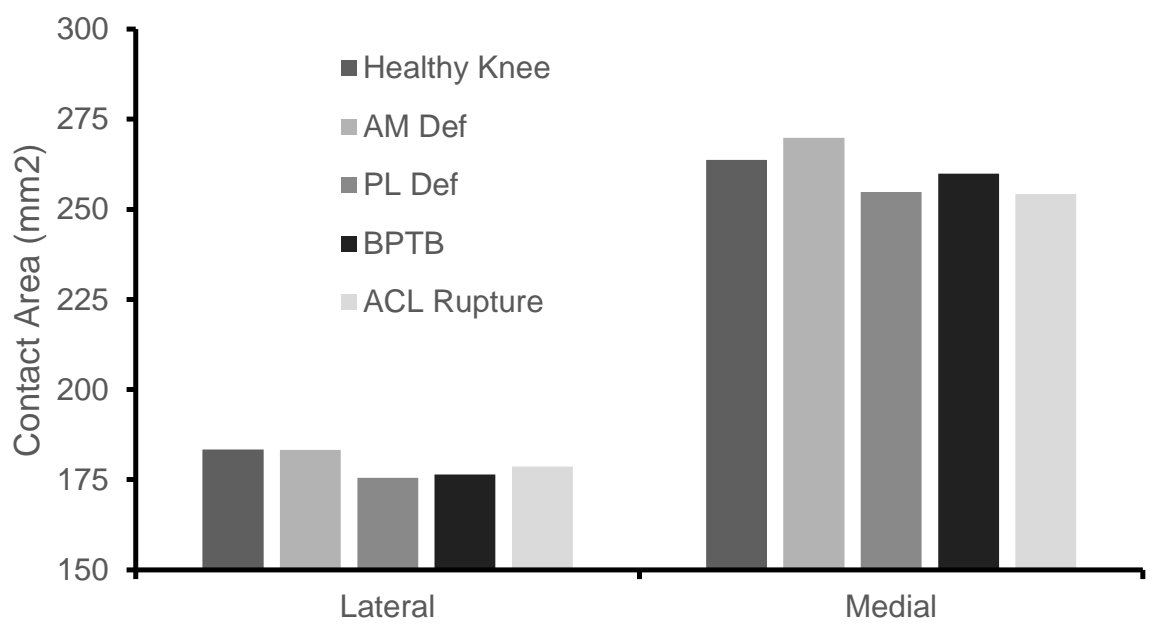

Figure 54: CA of femoral cartilage at $30 \%$ of Gait. 
$46 \%$ of Gait

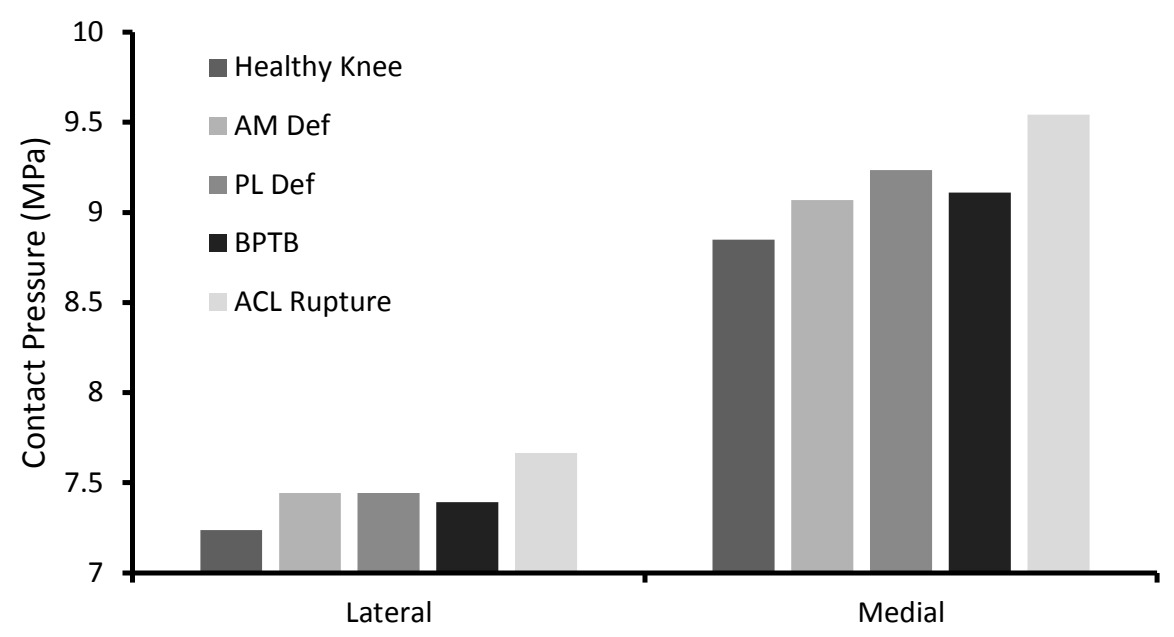

Figure 55: CP of tibial cartilage at $46 \%$ of Gait.

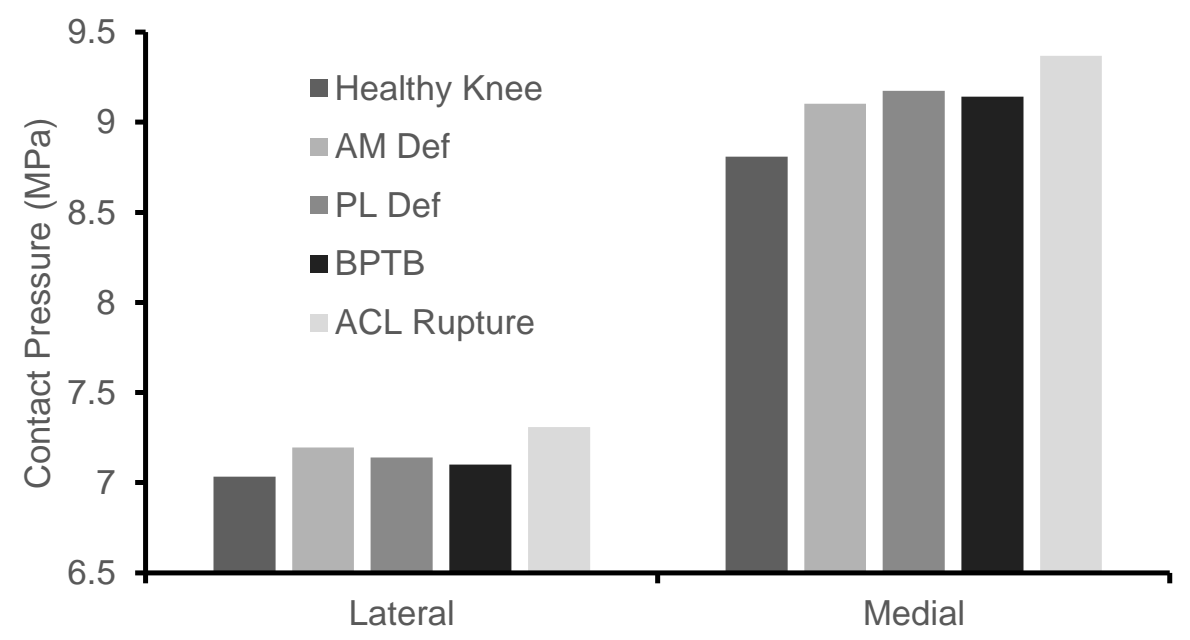

Figure 56: CP of femoral cartilage at $46 \%$ of Gait. 


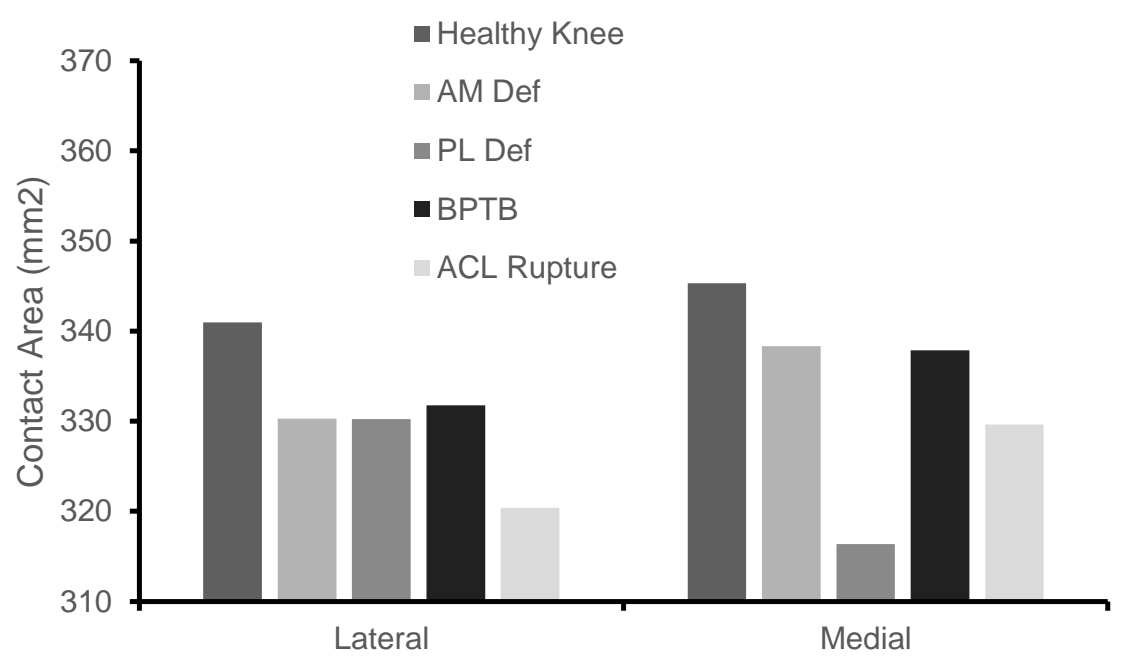

Figure 57: CA of tibial cartilage at $46 \%$ of Gait.

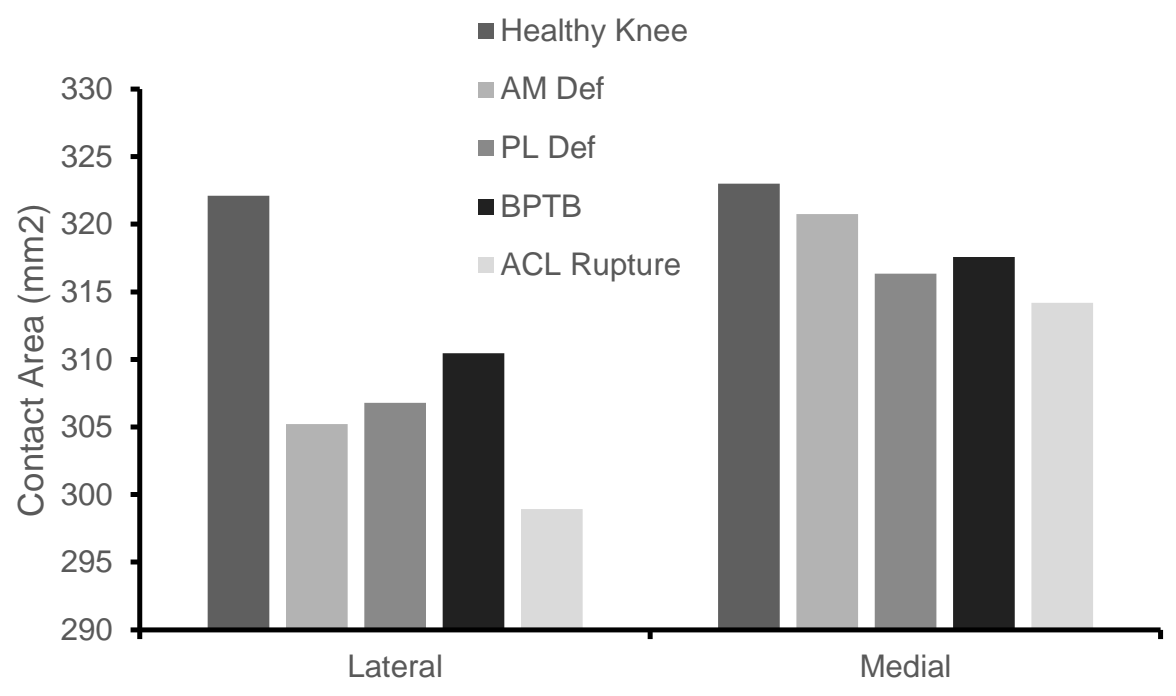

Figure 58: CA of femoral cartilage at $46 \%$ of Gait. 


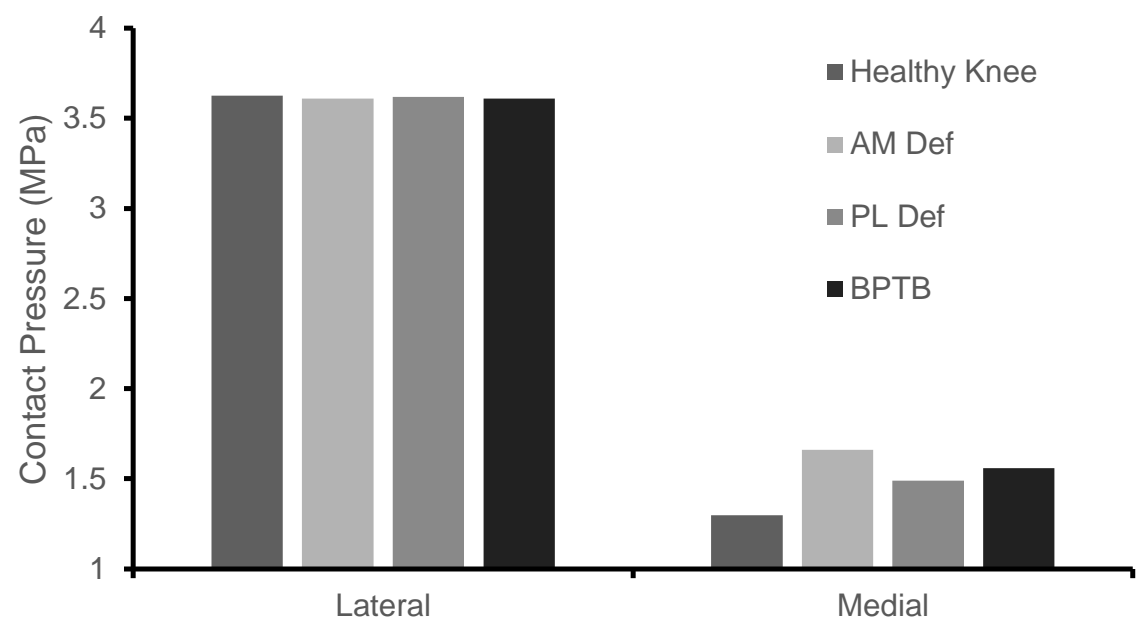

Figure 59: CP of tibial cartilage at $60 \%$ of Gait.

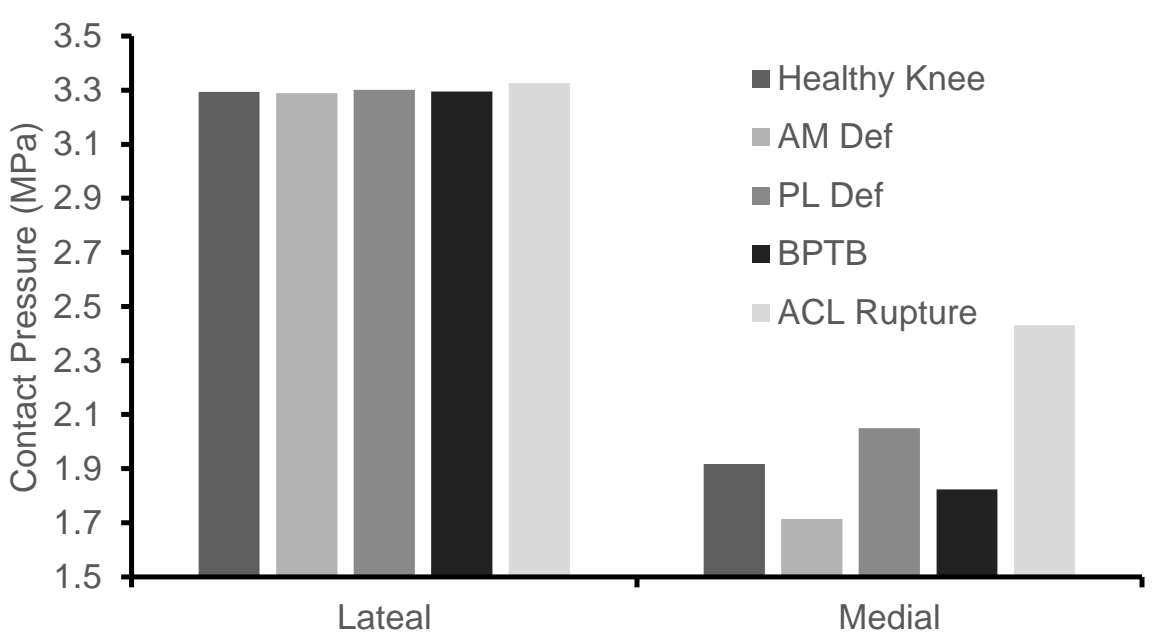

Figure 60: CP of femoral cartilage at $60 \%$ of Gait. 


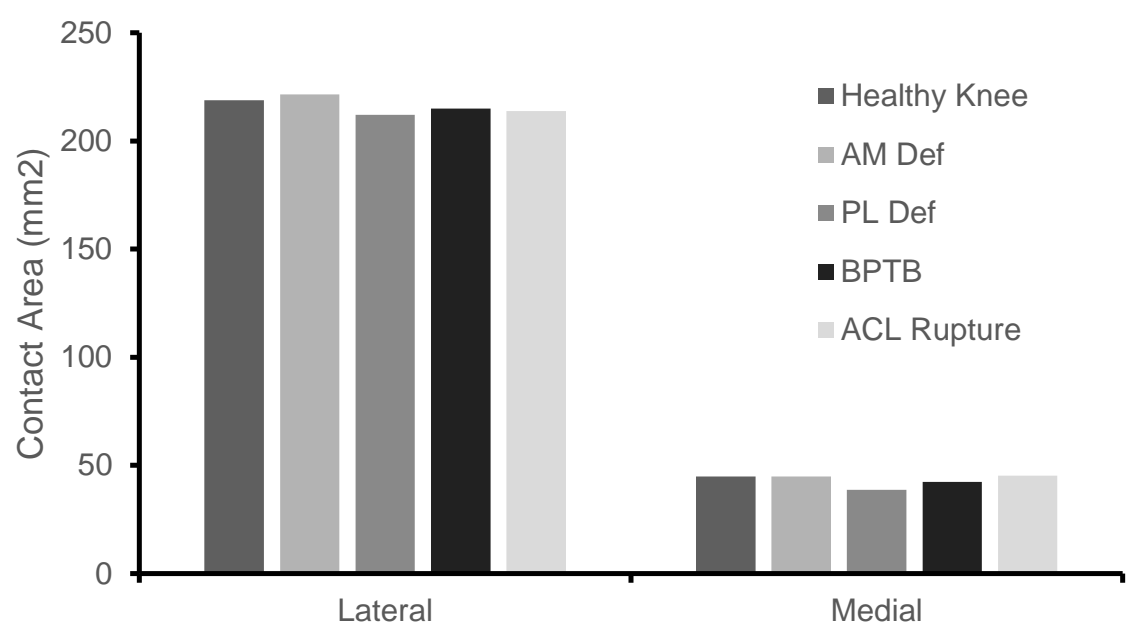

Figure 61 : CA of tibial cartilage at $60 \%$ of Gait.

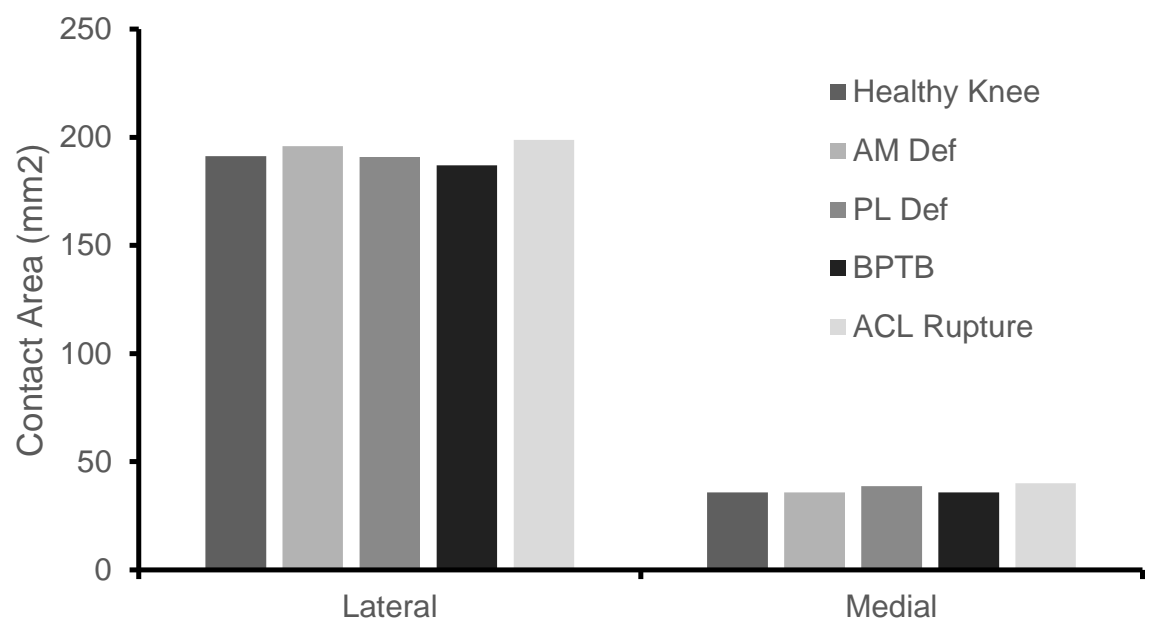

Figure 62: CA of femoral cartilage at $60 \%$ of Gait. 